Pontifícia Universidade Católica $_{\text {a }}$

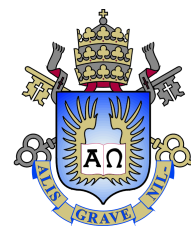

Victoria Menescal Tupper Palhares

\title{
Precoding and Resource Allocation for Cell-Free Massive MIMO Systems
}

Dissertation presented to the Programa de Pós-graduação em Engenharia Elétrica of PUC-Rio in partial fulfillment of the requirements for the degree of Mestre em Engenharia Elétrica.

Advisor: Prof. Rodrigo Caiado de Lamare 


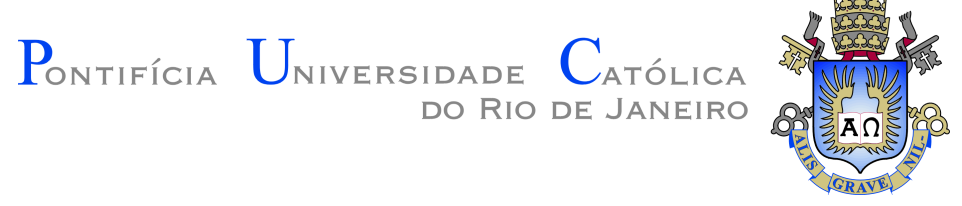

Victoria Menescal Tupper Palhares

Precoding and Resource Allocation for
Cell-Free Massive MIMO Systems

Dissertation presented to the Programa de Pós-graduação em Engenharia Elétrica of PUC-Rio in partial fulfillment of the requirements for the degree of Mestre em Engenharia Elétrica. Approved by the Examination Committee.

Prof. Rodrigo Caiado de Lamare

Advisor

CETUC - PUC-Rio

Prof. Lukas Tobias Nepomuk Landau

CETUC - PUC-Rio

Prof. Marco Antonio Grivet Mattoso Maia

CETUC - PUC-Rio

Prof. Flavio Luiz Duarte Instituto Militar de Engenharia - IME

Rio de Janeiro, August the 21st, 2020 
All rights reserved.

\section{Victoria Menescal Tupper Palhares}

She received her Bachelor of Science in Electrical Engineering degree with emphasis in Electronics \& Computers, from the Pontifical Catholic University of Rio de Janeiro (PUC-Rio), in 2018.

Bibliographic data

Menescal Tupper Palhares, Victoria

Precoding and Resource Allocation for Cell-Free Massive MIMO Systems / Victoria Menescal Tupper Palhares; advisor: Rodrigo Caiado de Lamare. - Rio de Janeiro: PUC-Rio, Departamento de Engenharia Elétrica, 2020.

v., 110 f: il. color. ; $30 \mathrm{~cm}$

Dissertação (mestrado) - Pontifícia Universidade Católica do Rio de Janeiro, Departamento de Engenharia Elétrica.

Inclui bibliografia

1. Engenharia Elétrica - Teses. 2. Sistemas de Múltiplas Antenas Livres de Células;. 3. Pré-codificador MMSE;. 4. Alocação de Potência;. 5. Seleção de PA;. 6. Sistemas de Antenas Distribuídas;. 7. Otimização.. I. Caiado de Lamare, Rodrigo. II. Pontifícia Universidade Católica do Rio de Janeiro. Departamento de Engenharia Elétrica. III. Título. 


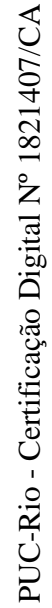

To my family, Cinthia, Carlos Henrique and Alexandra. 


\section{Acknowledgments}

First, I would like to express my endless gratitude to my advisor, Prof. Rodrigo C. de Lamare, for giving me great opportunities and for introducing me to this novel topic. Thanks to him, in these two years, I gained not only academic experience but he has also changed my perspective and attitude towards life. This work wouldn't be possible without his patience, guidance, daily motivation and good influence.

Second, I would like to thank Prof. Lukas T. N. Landau, for his enthusiastic and hard-working contribution to this work. As a teacher and co-author of my papers, he added much in my academic knowledge and has given me an amazing support.

Then, I would like to express my gratitude to André R. Flores, who has a been a true friend and gave great insights in this project. His guidance has given me an incredible support in challenging parts of this work.

I would like to express my love to my family, Cinthia, Carlos Henrique and Alexandra. To my mother, Cinthia, thank you for always teaching me the beauty of knowledge and to never stop learning. She is my true inspiration as an intellectual and a woman. To my father, thank you for showing me how to be moved by passion and for your wisdom. Life is much simpler through his eyes. To Alexandra, my sister and best friend, for always being so caring and being there for me in moments of need.

I would like to thank the other members of my defence committee, Prof. Marco Antonio Grivet and Prof. Flavio Luiz Duarte, for your time, patience and for giving me the honour to present my work.

My sincere thanks to CETUC and the Pontifical Catholic University of Rio de Janeiro (PUC-Rio), with all its professors, students and staff for providing a pleasant environment for me to pursue my studies. The university has been my home for seven and a half years and I will miss everything in it.

Finally, I wish to thank CAPES, CNPq, FAPERJ and PUC-Rio for the financial support.

This study was financed in part by the Coordenação de Aperfeiçoamento de Pessoal de Nível Superior - Brasil (CAPES) - Finance Code 001. 


\section{Abstract}

Menescal Tupper Palhares, Victoria; Caiado de Lamare, Rodrigo (Advisor). Precoding and Resource Allocation for CellFree Massive MIMO Systems. Rio de Janeiro, 2020. 110p. Dissertação de mestrado - Departamento de Engenharia Elétrica, Pontifícia Universidade Católica do Rio de Janeiro.

Cell-Free Massive multiple-input multiple-output (MIMO) systems have emerged in recent years as a combination of massive MIMO, distributed antenna systems (DAS) and network MIMO. This thesis explores the downlink channel of such scenario with single and multiple-antenna access points (APs) and takes into account both perfect and imperfect channel state information (CSI). We propose transmit processing schemes that combine precoding, power allocation and AP selection (APS). To begin with, two APS strategies have been investigated, one based on exhaustive search (ES-APS) and the other on the large-scale fading coefficients (LSAPS), in order to reduce the complexity of cell-free networks. Subsequently, we present two iterative precoding techniques following the minimum meansquare error (MMSE) criterion with total power constraint. The first we call MMSE, with total power constraint. We also incorporate robustness in the developed method, called RMMSE, a robust precoder with total power constraint. As the third element of the proposed schemes, power allocation techniques are developed, with optimal, adaptive and uniform approaches. An optimal power allocation (OPA) algorithm is presented based on the maximization of the minimum signal-to-interference-plus-noise ratio (SINR). The adaptive solution (APA) is characterized by the stochastic gradient of the mean-square error (MSE) and the uniform alternative (UPA) proposes to equalize all power coefficients. All configurations must fulfil an antenna power constraint, imposed by the system. A sum-rate analysis is carried out for all studied techniques and the computational cost of each one is calculated. Numerical results prove that the proposed techniques outperform existing conjugate beamforming (CB) and zero-forcing (ZF) precoders, both with uniform and optimal power allocation, in terms of bit error rate (BER), sum-rate and minimum SINR. Furthermore, we also attest that performance can be maintained or even improved in the presence of APS.

\section{Keywords}

Cell-Free Massive MIMO; MMSE Precoding; Power Allocation; AP Selection; Distributed Antenna Systems; Optimization. 


\section{Resumo}

Menescal Tupper Palhares, Victoria; Caiado de Lamare, Rodrigo. Pré-Codificação e Alocação de Recursos em Sistemas de Múltiplas Antenas Massivos Livres de Células. Rio de Janeiro, 2020. 110p. Dissertação de Mestrado - Departamento de Engenharia Elétrica, Pontifícia Universidade Católica do Rio de Janeiro.

Sistemas de múltiplas antenas livres de células surgiram recentemente como uma combinação de MIMO massivo, sistemas de antenas distribuídas (DAS) e network MIMO. Esta dissertação explora o downlink deste cenário com pontos de acesso (PAs) de uma ou múltiplas antenas e considerando conhecimento perfeito e imperfeito do canal. São desenvolvidos esquemas que combinam pré-codificação, alocação de potência e seleção de PAs (SPA). Para começar, duas estratégias de SPA foram investigadas, uma baseada em busca exaustiva (BE-SPA) e a outra em coeficientes de desvanecimento de larga escala (LE-SPA), com o intuito de reduzir a complexidade das redes livres de células. Subsequentemente, apresentamos duas técnicas iterativas de pré-codificação, todas seguindo o critério Minimum Mean-Square Error (MMSE), combinadas à restrição de potência total. A primeira nós chamamos de MMSE, com restrição de potência total. Nós também incorporamos robustez ao método desenvolvido chamado RMMSE, um pré-codificador robusto com restrição de potência total. Como terceiro elemento da configuração proposta, esquemas de alocação de potência foram desenvolvidos, com abordagens ótimas, adaptativas e uniformes. Um algoritmo de alocação de potência ótima (APO) é apresentado, baseado na maximização da mínima Signal-to-Interference-plus-Noise Ratio (SINR). A solução adaptativa (APA) é caracterizada pelo gradiente estocástico (GE) do mean-square error (MSE) e a alternativa uniforme (UPA) propõe a equalização de todos os coeficientes de potência. Todas as configurações devem respeitar a restrição de potência por antena, imposta pelo sistema. Uma análise de soma das taxas é feita, para todas as técnicas estudadas e o custo computacional de cada uma delas é calculado. Resultados numéricos provam que as técnicas propostas têm performance superior à pré-codificadores Conjugate Beamforming (CB) e Zero-Forcing (ZF), ambos com alocação de potência uniforme e ótima, na forma de taxa de erro de bit (BER), soma das taxas e mínima SINR. Além disso, os resultados atestam que o desempenho pode ser mantido e até melhorado com a aplicação de SPA.

\section{Palavras-chave}


Sistemas de Múltiplas Antenas Livres de Células; Pré-codificador MMSE; Alocação de Potência; Seleção de PA; Sistemas de Antenas Distribuídas; Otimização. 


\section{Table of contents}

1 Introduction $\quad 22$

1.1 Motivation 23

1.2 Contributions 24

1.3 Thesis Outline 25

1.4 Notation 25

$\begin{array}{ll}1.5 & \text { List of Publications }\end{array}$

2 Cell-Free Massive MIMO Systems Review $\quad 27$

2.1 Literature Review 27

2.2 System Model $\quad 32$

2.2.1 Multiple-Antenna APs 33

2.2.2 Large Scale Fading Coefficient Model 33

2.2.3 Channel Estimation 34

2.2.4 Downlink Payload Data Transmission 36

2.3 Precoding and Power Allocation Techniques 37

2.3.1 Conjugate Beamforming 37

2.3.1.1 Optimal Power Allocation (OPA) 38

2.3.1.2 Uniform Power Allocation (UPA) 39

2.3.2 Zero Forcing 40

2.3.2.1 Optimal Power Allocation (OPA) 41

2.3.2.2 Uniform Power Allocation (UPA) 41

2.4 Transmit Wiener Filter for MIMO Systems 42

2.5 Access Point Selection (APS) 42

2.5.1 Received-Power-Based Selection 42

2.5.2 Largest-Large-Scale-Fading-Based Selection 43

2.6 Numerical Results 44

2.7 Summary 45

3 Iterative AP Selection, MMSE Precoding and Power Allocation for CellFree Massive MIMO Systems $\quad 47$

3.1 Access Point Selection (APS) $\quad 47$

$\begin{array}{lll}3.1 .1 & \text { Precoding with APS } & 47\end{array}$

3.1.2 Exhaustive Search Selection (ES-APS) 49

3.1.3 Large-Scale-Fading-Based Selection with Fixed Number of APs (LSAPS) 49

3.2 Iterative MMSE Precoder with Total Power Constraint 50

3.3 Power Allocation $\quad 55$

3.3.1 Optimal Power Allocation (OPA) 55

3.3.2 Adaptive Power Allocation (APA) 56

$\begin{array}{ll}\text { 3.3.3 Uniform Power Allocation (UPA) } & 58\end{array}$

$\begin{array}{ll}3.4 \text { Analysis } & 58\end{array}$

$\begin{array}{lll}3.4 .1 & \text { Sum-Rate } & 59\end{array}$

3.4.2 Computational Complexity 61

3.5 Numerical Results $\quad 62$ 
4 Iterative Robust MMSE Precoding and Power Allocation for Cell-Free Massive MIMO Systems

4.1 Iterative RMMSE Precoder with Total Power Constraint $\quad 70$

4.2 Robust Power Allocation $\quad 75$

$\begin{array}{lll}\text { 4.2.1 Optimal Power Allocation (OPA) } & 75\end{array}$

$\begin{array}{ll}\text { 4.2.2 Uniform Power Allocation (UPA) } & 76\end{array}$

$\begin{array}{lll}4.3 \text { Analysis } & 77\end{array}$

$\begin{array}{lll}\text { 4.3.1 Sum-Rate } & 77\end{array}$

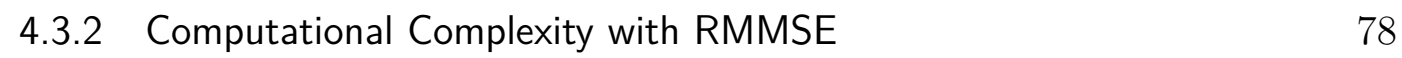

$\begin{array}{lll}4.4 & \text { Numerical Results } & 79\end{array}$

$\begin{array}{lll}4.5 & \text { Summary } & 87\end{array}$

$\begin{array}{lll}5 & \text { Conclusion and Future Work } & 88\end{array}$

$\begin{array}{lr}\text { Bibliography } & 90\end{array}$

$\begin{array}{lll}\text { A Computational Complexity } & 97\end{array}$

$\begin{array}{lll}\text { A.1 } & \text { APS Complexity } & 97\end{array}$

$\begin{array}{lll}\text { A.2 } & \text { Precoders } & 97\end{array}$

A.3 Precoders + Power Allocation 101

$\begin{array}{lll}\text { A.4 SINR Computation } & 102\end{array}$

$\begin{array}{lll}\text { A.5 Power Allocation } & 105\end{array}$ 


\section{List of figures}

Figure 2.1 Cell-Free Massive MIMO System. 32

Figure 2.2 TDD Protocol 35

Figure 2.3 Sum-Rate vs. SNR with $L=128, \mathrm{~N}=1, K=16, n=1$, and 120 channel realizations.

Figure 2.4 Sum-Rate vs. SNR with $L=128, \mathrm{~N}=1, K=16$, $n=0.99$ and 120 channel realizations

Figure 3.1 MSE vs. Iterations with $L=24, N=4, S=12, K=8$, $n=1, \mathrm{SNR}=25 \mathrm{~dB}, \mu=0.25$ and $E_{t r}=M \rho_{f}$.

Figure 3.2 Sum-Rate vs. SNR with $L=5, \mathrm{~N}=1, S=3, K=2$, $n=0.99,120$ channel realizations and $E_{t r}=M \rho_{f}$.

Figure 3.3 Sum-Rate vs. SNR with $L=5, \mathrm{~N}=1, S=3, K=2$, $n=0.99,120$ channel realizations and $E_{t r}=M \rho_{f}$.

Figure 3.4 Minimum SINR vs. SNR with $L=128, \mathrm{~N}=1, S=64$, $K=16, n=0.99,120$ channel realizations and $E_{t r}=M \rho_{f}$.

Figure 3.5 Minimum SINR vs. SNR with $L=128, \mathrm{~N}=1, S=64$, $K=16, n=0.99,120$ channel realizations and $E_{t r}=M \rho_{f}$.

Figure 3.6 Sum-Rate vs. SNR with $L=128, \mathrm{~N}=1, S=64, K=16$, $n=0.99,120$ channel realizations and $E_{t r}=M \rho_{f}$.

Figure 3.7 Sum-Rate vs. SNR with $L=128, \mathrm{~N}=1, S=64, K=16$, $n=0.99,120$ channel realizations and $E_{t r}=M \rho_{f}$.

Figure 3.8 BER vs. SNR with $L=24, N=4, S=12, K=8$, $n=1,120$ channel realizations, 100 symbols per packet and $E_{t r}=M \rho_{f}$.

Figure 3.9 BER vs. SNR with $L=24, N=4, S=12, K=8$, $n=1,120$ channel realizations, 100 symbols per packet and $E_{t r}=M \rho_{f}$.

Figure 3.10 BER vs. SNR with $L=24, N=4, K=8, n=1,120$ channel realizations, 100 symbols per packet and $E_{t r}=M \rho_{f}$.

Figure 4.1 BER vs. SNR with $L=96, N=1, K=8, n=0.99$,

120 channel realizations, 100 symbols per packet and $E_{t r}=M \rho_{f} .80$

Figure 4.2 BER vs. SNR with $L=24, N=4, K=8, n=0.99$, 120 channel realizations, 100 symbols per packet and $E_{t r}=M \rho_{f} .81$

Figure 4.3 BER vs. SNR with $L=96, N=1, K=8, n=0.99$, 120 channel realizations, 100 symbols per packet and $E_{t r}=M \rho_{f} .82$

Figure 4.4 BER vs. SNR with $L=24, N=4, K=8, n=0.99$, 120 channel realizations, 100 symbols per packet and $E_{t r}=M \rho_{f} .82$

Figure 4.5 BER vs. SNR with $L=96, N=1, S=48, K=8$, $n=0.99,120$ channel realizations, 100 symbols per packet and $E_{t r}=M \rho_{f}$.

Figure 4.6 BER vs. SNR with $L=24, N=4, S=12, K=8$, $n=0.99,120$ channel realizations, 100 symbols per packet and $E_{t r}=M \rho_{f}$. 
Figure 4.7 Sum-Rate vs. SNR with $L=128, \mathrm{~N}=1, K=16, n=0.9$, 120 channel realizations and $E_{t r}=M \rho_{f}$.

Figure 4.8 Sum-Rate vs. SNR with $L=128, \mathrm{~N}=1, K=16, n=0.9$, 120 channel realizations and $E_{t r}=M \rho_{f}$.

Figure 4.9 Sum-Rate vs. SNR with $L=128, N=1, S=64, K=16$, $n=0.9,120$ channel realizations and $E_{t r}=M \rho_{f}$.

Figure 4.10 Minimum SINR vs. SNR with $L=128, \mathrm{~N}=1, S=64$, $K=16, n=0.9,120$ channel realizations and $E_{t r}=M \rho_{f}$. 


\section{List of tables}

$\begin{array}{lll}\text { Table 3.1 Computational Complexity } & 61\end{array}$

Table 4.1 Computational Complexity with RMMSE 79 


\section{List of Algorithms}

$1 \quad$ Bisection Method [1] 38

2 Received-Power-Based Selection [2] 43

3 Largest-Large-Scale-Fading Based Selection [2] 44

4 Large-Scale-Fading-Based Selection with Fixed Number of APs (LS-APS) 50

5 Adaptive Power Allocation Algorithm Based on the Stochastic Gradient (APA) 57

6 Iterative RMMSE Precoding With Total Power Constraint (RMMSE) 


\section{List of Abbreviations}

$4 \mathrm{G}$ - Fourth Generation

$5 \mathrm{G}$ - Fifth Generation

APA - Adaptive Power Allocation

AP - Access Point

APS - Access Point Selection

BS - Base Station

BER - Bit Error Rate

CAPES - Coordenação de Aperfeiçoamento de Pessoal de Nível Superior

CAS - Co-located Antenna Systems

CB - Conjugate Beamforming

CDI - Channel Direction Information

CETUC - Center for Telecommunications Studies

CNPq - Conselho Nacional de Desenvolvimento Científico e Tecnológico

CoMP - Coordinated Multi-Point Joint Processing

CPU - Central Processing Unit

CSI - Channel State Information

DAS - Distributed Antenna Systems

DL - Diagonal Loading

EE - Energy Efficiency

ES - Exhaustive Search

ES-APS - Exhaustive Search Selection

FAPERJ - Fundação Carlos Chagas Filho de Amparo à Pesquisa do Estado

do Rio de Janeiro

IPM - Interior-Point Methods

L-DAS - Large-Scale Distributed Antenna Systems

LS - Large-Scale Fading

LS-APS - Large-Scale-Fading-Based Selection with Fixed Number of APs

LSFD - Large-Scale Fading Decoding

M2M - Machine-to-Machine

MF - Matched Filter

MIMO - Multiple-Input Multiple-Output

MMSE - Minimum Mean-Square Error

mmWave - Millimeter Wave 
MSE - Mean-Square Error

NS - Non Selective Scheme

OPA - Optimal Power Allocation

QoS - Quality of Service

QPSK - Quadrature Phase Shift Keying

RMMSE - Robust MMSE Precoder

RRH - Remote Radio Head

RS - Relay Station

RV - Random Variable

SDP - Semidefinite Programming

SE - Spectral Efficiency

SG - Stochastic Gradient

SINR - Signal-to-interference-plus-noise ratio

SNR - Signal-to-noise ratio

SOCP - Second-Order Cone Program

TDD - Time Division Duplex

THP - Tomlinson-Harashima Precoder

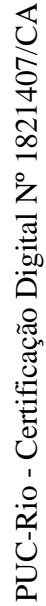

UPA - Uniform Power Allocation

WF - Wiener Filter

ZF - Zero-Forcing 


\section{List of Symbols}

$L$ - number of APs

$N$ - number of antenna elements per AP

$M$ - total number of antenna elements

$K$ - number of users

$g_{m, k}$ - channel coefficient between the $m$ th antenna element and the $k$ th user, where $g_{m, k} \in \mathbb{C}^{1 \times 1}$

$\hat{g}_{m, k}$ - channel estimation coefficient between the $m$ th antenna element and the $k$ th user, where $\hat{g}_{m, k} \in \mathbb{C}^{1 \times 1}$

$\tilde{g}_{m, k}$ - channel estimation error coefficient between the $m$ th antenna element and the $k$ th user, where $\tilde{g}_{m, k} \in \mathbb{C}^{1 \times 1}$

$\mathbf{g}_{k}$ - channel vector for user $k$, where $\mathbf{g}_{k} \in \mathbb{C}^{M \times 1}$

$\hat{\mathbf{g}}_{k}$ - channel estimation vector for user $k$, where $\hat{\mathbf{g}}_{k} \in \mathbb{C}^{M \times 1}$

$\tilde{\mathbf{g}}_{k}$ - channel estimation error vector for user $k$, where $\tilde{\mathbf{g}}_{k} \in \mathbb{C}^{M \times 1}$

$\hat{\mathbf{g}}_{m}$ - channel estimation vector for the $m$ th antenna, where $\hat{\mathbf{g}}_{m} \in \mathbb{C}^{1 \times K}$

$\mathbf{G}$ - channel matrix, where $\mathbf{G} \in \mathbb{C}^{M \times K}$

$\hat{\mathbf{G}}$ - channel estimation matrix, where $\hat{\mathbf{G}} \in \mathbb{C}^{M \times K}$

$\tilde{\mathbf{G}}$ - channel estimation error matrix, where $\tilde{\mathbf{G}} \in \mathbb{C}^{M \times K}$

$\boldsymbol{\beta}$ - large-scale fading matrix, where $\boldsymbol{\beta} \in \mathbb{R}^{M \times K}$

$\beta_{m, k}$ - large-scale fading coefficient between the $m$ th antenna element and the $k$ th user, where $\beta_{m, k} \in \mathbb{R}^{1 \times 1}$

$\boldsymbol{\beta}_{k}$ - large-scale fading vector associated to the $k$ th user, where $\boldsymbol{\beta}_{k} \in \mathbb{R}^{M \times 1}$

$h_{m, k}$ - small-scale fading coefficient between the $m$ th antenna element and the $k$ th user, where $h_{m, k} \in \mathbb{C}^{1 \times 1}$

$\mathrm{PL}_{m, k}$ - path loss coefficient between the $m$ th antenna element and the $k$ th user, where $\mathrm{PL}_{m, k} \in \mathbb{R}^{1 \times 1}$

$\sigma_{s h}$ - shadow fading standard deviation

$z_{m, k}$ - shadowing coefficient, where $z_{m, k} \in \mathbb{R}^{1 \times 1}$

$d_{m, k}$ - distance between the $m$ th antenna element and the $k$ th user

$f_{\text {freq }}$ - carrier frequency in $\mathrm{MHz}$

$h_{\mathrm{AP}}-\mathrm{AP}$ antenna height in meters

$h_{\mathrm{u}}$ - user antenna height in meters

$\tau$ - length of pilot sequences

$\boldsymbol{\Pi}_{k}$ - transmit pilot sequence by the $k$ th user, where $\boldsymbol{\Pi}_{k} \in \mathbb{C}^{\tau \times 1}$ 
$\mathbf{y}_{m}$ - received signal sequence by the $m$ th antenna in the training step, where

$\mathbf{y}_{m} \in \mathbb{C}^{\tau \times 1}$

$\rho_{r}$ - uplink power

$\mathbf{w}_{m}$ - is the additive noise received by the $m$ th user, where $\mathbf{w}_{m} \in \mathbb{C}^{\tau \times 1}$

$\boldsymbol{\alpha}$ - matrix of variances of $\hat{g}_{m, k}$, for $m=1, \ldots, M$ and $k=1, \ldots, K$, where

$\boldsymbol{\alpha} \in \mathbb{R}^{M \times K}$

$\alpha_{m, k}$ - variance of $\hat{g}_{m, k}$, where $\alpha_{m, k} \in \mathbb{R}^{1 \times 1}$

$n$ - ratio between $\alpha_{m, k}$ and $\beta_{m, k}$

$y_{k}$ - signal received by the $k$ th user, where $y_{k} \in \mathbb{C}^{1 \times 1}$

$\mathbf{y}$ - vector combining the signal received by all users, where $\mathbf{y} \in \mathbb{C}^{K \times 1}$

$\rho_{f}$ - maximum transmitted power of each antenna

$\mathbf{P}$ - generic precoding matrix, where $\mathbf{P} \in \mathbb{C}^{M \times K}$

$\mathbf{s}$ - zero mean symbol vector, where $\mathbf{s} \in \mathbb{C}^{K \times 1}$

$s_{k}$ - data symbol intended for user $k$, where $s_{k} \in \mathbb{C}^{1 \times 1}$

$\sigma_{s}^{2}$ - symbol variance

$\mathbf{C}_{s}$ - symbol covariance matrix, $\mathbf{C}_{s} \in \mathbb{R}^{K \times K}$

$w_{k}$ - additive noise for user $k$, where $w_{k} \in \mathbb{C}^{1 \times 1}$

$\mathbf{w}$ - additive noise vector, where $\mathbf{w} \in \mathbb{C}^{K \times 1}$

$\sigma_{w}^{2}$ - noise variance

SNR - signal-to-noise ratio

$\mathbf{C}_{w}$ - noise covariance matrix, $\mathbf{C}_{w} \in \mathbb{R}^{K \times K}$

$T_{0}$ - noise temperature in Kelvin

$k_{B}$ - Boltzmann constant in Joule per Kelvin

$B$ - bandwidth in $\mathrm{Hz}$

$\mathrm{NF}$ - noise figure in Watts

$R_{\mathrm{k}, \mathrm{CB}}$ - achievable rate of user $k$ with $\mathrm{CB}$ precoding

$R_{\mathrm{CB}}$ - sum-rate with $\mathrm{CB}$ precoding

SINR $_{\mathrm{k}, \mathrm{CB}}-\mathrm{SINR}$ of user $k$ with CB precoding

$A_{i}$ - parameter of the SOCP, where $A_{i} \in \mathbb{R}^{(M * K)+1 \times(M * K)+1}$

$c_{i}$ - parameter of the SOCP, where $c_{i} \in \mathbb{R}^{(M * K)+1 \times 1}$

$b_{i}$ - parameter of the SOCP, where $b_{i} \in \mathbb{R}^{(M * K)+1 \times 1}$

$d_{i}$ - parameter of the SOCP, where $d_{i} \in \mathbb{R}^{1 \times 1}$

$\beta_{m}^{\max }$ - largest coefficient in vector $\boldsymbol{\beta}_{m}$, where $\beta_{m}^{\max } \in \mathbb{R}^{1 \times 1}$

$\kappa$ - coefficient for (2-29), where $\kappa \in \mathbb{R}^{1 \times 1}$

$R_{\mathrm{k}, \mathrm{ZF}}$ - achievable rate of user $k$ with ZF precoding

$R_{\mathrm{ZF}}$ - sum-rate with ZF precoding

$\mathrm{SINR}_{\mathrm{k}, \mathrm{ZF}}$ - SINR of user $k$ with ZF precoding

- auxiliary vector related to the desired signal of all users, where $\boldsymbol{\psi} \in \mathbb{C}^{K \times 1}$

$k$ - the $k$ th element of vector $\boldsymbol{\psi}$, where ${ }_{k} \in \mathbb{C}^{1 \times 1}$ 
$\phi_{k}$ - auxiliary vector related to the interference in user $k$ created by all other users, where $\phi_{k} \in \mathbb{C}^{(K-1) \times 1}$

$\phi_{k, i}$ is the $i$ th element of vector $\phi_{k}$, where $\phi_{k, i} \in \mathbb{C}^{1 \times 1}$

$\gamma_{k}$ - auxiliary vector related to the CSI error, where $\gamma_{k} \in \mathbb{C}^{K \times 1}$

$\gamma_{k, i}$ - the $i$ th element of vector $\gamma_{k}$, where $\gamma_{k, i} \in \mathbb{C}^{1 \times 1}$

$\boldsymbol{\delta}_{m}$ - auxiliary vector related to the precoder energy, where $\boldsymbol{\delta}_{m} \in \mathbb{C}^{K \times 1}$

$\delta_{m, i}$ - the $i$ th element of vector $\boldsymbol{\delta}_{m}$, where $\delta_{m, i} \in \mathbb{C}^{1 \times 1}$

$\mathbf{P}_{\mathrm{WF}}$ - Transmit WF, where $\mathbf{P}_{\mathrm{WF}} \in \mathbb{C}^{M \times K}$

$f_{\mathrm{WF}}$ - final normalization factor of the Transmit WF, where $f_{\mathrm{WF}} \in \mathbb{R}_{+}^{1 \times 1}$

$\mathbf{E}$ - receive filter in (2-39), where $\mathbf{E} \in \mathbb{C}^{K \times K}$

$\mathbf{F}$ - auxiliary matrix from (2-43), where $\mathbf{F} \in \mathbb{C}^{M \times M}$

$\mathrm{DS}_{k}$ - total received power of the desired signal of the $k$ th user

$\chi(l, k)$ - contribution of the $l$ th $\mathrm{AP}$ in to the total received power of the $k$ th user

$\zeta$ - tolerance of Algorithms 2 and 3.

$\mathcal{A}_{k}$ - set of APs selected by user $k$ in Algorithm 2.

$\mathcal{U}_{l}$ - set of users served by the $l$ th AP in Algorithms 2 and 3.

$L_{0, k}$ - set of APs selected by user $k$ in Algorithm 3.

$\bar{\beta}_{l, k}$ - element of the sorted (in descending order) set of the set $\left\{\beta_{1, k}, \ldots, \beta_{L, k}\right\}$

$S$ - number of selected APs

Q - antenna selection matrix, where $\mathbf{Q} \in \mathbb{N}^{M \times K}$

$q_{m, k}$ - antenna selection coefficient, where $q_{m, k} \in\{0,1\}$

$\mathbf{q}_{k}$ - antenna selection vector associated to user $k$, where $\mathbf{q}_{k} \in \mathbb{N}^{M \times 1}$

$\mathbf{V}$ - matrix with all possible combinations of APS, where $\mathbf{V} \in \mathbb{N}^{L \times\left(\begin{array}{l}L \\ S\end{array}\right)}$

$\mathrm{SINR}_{k}$ - signal-to-interference-plus-noise ratio of user $k$

$A_{1}$ - desired signal by user $k$

$A_{2, i}$ - interference caused by user $i$ in user $k$

$A_{3}$ - channel estimation error of user $k$

$\mathbf{N}$ - power allocation matrix, where $\mathbf{N} \in \mathbb{R}_{+}^{K \times K}$

$\eta_{m, k}$ - power coefficient used by the $m$ th antenna for transmission to user $k$, where $\eta_{m, k} \in \mathbb{R}_{+}^{1 \times 1}$

$\eta_{m, k}^{\prime \prime}$ - power coefficient used by the $m$ th antenna for transmission to user $k$, where $\eta_{m, k}^{\prime \prime}=\sqrt{\eta_{m, k}}$

$\eta_{m}$ - power coefficient of the $m$ th antenna, where $\eta_{m} \in \mathbb{R}_{+}^{1 \times 1}$

$\eta_{k}$ - power coefficient of user $k$, where $\eta_{k} \in \mathbb{R}_{+}^{1 \times 1}$

$\boldsymbol{\eta}$ - power coefficient vector, where $\boldsymbol{\eta} \in \mathbb{R}_{+}^{K \times 1}$

$f$ - initial normalization factor of the MMSE precoder, where $f \in \mathbb{R}_{+}^{1 \times 1}$

$\mathbf{x}$ - transmitted signal, where $\mathbf{x} \in \mathbb{C}^{M \times 1}$

$x_{m}$ - transmitted signal by the $m$ th antenna element, where $x_{m} \in \mathbb{C}^{1 \times 1}$ 
$E_{t r}$ - total transmitted power

$\lambda$ - Lagrange multiplier

$\Gamma$ - Lagrange multiplier

A - MMSE precoder combined with power allocation, where $\mathbf{A} \in \mathbb{C}^{M \times K}$

$\mathbf{a}_{m}$ - the $m$ th row of matrix $\mathbf{A}$, where $\mathbf{a}_{m} \in \mathbb{C}^{1 \times K}$

$\mathbf{P}_{\mathrm{ZF}}$ - ZF precoder with power allocation, where $\mathbf{P}_{\mathrm{ZF}} \in \mathbb{C}^{M \times K}$

$\mathbf{P}_{\mathrm{MMSE}}$ - MMSE precoder with total power constraint, where $\mathbf{P}_{\mathrm{MMSE}} \in \mathbb{C}^{M \times K}$

$f_{\mathrm{MMSE}}$ - final normalization factor of the MMSE precoder with total power constraint, where $f_{\mathrm{MMSE}} \in \mathbb{R}_{+}^{1 \times 1}$

$\mathbf{I}_{D}$ - identity matrix of size $D \times D$

$\mathbf{0}_{D \times J}$ - zero matrix with dimensions $D \times J$

$\mathbf{N}_{\text {MMSE }}$ - final power allocation matrix for the MMSE precoder with total power constraint, where $\mathbf{N}_{\mathrm{MMSE}} \in \mathbb{R}_{+}^{K \times K}$

$R_{\mathrm{k}, \mathrm{MMSE}}$ - achievable rate of user $k$ with MMSE precoding with total power constraint

$R_{\mathrm{MMSE}}$ - sum-rate with MMSE precoding with total power constraint

SINR $_{\mathrm{k}, \mathrm{MMSE}}$ - SINR of user $k$ with MMSE precoding with total power constraint

$\mathbf{P}_{\text {RMMSE }}$ - RMMSE precoder with total power constraint, where $\mathbf{P}_{\text {RMMSE }} \in$ $\mathbb{C}^{M \times K}$

$f_{\text {RMMSE }}$ - final normalization factor of the RMMSE precoder with total power constraint, where $f_{\text {RMMSE }} \in \mathbb{R}_{+}^{1 \times 1}$

$\mathbf{H}$ - auxiliary diagonal matrix part of $\mathbf{P}_{\text {RMMSE }}$, where $\mathbf{H} \in \mathbb{C}^{M \times M}$

$\mathbf{N}_{\text {RMMSE }}$ - final power allocation matrix for the RMMSE precoder with total power constraint, where $\mathbf{N}_{\mathrm{RMMSE}} \in \mathbb{R}_{+}^{K \times K}$

$R_{\mathrm{k}, \mathrm{RMMSE}}$ - achievable rate of user $k$ with RMMSE precoding with total power constraint

$R_{\text {RMMSE }}$ - sum-rate with RMMSE precoding with total power constraint

SINR $_{\mathrm{k}, \mathrm{RMMSE}}$ - SINR of user $k$ with RMMSE precoding with total power constraint

$\mathbf{p}_{m}-m$ th row of precoder $\mathbf{P}_{\mathrm{MMSE}}$ or $\mathbf{P}_{\mathrm{RMMSE}}$, where $\mathbf{p}_{m} \in \mathbb{C}^{1 \times K}$

$\mathbf{p}_{k}-k$ th column of precoder $\mathbf{P}_{\mathrm{MMSE}}$ or $\mathbf{P}_{\mathrm{RMMSE}}$, where $\mathbf{p}_{k} \in \mathbb{C}^{M \times 1}$

$t$ - midpoint of the interval $\left(t_{b}, t_{e}\right)$

$t_{b}$ - lower limit of the bisection method

$t_{e}$ - upper limit of the bisection method

$T_{\mathrm{OPA}}$ - number of iterations of the OPA algorithm

$\mu$ - step size of the APA algorithm

$T_{\mathrm{APA}}$ - number of iterations of the APA algorithm

$(\cdot)^{*}$ - complex conjugate 
$(\cdot)^{T}-$ transpose

$(\cdot)^{H}$ - Hermitian operator

$(\cdot)^{\prime}$ - variable after APS

$\mathbb{E}[\cdot]$ - expected value

$\operatorname{tr}(\cdot)$ - trace of a matrix

$\operatorname{Re}(\cdot)$ - real part of the argument

$\|\cdot\|_{2}$ - Euclidean norm

$\|\cdot\|_{F}$ - Frobenius norm

$\odot$ - Hadamard or element-wise product

$\operatorname{diag}\{\mathbf{D}\}$ - retains the main diagonal elements of a generic matrix $\mathbf{D}$ in a column vector

$x \sim \mathcal{N}\left(0, \sigma^{2}\right)$ - Gaussian random variable $(\mathrm{RV}) x$ with zero mean and variance $\sigma^{2}$

$x \sim \mathcal{C N}\left(0, \sigma^{2}\right)$ - circularly symmetric complex Gaussian RV $x$ with zero mean and variance $\sigma^{2}$

$\left(\begin{array}{l}a \\ b\end{array}\right)$ - Combination of $a$ elements in $b$ elements. 


\section{1 \\ Introduction}

Massive multiple-input multiple-output (MIMO) systems have been extensively investigated as a very attractive solution to the fifth generation (5G) of cellular networks. By considering a base station (BS) with many antennas serving simultaneously many users in the same time-frequency resource, this technology can provide high throughput, energy efficiency (EE) and reliability, with simple signal processing [3].

In the context of massive MIMO systems and their potential deployments, possible setups include those with co-located antenna systems (CAS) and distributed antenna systems (DAS). In a CAS, a BS is co-located at the center of each cell, with the advantage of low backhaul requirement. Conversely, in DAS, remote radio heads (RRHs) are spread all over the cell and connected to a central processing unit (CPU) at a BS by optical fiber, coaxial cable or microwave [4]. The latter has been examined over the last decade thanks to its open architecture, flexible resource management, higher power efficiency and larger capacities than CAS, due to the shorter distances between RRHs and users and exploitation of diversity against shadow fading, [5].

In MIMO technology, network MIMO has been suggested in the interest of mitigating the interference between cells. By providing cooperation between $\mathrm{BSs}$, users receive their desired signals through the ones close to them [6]. This has been proven to improve spectral efficiency (SE) and overall system performance [7], at the cost of higher complexity, due to a great amount of data being shared between BSs, CPU and users.

A special case of a large DAS with cooperative BSs has been proposed in [8], where randomly-located single antenna access points (APs) serve simultaneously a much smaller number of users. All APs cooperate phase-coherently via a backhaul network, and serve all users in the same time-frequency resource via time-division duplex (TDD) operation. Unlike Multi-cell Massive MIMO, in the described structure, called Cell-Free Massive MIMO, there are no cell boundaries and consequently, no interference between cells.

In cell-free systems, all APs are connected to a CPU in order to process payload data and to perform power control. Due to the fact that in these setups, APs do not have perfect channel state information (CSI), each one performs 
channel estimation through uplink pilots without sharing information with the CPU. The channel estimates are used to perform precoding on the downlink and detection on the uplink.

The great aspect of these systems is their computational simplicity concerning signal processing and the exploitation of favorable propagation and channel hardening. These phenomena enable the use of computationally efficient and globally optimal algorithms for power control and simple pilot assignment.

\section{1}

\section{Motivation}

Although many works have studied massive MIMO, cell-free systems are quite recent. Precoding schemes with power allocation applied to this type of network have been introduced in $[8,9]$. In the studies in $[8,9]$, conjugate beamforming ( $\mathrm{CB}$ ) and zero-forcing ( $\mathrm{ZF}$ ) precoders have been indicated, with optimal and uniform power allocations, involving convex optimization. In [9], four solutions for the CB precoder are provided, considering quasiconvex and heuristic approaches. For the ZF precoder, two schemes are given, one solving a quasilinear problem and another with a low complexity solution.

Despite the fact that many works have employed minimum mean-square error (MMSE) based precoders in MIMO systems, the technology has never been developed and applied to cell-free networks before. In [10], a MMSE processing is examined for the uplink. Here, we devise MMSE solutions for the downlink, based on different constraints required by the system.

Naturally, as it was previously derived based on the ZF precoder in [9], for the MMSE precoder it was necessary as well to derive appropriate power allocation techniques. For this matter, not only we provide the standard solution with a max-min problem based on the MMSE precoder, but also an adaptive one using a stochastic gradient (SG) algorithm, with lower computational complexity.

To address the increasing backhaul requirements necessary for cell-free networks, AP selection (APS) has been suggested. In [2], two selection schemes have been investigated. Motivated by them, we decided to analyse the optimal selection, based on an exhaustive search (ES), and to propose a suboptimal one to approach the performance of the former.

Robust techniques have been extensively applied to sensor arrays and MIMO systems and have the potential to mitigate the effect of imperfect CSI in cell-free networks. Since the robustness of cell-free systems has never been approached previously in the literature, in this work, we examine this issue. 
Based on previous robust MMSE precoders applied to MIMO systems, [11-15], we develop one of our own, fulfilling cell-free systems requirements.

\section{2}

\section{Contributions}

In summary, the main contributions of this work are:

- Optimal and suboptimal APS schemes are presented. The first is designed through ES, but requires high computational complexity. As an alternative, a suboptimal technique, with lower complexity, based on the large-scale fading (LS) coefficients is developed, with the objective of approximating the optimal solution.

- An iterative linear MMSE precoder with total power constraint is formulated, which takes into account the power allocation matrix in its derivation, unlike existing approaches.

- An iterative robust MMSE precoder with total power constraint (RMMSE) is derived, taking into account the power allocation matrix in its derivation, with the purpose of mitigating the effects of channel estimation errors.

- Optimal and uniform power allocation techniques are introduced, aiming to maximize the minimum signal-to-interference-plus-noise ratio (SINR), with per-antenna power constraint. An adaptive power allocation (APA) algorithm is also presented. Nevertheless, in this case, it is based on the minimization of the mean-square error (MSE), with an antenna power constraint as well.

- Analysis of the achievable rate expressions of the studied methods are obtained, as a means to enable optimal power allocation (OPA).

- An analysis of the computational complexity of the techniques in terms of arithmetic operations is carried out.

- Numerical results are shown, in terms of sum-rate, minimum SINR and bit error rate (BER), to provide insight on the potential of the proposed schemes. 


\section{3}

\section{Thesis Outline}

This thesis is organized as follows:

- In Chapter 2, a review of the most important works in massive MIMO, DAS and cooperative networks is given. Then, we introduce the state of the art of cell-free networks and give a short summary of robust techniques. After that, we present the system model, channel estimation and downlink transmission. In the end of the chapter, we present the latest precoding, power allocation and APS techniques previously designed in the literature.

- In Chapter 3, we present two techniques for APS, followed by an MMSE precoder designed with total power constraint. Next, we introduce three power allocation techniques, with optimal, adaptive and uniform strategies. A sum-rate analysis is carried out and the computational cost of the techniques is calculated. Numerical results and discussions are then displayed in the end of the chapter.

- In Chapter 4, a robust MMSE (RMMSE) precoder is presented, with total power constraint. Then, a power allocation scheme based on the RMMSE precoder is proposed. A sum-rate analysis based on the methods is developed, and the computational cost, calculated. In the end, numerical results and discussions are carried out.

- In Chapter 5, conclusions and future challenges of this work are drawn.

\section{4}

\section{Notation}

See List of Symbols on pages 17-21.

\section{5}

\section{List of Publications}

Some results of this dissertation are under review in the following conferences and journals:

- Victoria M. T. Palhares, Rodrigo C. de Lamare, Andre R. Flores and Lukas T. N. Landau, "Seleção de Pontos de Acesso, Pré-Codificação MMSE e Alocação de Potência Iterativa para Sistemas de Múltiplas Antenas Livres de Células", XXXVIII Simpósio Brasileiro de Telecomunicações e Processamento de Sinais (SBrT), 2020, submitted.

Journal Papers: 
- Victoria M. T. Palhares, Rodrigo C. de Lamare, Andre R. Flores and Lukas T. N. Landau, "Iterative AP Selection, MMSE Precoding and Power Allocation in Cell-Free Massive MIMO Systems", IET Communications, 2020, submitted.

- Victoria M. T. Palhares, Andre R. Flores and Rodrigo C. de Lamare, "Robust MMSE Precoding and Power Allocation for Cell-Free Massive MIMO Systems", IEEE Transactions on Vehicular Technology, 2020, submitted. 


\section{2}

\section{Cell-Free Massive MIMO Systems Review}

In this chapter, we review the literature on massive MIMO, DAS and cooperative networks that have lead to the development of Cell-Free Massive MIMO Systems. Subsequently, a review of the main contributions in cell-free networks is given. Next, we provide an overview of robust techniques and their benefits, when applied to MIMO systems.

The second part of this chapter presents a description of the cell-free system model, channel estimation and transmission of downlink payload data. Precoding, power allocation and APS techniques that were previously derived are presented. Numerical results are showcased at the end of the chapter to provide an insight on the discussed strategies.

\section{1}

\section{Literature Review}

In the past decade, many works have discussed what the $5 \mathrm{G}$ of wireless communications could be. Instead of an incremental advance compared to the fourth generation $(4 \mathrm{G}), 5 \mathrm{G}$ is actually a fresh new start in the telecommunications area, rethinking network architecture, signal processing, efficiency and coverage, with the purpose of delivering high rates for all users [16].

Among the many technologies that have been researched so as to make this generation possible, the literature has been filled with subjects such as millimetre wave (mmWave), device-centric architectures, machine-to-machine (M2M) communications and massive MIMO.

Massive MIMO or large-scale MIMO, as it is also called, is a MIMO system, with a very large number of antennas at the BS, in order to multiplex messages to several users in the same time-frequency resource [17]. The antennas focus on radiating power towards desired directions, bringing benefits in terms of EE and minimizing interference between cells [18].

As any other technology, massive MIMO has a good number of advantages and challenges that need to be overcome as a means to provide a good experience for all users in the network. In terms of benefits, it can dramatically improve capacity and have inexpensive infrastructure. In addition, it can exploit reduced latency and increased robustness to deal with interference [19]. 
Regarding its challenges, many have been analysed over the years, such as pilot contamination, hardware impairments, modulation and channel characterization $[19,20]$. Today, massive MIMO has been proven to enhance the reliability and throughput of wireless networks.

In recent years, many practical experiments have been carried out in order to test the potential of massive MIMO. In [21], it has been described some of the trials done until that point, where the technology has been explored below $6 \mathrm{GHz}$. The experiments could verify the gains theoretically expected, as well as demonstrate reciprocity based operation in mobile scenarios with users moving in higher speed (above $50 \mathrm{~km} / \mathrm{h}$ ). Big companies such as Huawei, Ericsson, Nokia, Samsung and even Facebook have reported their own tests with large-scale arrays. Not only massive MIMO has been implemented in cellular systems but also in Wi-Fi technology. Aligned to 5G, Wi-Fi has started using massive MIMO in indoor applications and unlicensed spectrum [22].

Within the possible setups for massive MIMO we have CAS and DAS. In a multicell environment with CAS, a BS is co-located at the center of each cell, with the advantage of low backhaul requirement. In contrast, in DAS systems, RRHs are spread all over the cell and connected to a CPU at a BS by optical fibers, coaxial cables or microwave [4].

Potential and obstacles of DAS have been thoroughly explored in multicellular systems. In [23], it has been proven that DAS can perform well with reduced power and increase system capacity while reducing interference from other cells and providing greater improvements for users near cell boundaries. A multiuser DAS setup has been examined as well as resource allocation techniques based on channel coefficients between RRHs and users [24]. Besides, it was pointed out that by using an appropriate power allocation, users just need to be connected to a few RRHs.

For the $5 \mathrm{G}$ of wireless systems, DAS are being considered with a focus on providing higher coverage probability, flexible resource management, higher power efficiency and larger capacities by the exploitation of smaller distances between BSs and users, and of spatial diversity [25].

As another direction on MIMO technology, virtual MIMO, coordinated multi-point joint processing (CoMP) and network MIMO have been proposed, [26-29], aiming to improve SE and diminish inter-cell interference. The idea is that BSs act together as a single antenna array and users can receive their desired signals through BSs close to them. By choosing correctly the antenna outputs, interference can be minimized and the system capacity increased [6].

Backhaul traffic, synchronization and feedback have been numbered out as some of the challenges brought by these technologies. However, due to its 
ability to mitigate interference between cells, BSs and users, network MIMO can achieve significant gains in both the uplink and downlink [30]. Furthermore, it has been reported that virtual MIMO can obtain additional multiuser diversity gains by user clustering, [31].

By capitalizing on the benefits of cooperative transmissions, at the cost of acquiring CSI and sharing the transmit data, virtual MIMO can greatly improve systems performance [7]. This cooperation helps mitigate inter-cell interference by jointly processing the user data in multiple BSs. This type of network does not necessarily need more antennas and offer advantages over simpler beamforming coordination schemes. Its extra complexity is due to the additional information being exchanged between users, BSs and CPUs participating in the cooperation [26].

The combination of massive MIMO with DAS systems and of virtual MIMO with DAS has been tested in the past, and proved that distributed setups can achieve higher rates than CAS [7,32]. In [33], it is indicated that large-scale DAS (L-DAS) systems are energy-efficient and have simpler precoding and power control, when associated with antenna selection and user clustering methods.

As an evolution of massive MIMO, DAS and virtual MIMO, in CellFree Massive MIMO, many randomly distributed APs are connected to a CPU and serve simultaneously a much smaller number of users. At the CPU, precoding techniques and power allocation algorithms can be performed. The main goal is to use advanced backhaul to provide a good experience for all users [34]. Compared to a cellular system, cell-free concepts have been shown to increase EE and per-user throughput, both in rural and urban scenarios [35]. Moreover, they can have simple signal processing which facilitates the usage of phenomena such as favorable propagation with channel hardening to their own advantage [3].

Many precoding and receive processing schemes have been previously designed for cell-free networks. In the uplink, a matched filter (MF) has been examined in [8], whereas in [10,36], an MMSE and large-scale fading decoding (LSFD) receivers have been demonstrated to provide higher outage rate than the former. On the other hand, in the downlink, a CB precoder has been investigated for computationally simple signal processing, at the cost of lower performance $[8,9,37]$. With more backhaul requirements, a ZF precoding design has been extensively used with the objective of improving system performance [9] and of maximizing the EE [38].

Uniformly good service for all users is a key point of cell-free systems, leading to the combination of many beamforming and detection techniques 
with power control algorithms. Different criteria have been used in the literature to satisfy certain aspects of the network. In [39], power allocation has been performed on the uplink to maximize the minimum user rate under per-user power constraints. An MF receiver and a $\mathrm{CB}$ precoder have been combined with an optimization that maximizes the smallest of all user rates under perAP constraints, [8]. Similarly, CB and ZF precoders were combined with the maximization of the minimum SINR from all users under per-AP power constraints in [9]. With a different criterion, ZF precoders were combined with EE maximization, under a per-AP power constraint and a per-user SE constraint, [37], taking into account backhaul power consumption and imperfect CSI [38].

To decrease the energy consumption in cell-free systems, some works have indicated APS, where each user is served by a subset of APs. In [2], two APS schemes have been proposed, one based on the received power and the other on the largest LS coefficients. Most works addressing Cell-Free Massive MIMO systems consider single-antenna APs. Nevertheless, multiple-antenna APs have been analysed with the intention of improving channel hardening and increasing the likelihood of favorable propagation occurence [40]. Additionally, it has been concluded that, in terms of costs, it is better to add more antennas to an AP than to install more APs [41] .

Since the seminal work that introduced the cell-free concept [8], imperfect CSI has been taken into account in most papers of the literature, as to provide a more realistic analysis of the scenario. As previously applied to MIMO systems and sensor arrays, robust techniques have been developed with an eye toward interference and CSI estimation error mitigation. Although never applied to cell-free before, robustness can be a useful tool to improve the performance of current systems, without much alteration in computational complexity.

In sensor array signal processing, robust adaptive beamforming has been carefully investigated to deal with random steering vector errors. One of the most famous techniques to treat mismatch errors and random perturbations is called diagonal load (DL). During a long time, there were no established rules on how to correctly acquire optimal values to apply this technique. Many works have explored this issue, being suggested in [42] that the parameters for DL can be precisely calculated based on the uncertainty of steering vectors, when applied to a Capon beamformer. Similarly, a variable loading strategy has been proposed to the same beamforming technique as a means to provide on demand loading instead of fixed or continuous [43]. We can as well mention a study where DL coefficients were found through spatial-matched method aiming to deal with covariance matrix mismatch and steering vector error in 
large-scale arrays [44].

Despite the obvious benefits of DL, the literature also addresses to other loading methods. In [45], a class of beamformers has been derived based on generalized loading, which does not necessarily present real advantages when compared to diagonal, but can be effective in some special cases.

When applied to MIMO systems, robust precoders have enabled the analysis of several classes of channel uncertainty. A robust MIMO precoder has been calculated based on the maximum worst-case received SINR or on the minimum worst-case error probability in [46]. In multicell scenarios, coordinated beamforming has been used with the purpose of mitigating interference between cells and providing quality of service (QoS). However, to treat channel uncertainties in these systems, and supposing constrained CSI, robust precoders applying the worst-case methodology have been provided in order to maximize the worst-case weighted sum-rate and the minimum worstcase rate and to minimize the weighted sum power of base stations subject to the worst-case SINR of users. Centralized and distributed processing have been considered $[47,48]$.

Aiming to treat the high complexity of these well-known approaches (DL and worst-case), dimensionality schemes have been investigated for the purpose of decreasing the complexity of the algorithms and improving convergence rate. In [49], a low-rank technique is presented where the cross-correlation between the array data and the output of the beamformer is exploited. To update the steering vector mismatch, this cross-correlation is projected into a Krylov subspace. In addition, algorithms based on different gradients have been derived to update the beamforming weights, reducing computational cost. In the same way, cross-correlation methods have been applied to relay systems, where the correlation is explored between the system's output and received data from the relays at the destination [50]. The proposed distributed beamforming with low-rank and cross-correlation has the objective to maximize the output SINR under total relay power constraint.

Referring to robust precoders and specifically to the ones with MMSE strategy, [11-15], robustness has been further incorporated to them and has provided significant advances. In [13], a robust joint linear MMSE precoder and decoder which takes into account the statistics of the outdated CSI at the transmitter is proposed. An optimization has been performed in [15] by minimizing the maximum MSE, taking into consideration the channel estimation matrix and the channel estimation error matrix. Reformulating the problem into a min-min convex minimization problem enables the solution to be found in closed form. Similarly, in $[14,51]$, the same objective function is 
used, with a tolerance for the channel estimation error, yet with total power constraint.

Robust ZF and MMSE precoders have been applied to relay systems in the past considering channel quantization errors and total power constraint [12]. They conclude that to calculate the precoding vectors, we only need the channel direction information (CDI) feedback. Likewise, in [11], a robust MMSE precoder based on switched relaying is indicated, outperforming previous precoding techniques applied to relay systems. By creating all possibilities of matrix pairs, the BS and the relay station (RS) precoding matrices, a selection scheme based on the MSE criteria has been derived, in favor of the most suitable options. Not only ZF and MMSE precoders have exploited the advantages of robustness, but also the Tomlinson-Harashima precoder (THP). A multibranch strategy has been developed, combining a THP with a linear precoder in a relay system, which are jointly optimized to minimize the MSE, taking into account an MMSE detector at the destination [52].

\section{2}

\section{System Model}

The downlink of a Cell-Free Massive MIMO system is assumed with $L$ randomly distributed single-antenna $\operatorname{APs}(N=1)$ and $K$ single-antenna users, where $L N=M$ and $M>>K$. The total number of antenna elements is represented by $M$ and the number of antenna elements per AP is $N$. In this system, all APs are connected to a CPU and serve simultaneously all users, as shown in Fig. 2.1. Each AP obtains CSI and sends them to the CPU, which performs APS, precoding and power allocation whose parameters are then fed back to the APs.

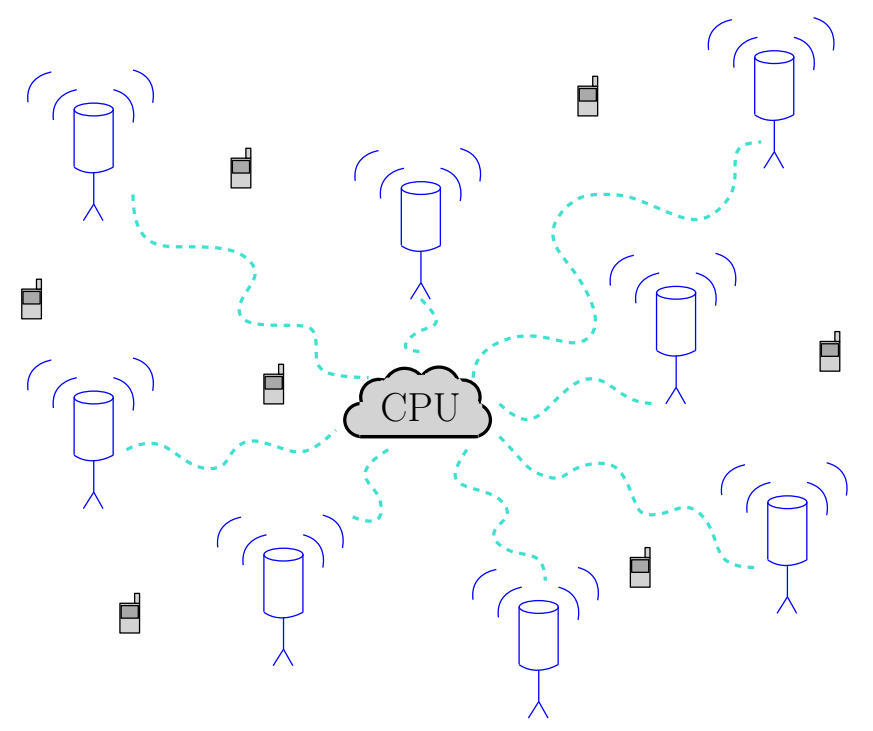

Figure 2.1: Cell-Free Massive MIMO System. 
The channel coefficients between the $m$ th antenna element and the $k$ th user are defined as [8]

$$
g_{m, k}=\sqrt{\beta_{m, k}} h_{m, k},
$$

where $\beta_{m, k}$ is the LS coefficient (path loss and shadowing effects) and $h_{m, k} \sim$ $\mathcal{C N}(0,1)$ is the small-scale fading coefficient, defined as independent and identically distributed (i.i.d) random variables (RVs) that remain constant during a coherence interval and are independent over different coherence intervals. Nonetheless, the LS coefficients change less frequently, being constant for several coherence intervals. Depending on the user's mobility, the LS coefficients may change at least 40 times slower than $h_{m, k}[8]$.

\subsection{1}

\section{Multiple-Antenna APs}

If, instead of assuming single-antenna APs as most works do, we decide to consider multiple-antenna APs [40,41], the system will have $L$ randomly distributed APs equipped with $N$ antenna elements each, where $L N=M$.

Regarding that each AP has $N$ antenna elements, we know that for the $l$ th AP

$$
\beta_{(l-1) \cdot N+1, k}=\beta_{(l-1) \cdot N+2, k}=\cdots=\beta_{l \cdot N, k}, \text { for } l=1, \ldots, L,
$$

where we suppose that the links between the antenna elements of an AP and the users have the same distance and are affected by the same path loss and shadowing effects as illustrated by

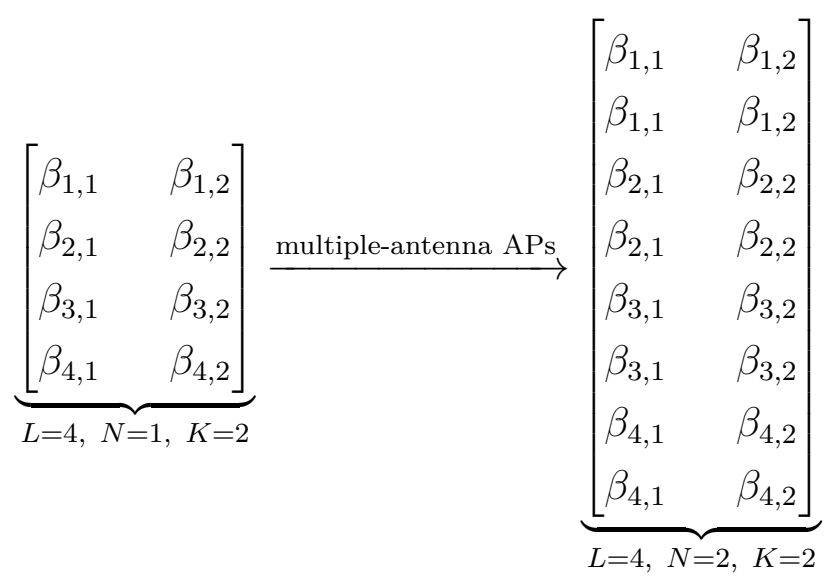

\subsection{2}

\section{Large Scale Fading Coefficient Model}

Considering $M$ antenna elements in total and $K$ single-antenna users uniformly distributed at random, the LS coefficients from (2-1) are modelled by

$$
\beta_{m, k}=\mathrm{PL}_{m, k} \cdot 10^{\frac{\sigma_{s h} z_{m, k}}{10}}
$$


where $\mathrm{PL}_{m, k}$ is the path loss and $10^{\frac{\sigma_{s h} z_{m, k}}{10}}$ refers to shadow fading, [8], with

$$
\begin{aligned}
& \sigma_{s h}=8 \mathrm{~dB} \\
& z_{m, k} \sim \mathcal{N}(0,1) .
\end{aligned}
$$

The path loss is based on a three-slope model [53], in $\mathrm{dB}$, described as

$$
\mathrm{PL}_{m, k}=\left\{\begin{array}{l}
-L-35 \log _{10}\left(d_{m, k}\right), \text { if } d_{m, k}>d_{1} \\
-L-15 \log _{10}\left(d_{1}\right)-20 \log _{10}\left(d_{m, k}\right), \text { if } d_{0}<d_{m, k} \leq d_{1} \\
-L-15 \log _{10}\left(d_{1}\right)-20 \log _{10}\left(d_{0}\right), \text { if } d_{m, k} \leq d_{0}
\end{array}\right.
$$

where

$$
\begin{aligned}
L \triangleq & 46.3+33.9 \log _{10}\left(f_{\text {freq }}\right)-13.82 \log _{10}\left(h_{\mathrm{AP}}\right) \\
& \quad-\left(1.1 \log _{10}\left(f_{\text {freq }}\right)-0.7\right) h_{\mathrm{u}}+\left(1.56 \log _{10}\left(f_{\text {freq }}\right)-0.8\right), \\
d_{0}= & 10 \mathrm{~m}, \\
d_{1}= & 50 \mathrm{~m}, \\
f_{\text {freq }}= & 1900 \mathrm{MHz}, \\
h_{\mathrm{AP}}= & 15 \mathrm{~m}, \\
h_{\mathrm{u}}= & 1.65 \mathrm{~m},
\end{aligned}
$$

$f_{\text {freq }}$ is the carrier frequency in $\mathrm{MHz}, h_{\mathrm{AP}}$ is the $\mathrm{AP}$ antenna height in meters, $h_{\mathrm{u}}$ is the user antenna height in meters, and $d_{m, k}$ is the distance between the $m$ th antenna element and the $k$ th user, as in [8]. When $d_{m, k} \leq d_{1}$ there is no shadowing.

\subsection{3}

\section{Channel Estimation}

For the purpose of reducing interference between signals intended for different users, bearing in mind that each user is served by all APs, channel coefficients need to be taken into account when forming transmitted signals. The system employs the TDD protocol, where the channel is estimated through uplink training. Assuming TDD operation together with the perfect calibration of hardware chains, channel reciprocity can be considered, meaning that the channel coefficients for the uplink and downlink are the same [8]. To begin with, all users send simultaneously and synchronously pilot sequences, $\boldsymbol{\Pi}_{1}, \ldots, \boldsymbol{\Pi}_{K} \in \mathbb{C}^{\tau}$, to each antenna, where $\left\|\boldsymbol{\Pi}_{K}\right\|^{2}=1$ and $\tau$ is the length of pilot sequences. Then, APs perform MMSE channel estimation and obtain $\hat{g}_{m, k}$, the estimate of the channel coefficient $g_{m, k}$ between the $m$ th antenna and the $k$ th user. With $\hat{g}_{m, k}$, the data are transmitted to all users. 
Supposing a carrier frequency of $1.9 \mathrm{GHz}$ and low user's mobility, the coherence interval is large and many orthogonal pilots can be used in channel estimation. As a result, we assume that the pilot sequences assigned to the corresponding users are orthonormal. Taking into account that the same pilot is assigned to users far away from each other, pilot contamination is assumed negligible [9]. On the contrary, if we increased the users' mobility, we would have to consider interference caused by pilot contamination given that the number of orthogonal pilots available would be much smaller. In Fig. 2.2, a description of the TDD protocol is illustrated.

\begin{tabular}{|c|c|c|}
\hline$\tau_{r p}$ & 1 & $T-1-\tau_{r p}$ \\
\hline reverse pilots $\rceil_{\text {computation }}$ forward QPSK symbols
\end{tabular}

Figure 2.2: TDD Protocol

In the training step, the $m$ th antenna receives

$$
\mathbf{y}_{m}=\sqrt{\rho_{r} \tau} \sum_{k=1}^{K} g_{m, k} \boldsymbol{\Pi}_{k}+\mathbf{w}_{m}
$$

where $\rho_{r}$ is the uplink power and $\mathbf{w}_{m} \sim \mathcal{C N}\left(0, \mathbf{I}_{\tau}\right)$ is the additive noise.

The MMSE estimate of $g_{m, k}$ can be expressed as

$$
\hat{g}_{m, k}=\frac{\sqrt{\rho_{r} \tau} \beta_{m, k}}{1+\rho_{r} \tau \beta_{m, k}} \boldsymbol{\Pi}_{k}^{H} \mathbf{y}_{m}
$$

Due to channel hardening in massive MIMO systems [17], we infer that each user is only aware of the statistics of the estimated channel coefficients. Then, the variance of the estimate, $\mathbb{E}\left(\left|\hat{g}_{m, k}\right|^{2}\right)$, is equal to

$$
\alpha_{m, k}=\frac{\rho_{r} \tau \beta_{m, k}^{2}}{1+\rho_{r} \tau \beta_{m, k}} .
$$

Since

$$
\tilde{g}_{m, k}=g_{m, k}-\hat{g}_{m, k}
$$

where $\tilde{g}_{m, k}$ is the CSI error between the $m$ th antenna element and the $k$ th user, we write

$$
\hat{g}_{m, k} \sim \mathcal{C N}\left(0, \alpha_{m, k}\right), \quad \tilde{g}_{m, k} \sim \mathcal{C N}\left(0, \beta_{m, k}-\alpha_{m, k}\right) .
$$

We notice that $\alpha_{m, k}$ as well as $\hat{g}_{m, k}$ are functions of $\beta_{m, k}$. Consequently, to evaluate perfect and different levels of imperfect CSI we set $\alpha_{m, k}$ as an adjustable percentage of $\beta_{m, k}(0 \leq n \leq 1)$. Thus, we get 


$$
\begin{aligned}
\alpha_{m, k} & =n \beta_{m, k} \\
\tilde{g}_{m, k} & =g_{m, k}-\hat{g}_{m, k}, \text { and } \\
\mathbb{E}\left[\left|\tilde{g}_{m, k}\right|^{2}\right] & =(1-n) \beta_{m, k} .
\end{aligned}
$$

\subsection{4}

\section{Downlink Payload Data Transmission}

After channel estimation is carried out, the data are transmitted to all users. The signal received by the $k$ th user is described by

$$
y_{k}=\sqrt{\rho_{f}} \mathbf{g}_{k}^{T} \mathbf{P} \mathbf{s}+w_{k}
$$

where $\rho_{f}$ is the maximum transmitted power of each antenna, $\mathbf{g}_{k}=$ $\left[g_{1, k}, \ldots, g_{M, k}\right]^{T}$ are the channel coefficients for user $k, \mathbf{P} \in \mathbb{C}^{M \times K}$ is a generic precoding matrix, $\mathbf{s}=\left[s_{1}, \ldots, s_{K}\right]^{T}$ is the zero mean symbol vector, with $\sigma_{s}^{2}=\mathbb{E}\left(\left|s_{k}\right|^{2}\right), s_{k}$ is the data symbol intended for user $k, w_{k} \sim \mathcal{C N}\left(0, \sigma_{w}^{2}\right)$ is the additive noise for user $k$ and $\sigma_{w}^{2}$ is the noise variance. We presume that the elements of $\mathbf{s}$ are mutually independent, and independent of all noise and channel coefficients.

For all users combined, we have the following model

$$
\mathbf{y}=\sqrt{\rho_{f}} \mathbf{G}^{T} \mathbf{P s}+\mathbf{w},
$$

where $\mathbf{G} \in \mathbb{C}^{M \times K}$ is the channel matrix with elements $[\mathbf{G}]_{m, k}=g_{m, k}$ and $\mathbf{w}=\left[w_{1}, \ldots, w_{K}\right]^{T}$ is the noise vector.

Due to the strong path loss characteristic of Cell-Free Massive MIMO systems, $\rho_{f}$ takes the form of [54]

$$
\rho_{f}=\frac{\mathrm{SNR} \cdot \operatorname{tr}\left(\mathbf{C}_{w}\right)}{\mathbb{E}\left[\|\hat{\mathbf{G}}\|_{F}^{2}\right]}=\frac{\mathrm{SNR} \cdot K \sigma_{w}^{2}}{\operatorname{tr}\left(\hat{\mathbf{G}} \hat{\mathbf{G}}^{H}\right)},
$$

where

$$
\begin{aligned}
\sigma_{w}^{2} & =T_{0} \times k_{B} \times B \times \mathrm{NF}(\mathrm{W}), \\
T_{0} & =290 \mathrm{~K}, \\
k_{B} & =1.381 \times 10^{-23} \mathrm{~J} / \mathrm{K}, \\
B & =20 \mathrm{MHz}, \\
\mathrm{NF} & =9 \mathrm{~dB},
\end{aligned}
$$

$[\hat{\mathbf{G}}]_{m, k}=\hat{g}_{m, k}$ is the CSI matrix, $\mathbf{C}_{w}$ is the noise covariance matrix, $T_{0}$ is the noise temperature in Kelvin, $k_{B}$ is the Boltzmann constant in Joule per Kelvin, $B$ is the bandwidth in $\mathrm{Hz}$ and $\mathrm{NF}$ is the noise figure in Watts.

Hence, the SNR is written as 


$$
\mathrm{SNR}=\frac{\rho_{f} \mathbb{E}\left[\|\hat{\mathbf{G}}\|_{F}^{2}\right]}{\operatorname{tr}\left(\mathbf{C}_{w}\right)}=\frac{\rho_{f} \operatorname{tr}\left(\hat{\mathbf{G}} \hat{\mathbf{G}}^{H}\right)}{K \sigma_{w}^{2}} .
$$

In this work, we will compare different precoding designs combined with APS and power allocation techniques, so an abstract SNR is mandatory. Note that the expression above does not take in account the beamforming gain. Thus, the resulting SINR of all the techniques studied can be higher than the SNR in the numerical results [55].

\section{3}

\section{Precoding and Power Allocation Techniques}

Here we describe the two precoding techniques that were already created for Cell-Free Massive MIMO Systems: CB and ZF, both taken from [8,9].

\subsection{1}

\section{Conjugate Beamforming}

First, the $l$ th AP estimates $\beta_{m, k}, k=1, \ldots, K$ and sends them to the CPU. Then, the CPU computes the power coefficients $\eta_{m, k}, m=1, \ldots, M$, $k=1, \ldots, K$ as a function of $\beta_{m, k}$ and sends these coefficients to corresponding APs. In parallel, users are sending pilot sequences to the $l$ th AP so that they can get the channel estimates $\hat{g}_{m, k}, k=1, \ldots, K$.

With this approach, the $m$ th antenna transmits the following signal

$$
x_{m}=\sqrt{\rho_{f}} \sum_{i=1}^{K} \sqrt{\eta_{m, i}} \hat{g}_{m, i}^{*} s_{i},
$$

where $\eta_{m, i}$ is the power coefficient used by the $m$ th antenna for transmission to user $i, \hat{g}_{m, i}^{*}$ is the conjugate of the estimated channel coefficient between the $m$ th antenna and user $i$ and $s_{i}$ is the symbol intended for user $i$.

The signal received by the $k$ th user is

$$
y_{k}=\sum_{m=1}^{M} g_{m, k} x_{m}+w_{k} \text {. }
$$

The achievable rate of user $k$ assuming Gaussian signalling in the downlink has been described in [9] as

$$
R_{\mathrm{k}, \mathrm{CB}}=\log _{2}\left(1+\mathrm{SINR}_{\mathrm{k}, \mathrm{CB}}\right)
$$

where

$$
\mathrm{SINR}_{\mathrm{k}, \mathrm{CB}}=\frac{\rho_{f}\left(\sum_{m=1}^{M} \sqrt{\eta_{m, k}} \alpha_{m, k}\right)^{2}}{\sigma_{w}^{2}+\rho_{f} \sum_{i=1}^{K} \sum_{m=1}^{M} \eta_{m, i} \beta_{m, k} \alpha_{m, i}}
$$




\subsubsection{1}

\section{Optimal Power Allocation (OPA)}

In $[8,9]$, an optimal power allocation is suggested for the CB precoder. We would like to find the $\eta_{m, k}, m=1, \ldots, M, k=1, \ldots, K$ that maximizes the minimum $\operatorname{SINR}_{\mathrm{k}, \mathrm{CB}}, k=1, \ldots, K$ under the constraint that the transmitted power of each antenna is limited by $\rho_{f}$.

The expected transmitted power of the $m$ th antenna is equal to

$$
\mathbb{E}\left(\left|x_{m}\right|^{2}\right)=\rho_{f} \sum_{i=1}^{K} \eta_{m, i} \alpha_{m, i}
$$

Then, the max-min problem is formulated as follows:

$$
\begin{aligned}
& \max _{\eta} \min _{k} \operatorname{SINR}_{\mathrm{k}, \mathrm{CB}}(\boldsymbol{\eta})=\frac{\rho_{f}\left(\sum_{m=1}^{M} \sqrt{\eta_{m, k}} \alpha_{m, k}\right)^{2}}{\sigma_{w}^{2}+\rho_{f} \sum_{i=1}^{K} \sum_{m=1}^{M} \eta_{m, i} \beta_{m, k} \alpha_{m, i}} \\
& \text { s.t. } \sum_{i=1}^{K} \eta_{m, i} \alpha_{m, i} \leq 1, m=1, \ldots, M .
\end{aligned}
$$

Since the numerator of $\mathrm{SINR}_{\mathrm{k}, \mathrm{CB}}$ is a concave function and the denominator is a linear function of $\boldsymbol{\eta}$, the problem (2-24) is quasiconcave, which requires the application of the bisection method approached in [1]. Despite this, before, the problem is put in an epigraph form:

$$
\begin{aligned}
& \max _{\boldsymbol{\eta}, t} t \\
& \text { s.t. } \operatorname{SINR}_{\mathrm{k}, \mathrm{CB}}(\boldsymbol{\eta}) \geq t, k=1, \ldots, K, \text { and } \\
& \qquad \sum_{i=1}^{K} \eta_{m, i} \alpha_{m, i} \leq 1, m=1, \ldots, M .
\end{aligned}
$$

By setting a fixed $t$ inside an interval $\left(t_{b}, t_{e}\right)$, the domain of constraints in (2-25) is convex and then it needs to be decided if for a certain $t$ the problem is feasible or not. The bisection method is explained in Algorithm 1.

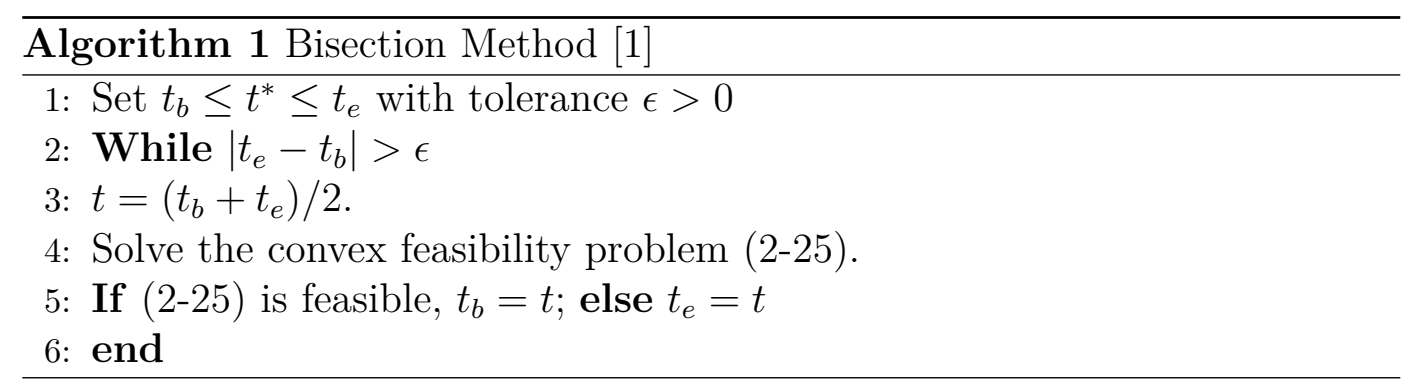

The complexity of this algorithm is high. For this reason we will compare its performance with the case of a Uniform Power Allocation (UPA), derived in a few moments. 
To perform this optimization problem in CVX, a different formulation is needed. Therefore, we suggest that (2-25) is reformulated as a second-order cone program (SOCP) [1].

After doing several manipulations in (2-24a), we obtain the following:

$$
\sqrt{\frac{\rho_{f}}{t}}\left(\eta_{m, k}^{\prime \prime}\right)^{T} \alpha_{m, k} \geq\left(\sqrt{\sigma_{w}^{2}+\rho_{f} \sum_{i=1}^{K}\left(\left(\eta_{m, i}^{\prime \prime}\right)^{T} \operatorname{diag}\left(\beta_{m, k} \alpha_{m, i}\right) \eta_{m, i}^{\prime \prime}\right)}\right)
$$

where $\eta_{m, k}^{\prime \prime}=\sqrt{\eta_{m, k}}$.

To transform the expression above into a SOCP form, we do, for the case of $K=2$, as the example:

$$
\begin{aligned}
& \text { minimize } f^{T} x \\
& \text { subject to }\left\|A_{i} x+b_{i}\right\|_{2} \leq c_{i}^{T} x+d_{i}, i=1, \ldots, m \\
& \quad F x=g
\end{aligned}
$$

where

$$
A_{i}=\left[\begin{array}{ccccccc}
\sqrt{\rho_{f} \beta_{1, K} \alpha_{1,1}} & & \ldots & 0 & 0 & 0 & 0 \\
\vdots & \ddots & 0 & 0 & 0 & 0 & 0 \\
0 & 0 & \sqrt{\rho_{f} \beta_{M, K} \alpha_{M, 1}} & 0 & 0 & 0 & 0 \\
0 & 0 & 0 & \sqrt{\rho_{f} \beta_{1, K} \alpha_{1,2}} & 0 & 0 & 0 \\
0 & 0 & 0 & 0 & \ddots & 0 & 0 \\
0 & 0 & 0 & 0 & 0 & \sqrt{\rho_{f} \beta_{M, K} \alpha_{M, 2}} & 0 \\
0 & 0 & 0 & 0 & 0 & 0 & 0
\end{array}\right],
$$

$x=\left[\begin{array}{llllll}\eta_{1,1}^{\prime \prime} & \ldots & \eta_{M, 1}^{\prime \prime} & \eta_{1,2}^{\prime \prime} \ldots & \eta_{M, 2}^{\prime \prime} & 0\end{array}\right]_{(1 \times(M * K)+1)}^{T}$,

$c_{i}=\left[\begin{array}{llllll}\sqrt{\frac{\rho_{f}}{t}} \alpha_{1,1} & \ldots & \sqrt{\frac{\rho_{f}}{t}} \alpha_{M, 1} & 0 & \ldots & 0\end{array}\right]_{(1 \times(M * K)+1)}^{T}$,

$b_{i}=\left[\begin{array}{llll}0 & \ldots & 0 & \sigma_{w}\end{array}\right]_{(1 \times(M * K)+1)}^{T}$,

$d_{i}=0$.

\subsubsection{2}

\section{Uniform Power Allocation (UPA)}

A UPA scheme is suggested in [9], in the interest of reducing the complexity of the algorithm without loosing much of the performance. Here we suppose that the $m$ th antenna transmits with fixed power $p_{m}\left(\beta_{m}^{\max }\right)=e^{\left(-\kappa \beta_{m}^{\max }\right)}$ and that $\eta_{m, k}=\eta_{m}$, for $k=1, \ldots, K$, which means that the power coefficients are the same for all users. The power coefficients are calculated directly from 
the per-antenna power constraint as

$$
\eta_{m}=\frac{p_{m}\left(\beta_{m}^{\max }\right)}{\sum_{i=1}^{K} \alpha_{m, i}},
$$

where $\beta_{m}^{\max }=\max _{k} \beta_{m, k}$. The coefficient $\kappa$ is chosen to best fit the exponential function, given a certain $M$ and $K$, as detailed in [9].

\subsection{2}

\section{Zero Forcing}

As performed in the $\mathrm{CB}$ design, in the $\mathrm{ZF}$ technique, the $l$ th $\mathrm{AP}$ estimates $\beta_{m, k}, k=1, \ldots, K$ and sends them to the CPU. The CPU computes the power coefficients $\eta_{m, k}, m=1, \ldots, M, k=1, \ldots, K$ as a function of $\beta_{m, k}$. In parallel, users send pilot sequences to the $l$ th AP so that they can get the channel estimates $\hat{g}_{m, k}, k=1, \ldots, K$. Differently from CB, the estimated channel coefficients are sent to the CPU and put together with the power coefficients, $\eta_{m, k}$ so as to form ZF precoding vectors that are sent back to the APs.

The most important fact about $\mathrm{ZF}$ is that the signal transmitted to a user does not create interference to other users [55]. In [9], a ZF precoder with power allocation was developed and is described by

$$
\mathbf{P}_{\mathrm{ZF}}=\hat{\mathbf{G}}^{*}\left(\hat{\mathbf{G}}^{T} \hat{\mathbf{G}}^{*}\right)^{-1} \mathbf{N}
$$

where $\mathbf{N}$ is the power allocation matrix. To avoid interference, instead of having entries $\eta_{m, k}, \mathbf{N}$ will be a diagonal matrix where its coefficients are only function of $k$, such as $\eta_{m, k}=\eta_{k}, k=1, \ldots, K$. Then, $\mathbf{N}$ is a diagonal matrix with $\sqrt{\eta_{1}}, \ldots, \sqrt{\eta_{K}}$ on its diagonal. The $k$ th user receives

$$
y_{k}=\sqrt{\rho_{f}} \mathbf{g}_{k}^{T} \mathbf{P}_{\mathrm{ZF}} \mathbf{s}+w_{k} .
$$

An achievable rate of the $k$ th user with the $\mathrm{ZF}$ precoder is introduced in $[9]$ as

$$
\mathrm{R}_{\mathrm{k}, \mathrm{ZF}}=\log _{2}\left(1+\mathrm{SINR}_{\mathrm{k}, \mathrm{ZF}}\right)
$$

where

$$
\begin{gathered}
\operatorname{SINR}_{\mathrm{k}, \mathrm{ZF}}=\frac{\rho_{f} \eta_{k}}{\sigma_{w}^{2}+\rho_{f} \sum_{i=1}^{K} \eta_{i} \gamma_{k, i}}, \\
\gamma_{k}=\operatorname{diag}\left\{\mathbb{E}\left(\left(\hat{\mathbf{G}}^{T} \hat{\mathbf{G}}^{*}\right)^{-1} \hat{\mathbf{G}}^{T} \mathbb{E}\left(\tilde{\mathbf{g}}_{k}^{*} \tilde{\mathbf{g}}_{k}^{T}\right) \hat{\mathbf{G}}^{*}\left(\hat{\mathbf{G}}^{T} \hat{\mathbf{G}}^{*}\right)^{-1}\right)\right\},
\end{gathered}
$$

$\tilde{\mathbf{g}}_{k}$ is the CSI error vector for user $k$ and $\mathbb{E}\left(\tilde{\mathbf{g}}_{k}^{*} \tilde{\mathbf{g}}_{k}^{T}\right)$ is a diagonal matrix with $(1-n) \beta_{m, k}$ on its $m$ th diagonal element. 


\subsubsection{1}

\section{Optimal Power Allocation (OPA)}

In the optimal power allocation for the ZF design, we would like to find $\eta_{k}, k=1, \ldots, K$ that maximizes the minimum $\operatorname{SINR}_{\mathrm{k}, \mathrm{ZF}}, k=1, \ldots, K$ under a certain constraint. Differently from CB, power coefficients are only functions of $k$ in order to cancel the interference to other users, meaning that $\eta_{1, k}=\cdots=\eta_{M, k}$.

The max-min problem can be formulated as follows:

$$
\begin{aligned}
& \max _{\eta} \min _{k} \operatorname{SINR}_{k, Z F}(\boldsymbol{\eta})=\frac{\rho_{f} \eta_{k}}{\sigma_{w}^{2}+\rho_{f} \sum_{i=1}^{K} \eta_{i} \gamma_{k, i}} \\
& \text { s.t. } \sum_{i=1}^{K} \eta_{i} \delta_{m, i} \leq 1, m=1, \ldots, M .
\end{aligned}
$$

where

$$
\boldsymbol{\delta}_{m}=\operatorname{diag}\left\{\mathbb{E}\left(\left(\hat{\mathbf{G}}^{T} \hat{\mathbf{G}}^{*}\right)^{-1} \hat{\mathbf{g}}_{m} \hat{\mathbf{g}}_{m}^{H}\left(\hat{\mathbf{G}}^{T} \hat{\mathbf{G}}^{*}\right)^{-1}\right)\right\},
$$

$\gamma_{k, i}$ is the $i$ th element of vector $\boldsymbol{\gamma}_{k}, \delta_{m, i}$ is the $i$ th element of vector $\boldsymbol{\delta}_{m}$ and $\hat{\mathbf{g}}_{m}$ is the CSI vector for the $m$ th antenna.

The numerator and denominator of $\operatorname{SINR}_{k, Z F}$ are linear functions of $\boldsymbol{\eta}$, meaning that (2-35) is quasilinear, which requires the employment of the bisection method introduced in [1]. The problem can be written in an epigraph form:

$$
\begin{aligned}
& \text { find } \boldsymbol{\eta} \\
& \text { s.t. } \operatorname{SINR}_{k, Z F}(\boldsymbol{\eta}) \geq t, k=1, \ldots, K, \\
& \qquad \sum_{i=1}^{K} \eta_{i} \delta_{m, i} \leq 1, m=1, \ldots, M .
\end{aligned}
$$

In this case, there was no need for reformulations and the problem could be automatically put in CVX.

\subsubsection{2}

\section{Uniform Power Allocation (UPA)}

In view of the fact that the OPA algorithm has a high complexity, we will compare its performance with the case of an UPA algorithm:

$$
\eta_{k}=1 /\left(\max _{m} \sum_{i=1}^{K} \delta_{m, i}\right), k=1, \ldots, K .
$$

Although it does not perform as well as the optimal approach, it is a nice alternative with lower computational complexity. 


\section{4}

\section{Transmit Wiener Filter for MIMO Systems}

Here we introduce the Wiener Filter (WF), [56], already designed for MIMO systems which will be used as a background for the techniques presented further in this work.

To begin with, we assume the following received signal vector for all users combined

$$
\mathbf{y}=\mathbf{E}\left(\mathbf{G}^{T} \mathbf{P s}+\mathbf{w}\right),
$$

where $\mathbf{E}$ is the receive filter.

To solve the problem, the following optimization is solved:

$$
\begin{aligned}
\left\{\mathbf{P}_{\mathrm{WF}}, f_{\mathrm{WF}}\right\} & =\operatorname{argmin}_{\{\mathbf{P}, f\}} \mathbb{E}\left[\left\|\mathbf{s}-f^{-1} \mathbf{y}\right\|_{2}^{2}\right] \\
\mathbb{E}\left[\|\mathbf{P s}\|_{2}^{2}\right] & =E_{t r} .
\end{aligned}
$$

For this problem, the solution is given by

$$
\mathbf{P}_{\mathrm{WF}}=f_{\mathrm{WF}} \mathbf{F}^{-1} \mathbf{G}^{*} \mathbf{E}^{H}
$$

where

and

$$
f_{\mathrm{WF}}=\sqrt{\frac{E_{t r}}{\operatorname{tr}\left(\mathbf{F}^{-2} \mathbf{G}^{*} \mathbf{E}^{H} \mathbf{C}_{s} \mathbf{E} \mathbf{G}^{T}\right)}}
$$

$$
\mathbf{F}=\mathbf{G}^{*} \mathbf{E}^{H} \mathbf{E G}+\frac{\operatorname{tr}\left(\mathbf{E C}_{w} \mathbf{E}^{H}\right)}{E_{t r}} \mathbf{I}_{M} .
$$

Later on, this optimization problem will be adapted to cell-free networks as well as integrated to robustness.

\section{5}

\section{Access Point Selection (APS)}

In this part we highlight the APS techniques already present in the literature, [2], which gave us the inspiration to develop our own methods. Both selection techniques are based on the $\mathrm{CB}$ precoder.

\subsection{1}

\section{Received-Power-Based Selection}

Firstly, we present an APS scheme based on the received power. The total received power of the desired signal of the $k$ th user becomes

$$
\mathrm{DS}_{k}=\sqrt{\rho_{f}} N \sum_{l=1}^{L} \sqrt{\eta_{l, k}} \alpha_{l, k}
$$

where the $l$ th AP contributes to $\sqrt{\rho_{f}} N \sqrt{\eta_{l, k}} \alpha_{l, k}$. Therefore, its contribution to the total received power of the $k$ th user is 


$$
\chi(l, k)=\frac{\sqrt{\eta_{l, k}} \alpha_{l, k}}{\sum_{i=1}^{L} \sqrt{\eta_{i, k}} \alpha_{i, k}} .
$$

A set of $\mathcal{A}_{k}$ is formed with the largest $\chi(l, k)$ until a certain tolerance

$$
\sum_{l \in \mathcal{A}_{k}} \chi(l, k) \geq \zeta \%
$$

By putting $\chi(l, k)$ in a descending order and choosing the largest until it reaches the tolerance, we find the set of selected APs. After choosing $\mathcal{A}_{k}, k=1, \ldots, K$ we can determine $\mathcal{U}_{l}$, which is the set of users served by the $l$ th AP. The procedure is better explained in Algorithm 2.

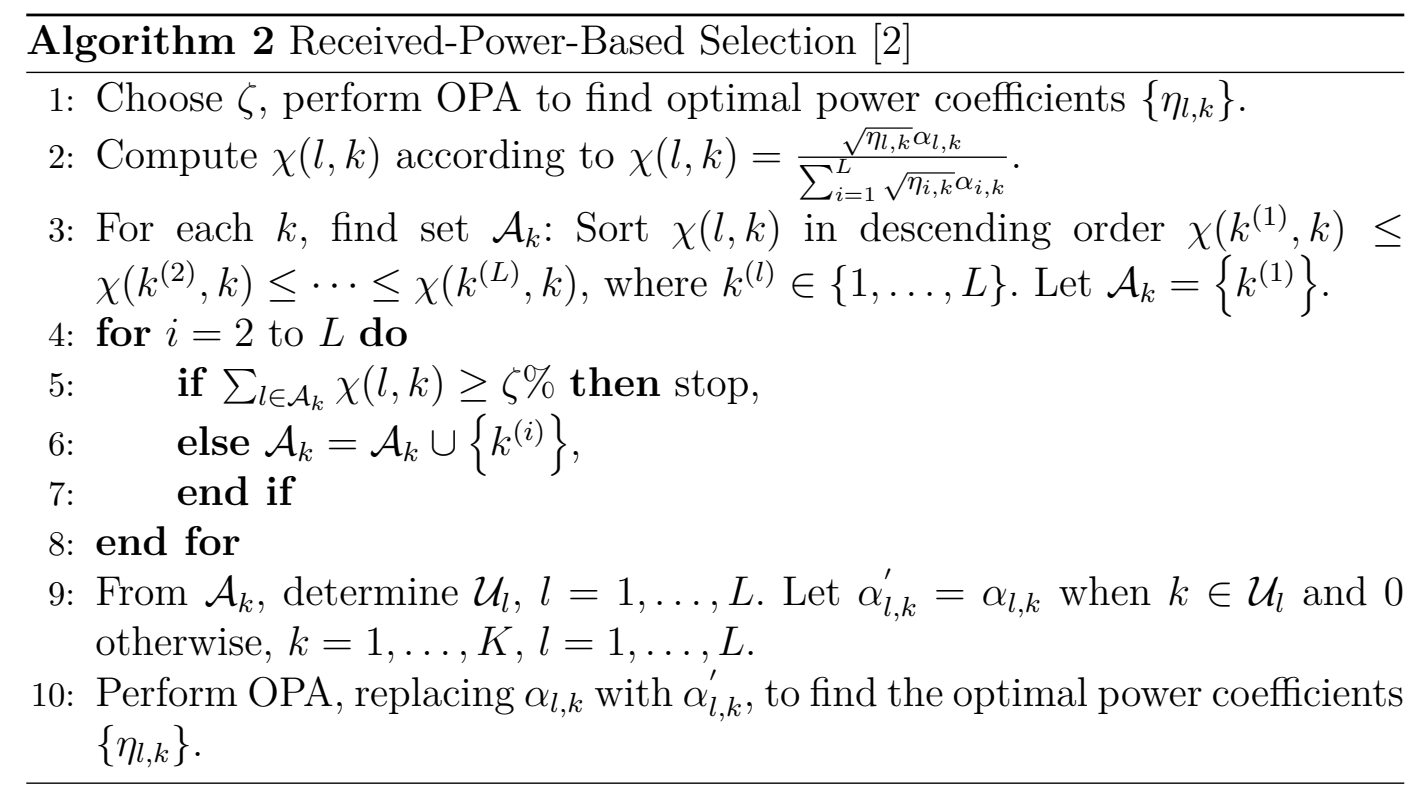

\section{5 .2}

\section{Largest-Large-Scale-Fading-Based Selection}

Furthermore, a largest LS coefficients technique is considered in [2]. The $k$ th user is associated to the $L_{0, k}<L$, where $L_{0, k}$ is the set of the largest LS coefficients. First, $\beta_{l, k}$ is sorted in a descending order, and the largest coefficients are chosen until their sum reaches a certain tolerance as expressed below:

$$
\sum_{l=1}^{L_{0, k}} \frac{\bar{\beta}_{l, k}}{\sum_{i=1}^{L} \beta_{i, k}} \geq \zeta \%,
$$

where $\bar{\beta}_{l, k}$ is the element of the sorted (in descending order) set of the set $\left\{\beta_{1, k}, \ldots, \beta_{L, k}\right\}$

In Algorithm 3, the method is described.

The benefit of the second scheme compared to the former is that it does not need to compute power coefficients $\eta_{l, k}$ before being performed. This reduces the complexity of the problem significantly. 


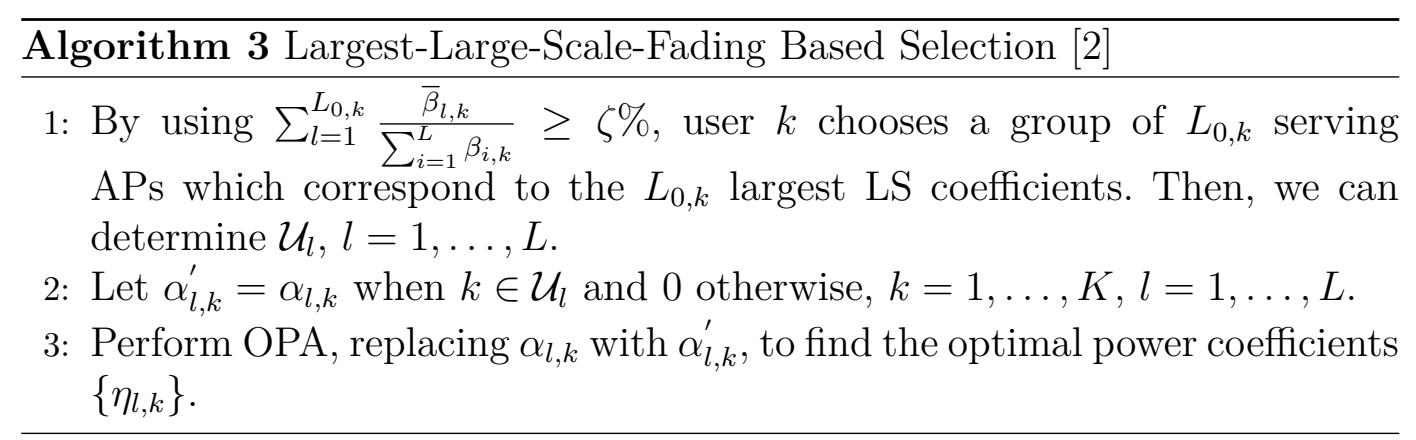

\section{6}

\section{Numerical Results}

Here, we showcase numerical results that compare the performance of the techniques and arrangements introduced in this chapter.

First, we illustrate in Fig. 2.3 the performance of a ZF precoder, in four different setups: Multi-cell CAS, Multi-cell DAS, Cell-Free and Multicell Network MIMO+CAS. In the multi-cell cases, we considered four cells. For a fair comparison, the total number of transmit antennas and users is the same in all scenarios.

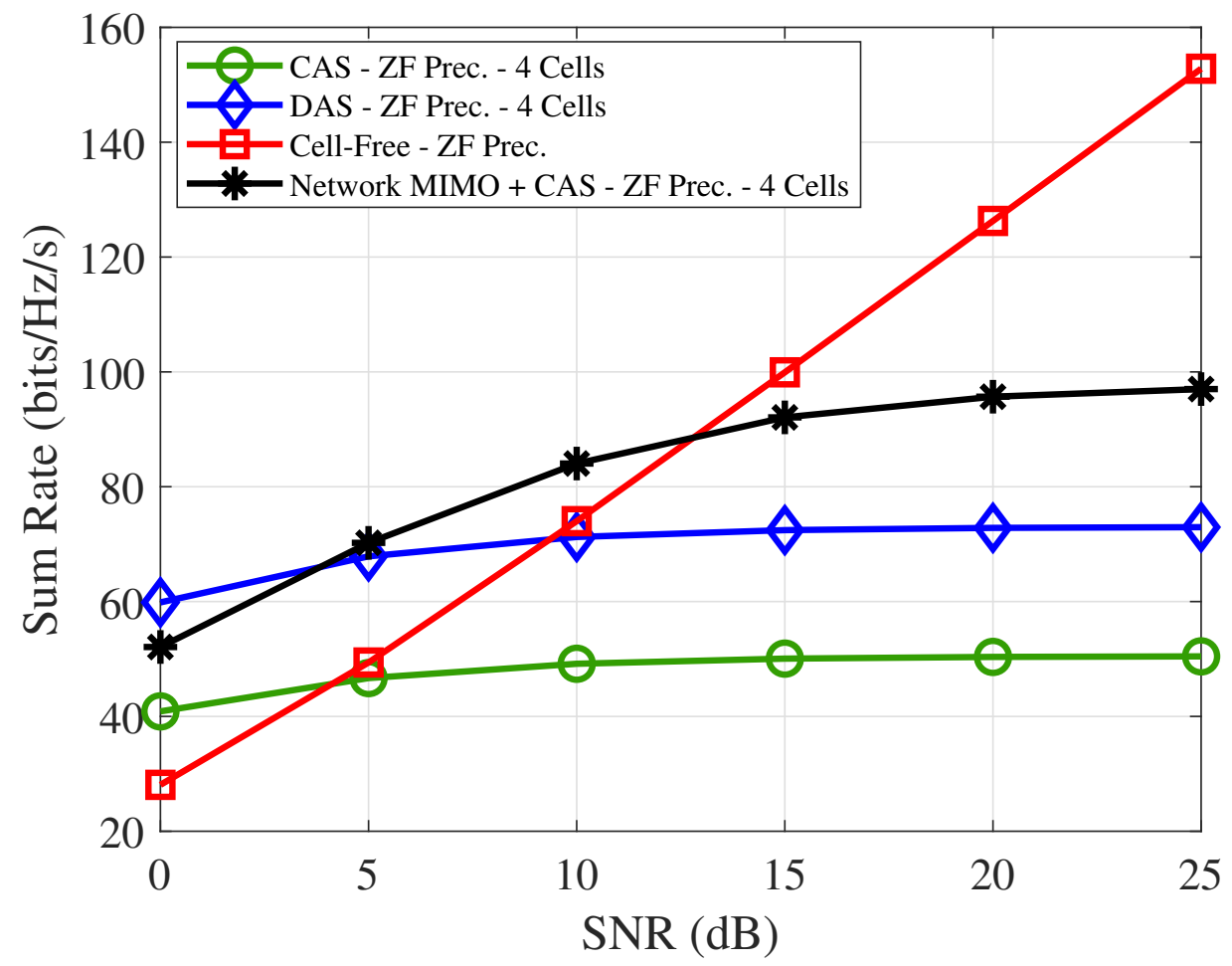

Figure 2.3: Sum-Rate vs. SNR with $L=128, \mathrm{~N}=1, K=16, n=1$, and 120 channel realizations.

We can observe that for higher SNR values, the cell-free system provides higher rates than other configurations. As expected, DAS outperforms CAS 
for all SNR values and having a cooperative network actually provides better results than to have a distributed arrangement.

The results collaborate with the theories exposed in [57], where it has been presented a comparison between centralized massive MIMO, network MIMO and Cell-free Massive MIMO systems. According to [57], cell-free provides improved uniform coverage and higher EE, when compared to others setups.

Next, we compare the CB precoder with the ZF precoder, both with UPA and OPA, as shown in Fig. 2.4. As we can see, the OPA algorithm in both cases perform much better than UPA and ZF provides better rates than those of CB.

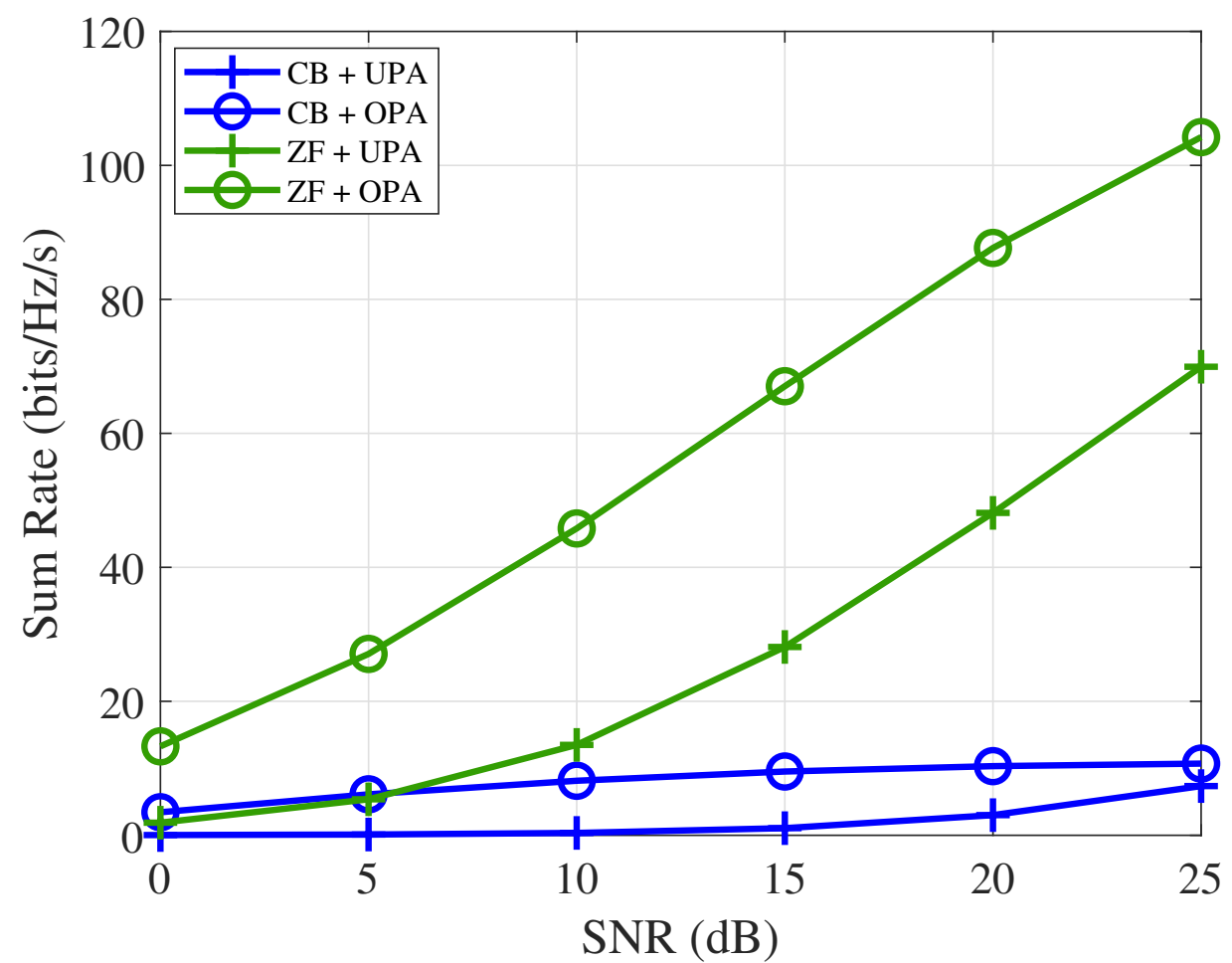

Figure 2.4: Sum-Rate vs. SNR with $L=128, N=1, K=16, n=0.99$ and 120 channel realizations

\section{7}

\section{Summary}

In this chapter we presented a review on the main topics that lead to the emergence of Cell-Free Massive MIMO Systems, as well as an introduction of important techniques developed over the years which can be perfectly applied to enhance the performance of this scenario, such as precoding, power allocation, APS and robust schemes. 
Then, we described the cell-free system model, together with an explanation on how to perform channel estimation and transmit data on the downlink. Additionally, we provide expressions to compute CB and ZF precoders combined with UPA and OPA techniques from $[8,9]$. The transmit WF, which is the foundation of this work, is presented and two APS strategies are explained. Numerical results are shown to better illustrate the performance of evaluated techniques. 


\section{3 \\ Iterative AP Selection, MMSE Precoding and Power Alloca- tion for Cell-Free Massive MIMO Systems}

In this chapter, we introduce two APS techniques, one based on ES and the other on LS coefficients. Next, an iterative linear MMSE precoding scheme is designed based on the total power constraint. Power allocation schemes based on max-min fairness and adaptive techniques are investigated to improve the performance of the proposed approach. Additionally, a sum-rate analysis is derived, based on the studied designs and the computational complexity of the methods is calculated for comparison purposes. Finally, numerical results of the developed schemes, are individually and integrally displayed.

In Section 3.1, we present APS, how to combine it with precoding, and the two techniques elaborated. Then, in Section 3.2, an MMSE precoder is described, based on the total transmit power. In Section 3.3, OPA, APA and UPA schemes are designed. Sum-rate analysis and the computational cost are evaluated in Section 3.4. Numerical results and discussions are presented in Section 3.5. Conclusions of this chapter are drawn in Section 3.6.

\section{1}

\section{Access Point Selection (APS)}

We describe two APS techniques for reducing power consumption, and consequently, increasing the EE in cell-free settings. To begin with, we explain how to perform APS in multiple precoding techniques, taking into consideration the power allocation problems that are examined in this work. An ES APS (ES-APS) scheme and a suboptimal LS coefficients based APS (LS-APS) with low computational cost are devised [2].

\subsection{1}

\section{Precoding with APS}

To perform APS with precoding, different approaches need to be used in the optimization problems. First, an auxiliary matrix $\mathbf{Q} \in \mathbb{N}^{M \times K}$, where $[\mathbf{Q}]_{m, k}=q_{m, k} \in\{0,1\}$ is defined in order to set which links between APs and users are taken into account (as 1) and the ones that are discarded (as 0). If, for an user $k$ the best set is to take APs 1,3 and 5, from a set of 5 APs, $q_{k}$ will 
be

$$
q_{k}=\left[\begin{array}{c}
1 \\
0 \\
1 \\
0 \\
1
\end{array}\right]
$$

where $q_{k}$ is the $k$ th column of $\mathbf{Q}$ associated to user $k$.

In this work, we examine the case of multiple-antenna APs as well. We perform APS rather than selecting antenna elements, meaning that all antennas from the $l$ th AP should be considered or discarded. As a consequence, the same procedure carried out for the LS coefficients $\left(\beta_{m, k}\right)$ is done for $q_{m, k}$ where, $q_{(l-1) \cdot N+1, k}=q_{(l-1) \cdot N+2, k}=\cdots=q_{l \cdot N, k}$, for $l=1, \ldots, L$. Now imagine a case where for an user $k$ the best set is to take APs 1,3 and 5, from a set of 5 APs with 2 antenna elements each. Then, $q_{k}$ will be

$$
q_{k}=\left[\begin{array}{c}
1 \\
1 \\
0 \\
0 \\
1 \\
1 \\
0 \\
0 \\
1 \\
1
\end{array}\right] .
$$

Before calculating the MMSE precoder, each coefficient $q_{m, k}$ will be multiplied by $\alpha_{m, k}, \beta_{m, k}, \hat{g}_{m, k}$ and $\tilde{g}_{m, k}$ so that $\alpha_{m, k}^{\prime}=q_{m, k} \cdot \alpha_{m, k}, \beta_{m, k}^{\prime}=q_{m, k}$. $\beta_{m, k}, \hat{g}_{m, k}^{\prime}=q_{m, k} \cdot \hat{g}_{m, k}$ and $\tilde{g}_{m, k}^{\prime}=q_{m, k} \cdot \tilde{g}_{m, k}$. Subsequently, precoding and power allocation algorithms are performed with these parameters. Similarly, we apply the same method to the ZF precoder from [9]. On the other hand, to perform APS in the CB precoder from [8,9], the max-min fairness power allocation algorithm turns into a mixed continuous/discrete optimization problem [58]. By annulling multiple coefficients in matrices $\boldsymbol{\alpha}, \boldsymbol{\beta}, \hat{\mathbf{G}}$ and $\tilde{\mathbf{G}}$, we considerably reduce the complexity of computing the $\mathrm{CB}, \mathrm{ZF}$ and MMSE precoders and their respective SINRs. To apply APS in future calculations, substitute $\boldsymbol{\alpha}, \boldsymbol{\beta}$, $\hat{\mathbf{G}}$ and $\tilde{\mathbf{G}}$ with $\boldsymbol{\alpha}^{\prime}, \boldsymbol{\beta}^{\prime}, \hat{\mathbf{G}}^{\prime}$ and $\tilde{\mathbf{G}}^{\prime}$. 


\subsection{2}

\section{Exhaustive Search Selection (ES-APS)}

In this scheme, all possible sets in a total of $L$ APs, $S$ selected APs and $K$ users, will be tested so that the combination that maximizes the minimum SINR is chosen. Each possible vector will be a column of matrix $\mathbf{V}$, formed by $S$ 1s and $(L-S)$ 0s. By choosing a set of $K$ columns of matrix $\mathbf{V}$, performing all combinations between them, $\left(\begin{array}{l}L \\ S\end{array}\right)^{K}$, and by replicating every row $N$ times to represent the selection of all $N$ antenna elements of a certain AP, we form all possibilities for $\mathbf{Q}$. The optimization problem that performs APS using ES can be formulated as follows

$$
\begin{aligned}
& \max _{\mathbf{Q}} \min _{k} \mathrm{SINR}_{k} \\
& \text { s.t. } \sum_{j=1}^{M} q_{j, k}=S N, k=1, \ldots, K,
\end{aligned}
$$

where

$$
\mathrm{SINR}_{k}=\frac{\mathbb{E}\left[\left|A_{1}\right|^{2}\right]}{\sigma_{w}^{2}+\sum_{i=1, i \neq k}^{K} \mathbb{E}\left[\left|A_{2, i}\right|^{2}\right]+\mathbb{E}\left[\left|A_{3}\right|^{2}\right]}
$$

is the SINR of user $k, A_{1}$ is the desired signal, $\sigma_{w}^{2}$ is the noise variance, $A_{2, i}$ is the interference caused by user $i$ in user $k$, for $i \neq k, i=1, \ldots, K, A_{3}$ is the CSI error, and $q_{j, k}$ is the $j$ th element of the $k$ th column of $\mathbf{Q}$. After testing all possibilities of $\mathbf{Q}$ we choose the one that provides the highest minimum SINR.

\subsection{3}

\section{Large-Scale-Fading-Based Selection with Fixed Number of APs (LS-APS)}

As an alternative to ES-APS, we devise a LS coefficients based selection method, where user $k$ will only be associated with $S \leq L$ APs corresponding to the $S N$ largest LS coefficients for user $k$, inspired by the algorithm in [2]. Unlike the approach in [2] that chooses the number of selected APs based on their contribution to the sum of the LS coefficients, LS-APS performs APS with a fixed $S$. This allows a fair comparison with ES-APS.

To perform APS with LS-APS, as detailed in Algorithm 4, we primarily need to estimate $\boldsymbol{\beta}_{k}=\left[\beta_{1, k}, \ldots, \beta_{M, k}\right]^{T}$, for a certain user $k$, and sort the elements in a descending order. Next, we assign in an auxiliary vector $\mathbf{q}_{k}$, 1 s corresponding to the $S N$ largest elements of $\boldsymbol{\beta}_{k}$ and 0 s to the remaining entries. By grouping the $\mathbf{q}_{k}$ columns, we obtain matrix $\mathbf{Q}$. The coefficients of matrix $\mathbf{Q}$ must be applied to the matrices $\boldsymbol{\alpha}, \boldsymbol{\beta}, \hat{\mathbf{G}}$ and $\tilde{\mathbf{G}}$ and then, precoding and power allocation can be performed with these new parameters. This completes the APS stage. 
$\overline{\text { Algorithm } 4 \text { Large-Scale-Fading-Based Selection with Fixed Number of APs }}$ (LS-APS)

1: Estimate $\boldsymbol{\beta}_{k}=\left[\beta_{1, k}, \ldots, \beta_{M, k}\right]^{T}$ for user $k$ and sort the elements in a descending order.

2: Choose the largest $S N$ elements of $\boldsymbol{\beta}_{k}$.

3: In the auxiliary vector $\mathbf{q}_{k}$, assign 1 s to the corresponding $S N$ largest elements of $\boldsymbol{\beta}_{k}$ and $0 \mathrm{~s}$ to the remaining ones.

4: Let $\mathbf{q}_{k}, k=1, \ldots, K$ be the columns of the matrix $\mathbf{Q}$.

5: Let $\alpha_{m, k}^{\prime}=q_{m, k} \cdot \alpha_{m, k}, \beta_{m, k}^{\prime}=q_{m, k} \cdot \beta_{m, k}, \hat{g}_{m, k}^{\prime}=q_{m, k} \cdot \hat{g}_{m, k}$ and $\tilde{g}_{m, k}^{\prime}=q_{m, k} \cdot \tilde{g}_{m, k}$, where $q_{m, k}$ is the $m$ th element of vector $\mathbf{q}_{k}$.

6: Perform precoding and power allocation with the new parameters.

Below we give an example of vector $\boldsymbol{\beta}_{k}$ with $L=6, N=1$ and $S=3$ :

$$
\begin{gathered}
\beta_{k}=\left[\begin{array}{c}
\beta_{1, k} \\
\beta_{2, k} \\
\beta_{3, k} \\
\beta_{4, k} \\
\beta_{5, k} \\
\beta_{6, k}
\end{array}\right] \stackrel{\text { descending order }}{\longrightarrow}\left[\begin{array}{c}
\beta_{3, k} \\
\beta_{4, k} \\
\beta_{1, k} \\
\beta_{5, k} \\
\beta_{2, k} \\
\beta_{6, k}
\end{array}\right] \stackrel{\text { corresponding } q_{k}}{\longrightarrow}\left[\begin{array}{l}
1 \\
0 \\
1 \\
1 \\
0 \\
0
\end{array}\right] \\
\beta_{3, k}>\beta_{4, k}>\beta_{1, k}>\beta_{5, k}>\beta_{2, k}>\beta_{6, k}
\end{gathered}
$$

\section{2}

\section{Iterative MMSE Precoder with Total Power Constraint}

This part of the work presents the derivation of a linear MMSE precoder. The technique is based on a total power constraint and iteratively adapted to perform power allocation in order to maximize the minimum SINR with a per-antenna power constraint or to minimize the MSE under a per-antenna power constraint. All the variables referring to this technique have a subscript "MMSE".

Unlike existing approaches $[9,56]$, in this specific precoder, we consider power allocation in the derivation and take into account the CSI matrix, $\hat{\mathbf{G}}$, instead of the actual channel matrix $\mathbf{G}$, since the APs have imperfect CSI. Furthermore, we derive an iterative MMSE precoder with power allocation in order to maximize the minimum SINR. If the conventional transmit WF in [56] was applied to this type of system, the performance would be degraded due to the lack of appropriate power allocation. In the precoder design we also take into account a normalization factor $f^{-1}$ at the receivers, which can be interpreted as an automatic gain control [56]. 
By modifying the expression in (2-14) and inserting a power allocation matrix $\mathbf{N}$, the $k$ th user receives

$$
y_{k}=\sqrt{\rho_{f}} \mathbf{g}_{k}^{T} \mathbf{P} \mathbf{N} \mathbf{s}+w_{k},
$$

where $\mathbf{N} \in \mathbb{R}_{+}^{K \times K}$ is the power allocation diagonal matrix with $\sqrt{\eta_{1}}, \ldots, \sqrt{\eta_{K}}$ on its diagonal and $\eta_{k}$ is the power coefficient of user $k$. For all users combined, we have

$$
\mathbf{y}=\sqrt{\rho_{f}} \mathbf{G}^{T} \mathbf{P} \mathbf{N s}+\mathbf{w} .
$$

To obtain the MMSE precoder, the following optimization is solved [56]:

$$
\begin{aligned}
& \left\{\mathbf{P}_{\mathrm{MMSE}}, \mathbf{N}, f_{\mathrm{MMSE}}\right\}=\operatorname{argmin}_{\{\mathbf{P}, \mathbf{N}, f\}} \mathbb{E}\left[\left\|\mathbf{s}-f^{-1} \mathbf{y}\right\|_{2}^{2}\right] \\
& \text { s.t.: } \mathbb{E}\left[\|\mathbf{x}\|_{2}^{2}\right]=E_{t r},
\end{aligned}
$$

where the transmitted signal is

$$
\mathbf{x}=\sqrt{\rho_{f}} \mathbf{P} \mathbf{N} \mathbf{s}
$$

The average transmitted power is obtained through

$$
\begin{aligned}
\mathbb{E}\left[\|\mathbf{x}\|_{2}^{2}\right] & =\mathbb{E}\left[\left(\sqrt{\rho_{f}} \mathbf{P} \mathbf{N} \mathbf{s}\right)^{H}\left(\sqrt{\rho_{f}} \mathbf{P} \mathbf{N} \mathbf{s}\right)\right] \\
& =\rho_{f} \mathbb{E}\left[\mathbf{s}^{H} \mathbf{N}^{H} \mathbf{P}^{H} \mathbf{P} \mathbf{N} \mathbf{s}\right] \\
& =\rho_{f} \operatorname{tr}\left(\mathbb{E}\left[\mathbf{s}^{H} \mathbf{N}^{H} \mathbf{P}^{H} \mathbf{P N} \mathbf{s}\right]\right) \\
& =\rho_{f} \mathbb{E}\left[\operatorname{tr}\left(\mathbf{N}^{H} \mathbf{P}^{H} \mathbf{P} \mathbf{N s s ^ { H }}\right)\right] \\
& =\rho_{f} \operatorname{tr}\left(\mathbf{P} \mathbf{N C}_{s} \mathbf{N}^{H} \mathbf{P}^{H}\right)=E_{t r}
\end{aligned}
$$

where $\mathbf{C}_{s}=\mathbb{E}\left[\mathbf{s s}^{H}\right]$ is the symbol covariance matrix.

The MSE cost function is given by:

$$
\begin{aligned}
\mathbb{E}\left[\left\|\mathbf{s}-f^{-1} \mathbf{y}\right\|_{2}^{2}\right]=\mathbb{E} & {\left[\left(\mathbf{s}-f^{-1} \mathbf{y}\right)^{H}\left(\mathbf{s}-f^{-1} \mathbf{y}\right)\right] } \\
=\mathbb{E} & {\left[\mathbf{s}^{H} \mathbf{s}-f^{-1} \mathbf{s}^{H} \mathbf{y}-f^{-1} \mathbf{y}^{H} \mathbf{s}+f^{-2} \mathbf{y}^{H} \mathbf{y}\right] } \\
=\mathbb{E} & {\left[\mathbf{s}^{H} \mathbf{s}-f^{-1} \mathbf{s}^{H}\left(\sqrt{\rho_{f}} \hat{\mathbf{G}}^{T} \mathbf{P} \mathbf{N} \mathbf{s}+\mathbf{w}\right)\right.} \\
& -f^{-1}\left(\sqrt{\rho_{f}} \hat{\mathbf{G}}^{T} \mathbf{P} \mathbf{N} \mathbf{s}+\mathbf{w}\right)^{H} \mathbf{s} \\
& \left.+f^{-2}\left(\sqrt{\rho_{f}} \hat{\mathbf{G}}^{T} \mathbf{P} \mathbf{N} \mathbf{s}+\mathbf{w}\right)^{H}\left(\sqrt{\rho_{f}} \hat{\mathbf{G}}^{T} \mathbf{P N}+\mathbf{w}\right)\right]
\end{aligned}
$$




$$
\begin{aligned}
=\mathbb{E} & {\left[\mathbf{s}^{H} \mathbf{s}\right]-f^{-1} \mathbb{E}\left[\sqrt{\rho_{f}} \mathbf{s}^{H} \hat{\mathbf{G}}^{T} \mathbf{P} \mathbf{N} \mathbf{s}\right]-f^{-1} \mathbb{E}\left[\mathbf{s}^{H} \mathbf{w}\right] } \\
& -f^{-1} \mathbb{E}\left[\sqrt{\rho_{f}} \mathbf{s}^{H} \mathbf{N}^{H} \mathbf{P}^{H} \hat{\mathbf{G}}^{*} \mathbf{s}\right]-f^{-1} \mathbb{E}\left[\mathbf{w}^{H} \mathbf{s}\right] \\
& +f^{-2} \mathbb{E}\left[\rho_{f} \mathbf{s}^{H} \mathbf{N}^{H} \mathbf{P}^{H} \hat{\mathbf{G}}^{*} \hat{\mathbf{G}}^{T} \mathbf{P} \mathbf{N} \mathbf{s}\right] \\
& +f^{-2} \mathbb{E}\left[\sqrt{\rho_{f}} \mathbf{s}^{H} \mathbf{N}^{H} \mathbf{P}^{H} \hat{\mathbf{G}}^{*} \mathbf{w}\right]+f^{-2} \mathbb{E}\left[\sqrt{\rho_{f}} \mathbf{w}^{H} \hat{\mathbf{G}}^{T} \mathbf{P N} \mathbf{s}\right] \\
& +f^{-2} \mathbb{E}\left[\mathbf{w}^{H} \mathbf{w}\right] .
\end{aligned}
$$

Data symbols are independent from the noise, leading to:

$$
\begin{aligned}
\mathbb{E}\left[\left\|\mathbf{s}-f^{-1} \mathbf{y}\right\|_{2}^{2}\right]=\mathbb{E} & {\left[\mathbf{s}^{H} \mathbf{s}\right]-f^{-1} \sqrt{\rho_{f}} \mathbb{E}\left[\mathbf{s}^{H} \hat{\mathbf{G}}^{T} \mathbf{P} \mathbf{N} \mathbf{s}\right] } \\
& -f^{-1} \sqrt{\rho_{f}} \mathbb{E}\left[\mathbf{s}^{H} \mathbf{N}^{H} \mathbf{P}^{H} \hat{\mathbf{G}}^{*} \mathbf{s}\right] \\
& +f^{-2} \rho_{f} \mathbb{E}\left[\mathbf{s}^{H} \mathbf{N}^{H} \mathbf{P}^{H} \hat{\mathbf{G}}^{*} \hat{\mathbf{G}}^{T} \mathbf{P} \mathbf{N} \mathbf{s}\right]+f^{-2} \mathbb{E}\left[\mathbf{w}^{H} \mathbf{w}\right]
\end{aligned}
$$

Applying the trace operator we have:

$$
\begin{aligned}
\mathbb{E}\left[\left\|\mathbf{s}-f^{-1} \mathbf{y}\right\|_{2}^{2}\right]=\operatorname{tr} & \left(\mathbb{E}\left[\mathbf{s}^{H} \mathbf{s}\right]\right)-f^{-1} \sqrt{\rho_{f}} \operatorname{tr}\left(\mathbb{E}\left[\mathbf{s}^{H} \hat{\mathbf{G}}^{T} \mathbf{P} \mathbf{N} \mathbf{s}\right]\right) \\
& -f^{-1} \sqrt{\rho_{f}} \operatorname{tr}\left(\mathbb{E}\left[\mathbf{s}^{H} \mathbf{N}^{H} \mathbf{P}^{H} \hat{\mathbf{G}}^{*} \mathbf{s}\right]\right) \\
& +f^{-2} \rho_{f} \operatorname{tr}\left(\mathbb{E}\left[\mathbf{s}^{H} \mathbf{N}^{H} \mathbf{P}^{H} \hat{\mathbf{G}}^{*} \hat{\mathbf{G}}^{T} \mathbf{P N \mathbf { N }}\right]\right)+f^{-2} \operatorname{tr}\left(\mathbb{E}\left[\mathbf{w}^{H} \mathbf{w}\right]\right) \\
=\mathbb{E} & \left.\operatorname{tr}\left(\mathbf{s s}^{H}\right)\right]-f^{-1} \sqrt{\rho_{f}} \mathbb{E}\left[\operatorname{tr}\left(\hat{\mathbf{G}}^{T} \mathbf{P} \mathbf{N} \mathbf{s s}^{H}\right)\right] \\
& -f^{-1} \sqrt{\rho_{f}} \mathbb{E}\left[\operatorname{tr}\left(\mathbf{N}^{H} \mathbf{P}^{H} \hat{\mathbf{G}}^{*} \mathbf{s s}^{H}\right)\right] \\
& +f^{-2} \rho_{f} \mathbb{E}\left[\operatorname{tr}\left(\mathbf{N}^{H} \mathbf{P}^{H} \hat{\mathbf{G}}^{*} \hat{\mathbf{G}}^{T} \mathbf{P} \mathbf{N} \mathbf{s} \mathbf{s}^{H}\right)\right]+f^{-2} \mathbb{E}\left[\operatorname{tr}\left(\mathbf{w} \mathbf{w}^{H}\right)\right] \\
= & \operatorname{tr}\left(\mathbf{C}_{s}\right)-f^{-1} \sqrt{\rho_{f}} \operatorname{tr}\left(\hat{\mathbf{G}}^{T} \mathbf{P N C}_{s}\right) \\
& -f^{-1} \sqrt{\rho_{f}} \operatorname{tr}\left(\mathbf{N}^{H} \mathbf{P}^{H} \hat{\mathbf{G}}^{*} \mathbf{C}_{s}\right) \\
& +f^{-2} \rho_{f} \operatorname{tr}\left(\mathbf{N}^{H} \mathbf{P}^{H} \hat{\mathbf{G}}^{*} \hat{\mathbf{G}}^{T} \mathbf{P} \mathbf{N} \mathbf{C}_{s}\right)+f^{-2} \operatorname{tr}\left(\mathbf{C}_{w}\right)
\end{aligned}
$$

where $\mathbf{C}_{w}=\mathbb{E}\left[\mathbf{w w}^{H}\right]$ is the noise covariance matrix.

By constructing the Lagrangian function with the Lagrange multiplier $\lambda$, setting its derivatives to zero and bearing in mind a power allocation matrix $\mathbf{N}$, we can compute the precoder $\mathbf{P}$ and the normalization $f$, as shown below: 
Chapter 3. Iterative AP Selection, MMSE Precoding and Power Allocation for

$$
\begin{aligned}
& \mathcal{L}(\mathbf{P}, \mathbf{N}, f, \lambda)=\mathbb{E}\left[\left\|\mathbf{s}-f^{-1} \mathbf{y}\right\|_{2}^{2}\right]+\lambda\left(\rho_{f} \operatorname{tr}\left(\mathbf{P} \mathbf{N C}_{s} \mathbf{N}^{H} \mathbf{P}^{H}\right)-E_{t r}\right) \\
&=\operatorname{tr}\left(\mathbf{C}_{s}\right)-f^{-1} \sqrt{\rho_{f}} \operatorname{tr}\left(\hat{\mathbf{G}}^{T} \mathbf{P} \mathbf{N} \mathbf{C}_{s}\right) \\
&-f^{-1} \sqrt{\rho_{f}} \operatorname{tr}\left(\hat{\mathbf{G}}^{*} \mathbf{C}_{s} \mathbf{N}^{H} \mathbf{P}^{H}\right) \\
&+f^{-2} \rho_{f} \operatorname{tr}\left(\hat{\mathbf{G}}^{*} \hat{\mathbf{G}}^{T} \mathbf{P} \mathbf{N} \mathbf{C}_{s} \mathbf{N}^{H} \mathbf{P}^{H}\right)+f^{-2} \operatorname{tr}\left(\mathbf{C}_{w}\right) \\
&+\lambda\left(\rho_{f} \operatorname{tr}\left(\mathbf{P} \mathbf{N C}_{s} \mathbf{N}^{H} \mathbf{P}^{H}\right)-E_{t r}\right) .
\end{aligned}
$$

Using Wirtinger's calculus and the result of the partial derivative $\partial \operatorname{tr}\left(\mathbf{B X}^{H}\right) / \partial \mathbf{X}^{*}=\mathbf{B}$, we have

$$
\begin{gathered}
\frac{\partial \mathcal{L}(\mathbf{P}, \mathbf{N}, f, \lambda)}{\partial \mathbf{P}^{*}}=f^{-2} \rho_{f} \hat{\mathbf{G}}^{*} \hat{\mathbf{G}}^{T} \mathbf{P} \mathbf{N C}_{s} \mathbf{N}^{H}+\lambda \rho_{f} \mathbf{P} \mathbf{N C} \mathbf{C}_{s} \mathbf{N}^{H} \\
-f^{-1} \sqrt{\rho_{f}} \hat{\mathbf{G}}^{*} \mathbf{C}_{s} \mathbf{N}^{H}=0
\end{gathered}
$$

and

$$
\begin{aligned}
\frac{\partial \mathcal{L}(\mathbf{P}, \mathbf{N}, f, \lambda)}{\partial f}=f^{-2} & \sqrt{\rho_{f}} \operatorname{tr}\left(\hat{\mathbf{G}}^{T} \mathbf{P} \mathbf{N} \mathbf{C}_{s}\right)+f^{-2} \sqrt{\rho_{f}} \operatorname{tr}\left(\mathbf{N}^{H} \mathbf{P}^{H} \hat{\mathbf{G}}^{*} \mathbf{C}_{s}\right) \\
& -2 f^{-3} \rho_{f} \operatorname{tr}\left(\mathbf{N}^{H} \mathbf{P}^{H} \hat{\mathbf{G}}^{*} \hat{\mathbf{G}}^{T} \mathbf{P} \mathbf{N} \mathbf{C}_{s}\right)-2 f^{-3} \operatorname{tr}\left(\mathbf{C}_{w}\right)=0
\end{aligned}
$$

By solving (3-15),

$$
\begin{aligned}
& f^{-2} \rho_{f} \hat{\mathbf{G}}^{*} \hat{\mathbf{G}}^{T} \mathbf{P} \mathbf{N C}_{s} \mathbf{N}^{H}+\lambda \rho_{f} \mathbf{P} \mathbf{N C}_{s} \mathbf{N}^{H}-f^{-1} \sqrt{\rho_{f}} \hat{\mathbf{G}}^{*} \mathbf{C}_{s} \mathbf{N}^{H}=0 \\
& f \sqrt{\rho_{f}} \hat{\mathbf{G}}^{*} \mathbf{C}_{s} \mathbf{N}^{H}=\rho_{f} \hat{\mathbf{G}}^{*} \hat{\mathbf{G}}^{T} \mathbf{P} \mathbf{N C}_{s} \mathbf{N}^{H}+\lambda f^{2} \rho_{f} \mathbf{P} \mathbf{N C}_{s} \mathbf{N}^{H} \\
& f \sqrt{\rho_{f}} \hat{\mathbf{G}}^{*}=\rho_{f} \hat{\mathbf{G}}^{*} \hat{\mathbf{G}}^{T} \mathbf{P} \mathbf{N}+\lambda f^{2} \rho_{f} \mathbf{P} \mathbf{N} \\
& f \sqrt{\rho_{f}} \hat{\mathbf{G}}^{*}=\rho_{f}\left(\hat{\mathbf{G}}^{*} \hat{\mathbf{G}}^{T}+\lambda f^{2} \mathbf{I}_{M}\right) \mathbf{P} \mathbf{N} \\
& \mathbf{P} \mathbf{N}=\frac{f}{\sqrt{\rho_{f}}} \underbrace{\left(\hat{\mathbf{G}}^{*} \hat{\mathbf{G}}^{T}+\lambda f^{2} \mathbf{I}_{M}\right)^{-1} \hat{\mathbf{G}}^{*}}_{\tilde{\mathbf{P}}} \\
& \mathbf{P}=\frac{f}{\sqrt{\rho_{f}}} \underbrace{\left(\hat{\mathbf{G}}^{*} \hat{\mathbf{G}}^{T}+\lambda f^{2} \mathbf{I}_{M}\right)^{-1} \hat{\mathbf{G}}^{*} \mathbf{N}^{-1}}_{\tilde{\mathbf{P}}} \\
& \mathbf{P}=\frac{f}{\sqrt{\rho_{f}}} \tilde{\mathbf{P}} \mathbf{N}^{-1} .
\end{aligned}
$$

By using the expression in (3-16), we arrive at 
Chapter 3. Iterative AP Selection, MMSE Precoding and Power Allocation for

$$
\begin{aligned}
& f^{-2} \sqrt{\rho_{f}} \operatorname{tr}\left(\hat{\mathbf{G}}^{T} \mathbf{P} \mathbf{N} \mathbf{C}_{s}\right)+f^{-2} \sqrt{\rho_{f}} \operatorname{tr}\left(\mathbf{N}^{H} \mathbf{P}^{H} \hat{\mathbf{G}}^{*} \mathbf{C}_{s}\right) \\
& -2 f^{-3} \rho_{f} \operatorname{tr}\left(\mathbf{N}^{H} \mathbf{P}^{H} \hat{\mathbf{G}}^{*} \hat{\mathbf{G}}^{T} \mathbf{P} \mathbf{N} \mathbf{C}_{s}\right)-2 f^{-3} \operatorname{tr}\left(\mathbf{C}_{w}\right)=0 \\
& 2 f^{-2} \sqrt{\rho_{f}} \operatorname{Re}\left(\operatorname{tr}\left(\hat{\mathbf{G}}^{T} \mathbf{P} \mathbf{N C}_{s}\right)\right)-2 f^{-3} \rho_{f} \operatorname{tr}\left(\mathbf{N}^{H} \mathbf{P}^{H} \hat{\mathbf{G}}^{*} \hat{\mathbf{G}}^{T} \mathbf{P} \mathbf{N C}_{s}\right) \\
& -2 f^{-3} \operatorname{tr}\left(\mathbf{C}_{w}\right)=0 \\
& \operatorname{tr}\left(-\left(\rho_{f} \mathbf{N}^{H} \mathbf{P}^{H} \hat{\mathbf{G}}^{*} \hat{\mathbf{G}}^{T} \mathbf{P} \mathbf{N C} \mathbf{C}_{s}+\mathbf{C}_{w}\right)+f \sqrt{\rho_{f}} \operatorname{Re}\left(\hat{\mathbf{G}}^{T} \mathbf{P} \mathbf{N} \mathbf{C}_{s}\right)\right)=0 \\
& \operatorname{tr}\left(-\left(\rho_{f} \frac{f}{\sqrt{\rho_{f}}} \mathbf{N}^{H}\left(\mathbf{N}^{-1}\right)^{H} \tilde{\mathbf{P}}^{H} \hat{\mathbf{G}}^{*} \hat{\mathbf{G}}^{T} \frac{f}{\sqrt{\rho_{f}}} \tilde{\mathbf{P}} \mathbf{N}^{-1} \mathbf{N C}_{s}+\mathbf{C}_{w}\right)\right. \\
& \left.+f \sqrt{\rho_{f}} \operatorname{Re}\left(\hat{\mathbf{G}}^{T} \frac{f}{\sqrt{\rho_{f}}} \tilde{\mathbf{P}} \mathbf{N}^{-1} \mathbf{N C}_{s}\right)\right)=0 \\
& \operatorname{tr}\left(-\left(f^{2} \tilde{\mathbf{P}}^{H} \hat{\mathbf{G}}^{*} \hat{\mathbf{G}}^{T} \tilde{\mathbf{P}} \mathbf{C}_{s}+\mathbf{C}_{w}\right)+f^{2} \operatorname{Re}\left(\hat{\mathbf{G}}^{T} \tilde{\mathbf{P}} \mathbf{C}_{s}\right)\right)=0 \\
& f^{2} \operatorname{tr}\left(\operatorname{Re}\left(\hat{\mathbf{G}}^{T} \tilde{\mathbf{P}} \mathbf{C}_{s}\right)\right)=f^{2} \operatorname{tr}\left(\hat{\mathbf{G}}^{*} \hat{\mathbf{G}}^{T} \tilde{\mathbf{P}} \mathbf{C}_{s} \tilde{\mathbf{P}}^{H}\right)+\operatorname{tr}\left(\mathbf{C}_{w}\right)
\end{aligned}
$$

Using the relation introduced in [56],

$$
\operatorname{tr}\left(\operatorname{Re}\left(\hat{\mathbf{G}}^{T} \tilde{\mathbf{P}} \mathbf{C}_{s}\right)\right)=\operatorname{tr}\left(\left(\hat{\mathbf{G}}^{*} \hat{\mathbf{G}}^{T}+\epsilon \mathbf{I}_{M}\right) \tilde{\mathbf{P}} \mathbf{C}_{s} \tilde{\mathbf{P}}^{H}\right),
$$

equalling to (3-18) and setting $\epsilon=\lambda f^{2}$, we yield

$$
\begin{aligned}
& f^{2} \operatorname{tr}\left(\left(\hat{\mathbf{G}}^{*} \hat{\mathbf{G}}^{T}+\epsilon \mathbf{I}_{M}\right) \tilde{\mathbf{P}} \mathbf{C}_{s} \tilde{\mathbf{P}}^{H}\right)=f^{2} \operatorname{tr}\left(\hat{\mathbf{G}}^{*} \hat{\mathbf{G}}^{T} \tilde{\mathbf{P}} \mathbf{C}_{s} \tilde{\mathbf{P}}^{H}\right)+\operatorname{tr}\left(\mathbf{C}_{w}\right) \\
& f^{2} \operatorname{tr}\left(\hat{\mathbf{G}}^{*} \hat{\mathbf{G}}^{T} \tilde{\mathbf{P}} \mathbf{C}_{s} \tilde{\mathbf{P}}^{H}\right)+f^{2} \operatorname{tr}\left(\epsilon \tilde{\mathbf{P}} \mathbf{C}_{s} \tilde{\mathbf{P}}^{H}\right)=f^{2} \operatorname{tr}\left(\hat{\mathbf{G}}^{*} \hat{\mathbf{G}}^{T} \tilde{\mathbf{P}} \mathbf{C}_{s} \tilde{\mathbf{P}}^{H}\right)+\operatorname{tr}\left(\mathbf{C}_{w}\right) \\
& f^{2} \operatorname{tr}\left(\epsilon \tilde{\mathbf{P}} \mathbf{C}_{s} \tilde{\mathbf{P}}^{H}\right)=\operatorname{tr}\left(\mathbf{C}_{w}\right) \\
& \epsilon f^{2} \operatorname{tr}\left(\tilde{\mathbf{P}} \mathbf{C}_{s} \tilde{\mathbf{P}}^{H}\right)=\operatorname{tr}\left(\mathbf{C}_{w}\right) \\
& \epsilon E_{t r}=\operatorname{tr}\left(\mathbf{C}_{w}\right) \\
& \epsilon=\frac{\operatorname{tr}\left(\mathbf{C}_{w}\right)}{E_{t r}}
\end{aligned}
$$

since

$$
\begin{aligned}
E_{t r} & =\rho_{f} \operatorname{tr}\left(\mathbf{P} \mathbf{N C} \mathbf{N}_{s} \mathbf{N}^{H} \mathbf{P}^{H}\right) \\
& =\rho_{f} \operatorname{tr}\left(\frac{f}{\sqrt{\rho_{f}}} \tilde{\mathbf{P}} \mathbf{N}^{-1} \mathbf{N C}_{s} \mathbf{N}^{H}\left(\mathbf{N}^{-1}\right)^{H} \tilde{\mathbf{P}}^{H} \frac{f}{\sqrt{\rho_{f}}}\right) \\
& =f^{2} \operatorname{tr}\left(\tilde{\mathbf{P}} \mathbf{C}_{s} \tilde{\mathbf{P}}^{H}\right) .
\end{aligned}
$$

As a result, the MMSE precoder that takes into account power allocation for cell-free systems is written as

$$
\mathbf{P}_{\mathrm{MMSE}}=\frac{f_{\mathrm{MMSE}}}{\sqrt{\rho_{f}}}\left(\hat{\mathbf{G}}^{*} \hat{\mathbf{G}}^{T}+\frac{\operatorname{tr}\left(\mathbf{C}_{w}\right)}{E_{t r}} \mathbf{I}_{M}\right)^{-1} \hat{\mathbf{G}}^{*} \mathbf{N}^{-1},
$$

where 


$$
f_{\mathrm{MMSE}}=\sqrt{\frac{E_{t r}}{\operatorname{tr}\left(\tilde{\mathbf{P}} \mathbf{C}_{s} \tilde{\mathbf{P}}^{H}\right)}},
$$

$[\hat{\mathbf{G}}]_{m, k}=\hat{g}_{m, k}$ is the CSI matrix. The noise is uncorrelated meaning that $\mathbf{C}_{w}=\sigma_{w}^{2} \mathbf{I}_{K}$ and $\operatorname{tr}\left(\mathbf{C}_{w}\right)=K \sigma_{w}^{2}$. We initialize the precoder considering a power allocation matrix $\mathbf{N}=\mathbf{I}_{K}$. After the MMSE precoder is obtained, we perform power allocation. With the new power allocation matrix $\mathbf{N}$, we substitute it in $\mathbf{P}_{\text {MMSE }}$. The last step of the iteration is to recalculate matrix $\mathbf{N}$ by employing $\mathbf{P}_{\text {MMSE }}$ to perform power allocation. This final $\mathbf{N}$, called $\mathbf{N}_{\text {MMSE }}$, guarantees that the power constraint is satisfied. Note that the $\mathbf{N}$ present in the $\mathbf{P}_{\text {MMSE }}$ expression is different from the final $\mathbf{N}_{\mathrm{MMSE}}$. Consequently, $\mathbf{N}$ and $\mathbf{N}_{\mathrm{MMSE}}$ will not cancel each other.

\section{3}

\section{Power Allocation}

We develop OPA, APA and UPA techniques to calculate $\mathbf{N}$, a diagonal matrix with $\sqrt{\eta_{1}}, \ldots, \sqrt{\eta_{K}}$ on its main diagonal, which is used to recompute the precoder $\mathbf{P}_{\mathrm{MMSE}}$ and find the power allocation matrix $\mathbf{N}_{\mathrm{MMSE}}$.

\subsection{1}

\section{Optimal Power Allocation (OPA)}

Before presenting the OPA algorithm, we need to derive the per-antenna power constraint, taking into consideration a power allocation matrix. Given a matrix $\mathbf{A}=\mathbf{P}_{\text {MMSE }} \mathbf{N}_{\text {MMSE }}$, the transmitted power for the $m$ th antenna can be expressed as

$$
\begin{aligned}
\rho_{f} \mathbb{E}\left[\mathbf{a}_{m}^{T} \mathbf{a}_{m}^{*}\right] & =\rho_{f} \mathbb{E}\left[\mathbf{N}_{\mathrm{MMSE}} \mathbf{p}_{m}^{T} \mathbf{p}_{m}^{*} \mathbf{N}_{\mathrm{MMSE}}\right] \\
& =\rho_{f} \operatorname{tr}\left(\mathbb{E}\left[\mathbf{N}_{\mathrm{MMSE}} \mathbf{p}_{m}^{T} \mathbf{p}_{m}^{*} \mathbf{N}_{\mathrm{MMSE}}\right]\right) \\
& =\rho_{f} \operatorname{tr}\left(\mathbf{N}_{\mathrm{MMSE}}^{2} \mathbb{E}\left[\mathbf{p}_{m}^{T} \mathbf{p}_{m}^{*}\right]\right) \\
& =\rho_{f} \sum_{i=1}^{K} \eta_{i} \delta_{m, i}
\end{aligned}
$$

where

$$
\boldsymbol{\delta}_{m}=\operatorname{diag}\left\{\mathbb{E}\left[\mathbf{p}_{m}^{T} \mathbf{p}_{m}^{*}\right]\right\}, m=1, \ldots, M,
$$

$\mathbf{a}_{m}$ is the $m$ th row of matrix $\mathbf{A}, \mathbf{p}_{m}=\left[p_{m, 1}, \ldots, p_{m, K}\right]$ is the $m$ th row of the precoder $\mathbf{P}_{\text {MMSE }}$ and $\delta_{m, i}$ is the $i$ th element of vector $\boldsymbol{\delta}_{m}$. The maximum transmitted power per-antenna is $\rho_{f}$, resulting in the following per-antenna power constraint 


$$
\begin{gathered}
\rho_{f} \sum_{i=1}^{K} \eta_{i} \delta_{m, i} \leq \rho_{f}, m=1, \ldots, M \\
\sum_{i=1}^{K} \eta_{i} \delta_{m, i} \leq 1, m=1, \ldots, M
\end{gathered}
$$

Therefore, the max-min fairness power allocation problem with antenna power constraint becomes

$$
\begin{aligned}
& \max _{\boldsymbol{\eta}} \min _{k} \operatorname{SINR}_{k}(\boldsymbol{\eta}) \\
& \text { s.t. } \sum_{i=1}^{K} \eta_{i} \delta_{m, i} \leq 1, m=1, \ldots, M,
\end{aligned}
$$

where

$$
\operatorname{SINR}_{k}=\frac{\mathbb{E}\left[\left|A_{1}\right|^{2}\right]}{\sigma_{w}^{2}+\sum_{i=1, i \neq k}^{K} \mathbb{E}\left[\left|A_{2, i}\right|^{2}\right]+\mathbb{E}\left[\left|A_{3}\right|^{2}\right]}
$$

is the SINR of user $k, A_{1}$ is the desired signal, $\sigma_{w}^{2}$ is the noise variance, $A_{2, i}$ is the interference caused by user $i$ in user $k$, for $i \neq k, i=1, \ldots, K$ and $A_{3}$ is the CSI error.

The optimization problem in an epigraph form employs the bisection method at each step and is formulated by

$$
\begin{aligned}
& \text { find } \boldsymbol{\eta} \\
& \text { s.t. } \operatorname{SINR}_{\mathrm{k}}(\boldsymbol{\eta}) \geq t, k=1, \ldots, K, \\
& \qquad \sum_{i=1}^{K} \eta_{i} \delta_{m, i} \leq 1, m=1, \ldots, M,
\end{aligned}
$$

where $t=\frac{t_{b}+t_{e}}{2}$ is the midpoint of a chosen interval $\left(t_{b}, t_{e}\right)$ that contains the optimal value $t^{*}$, as in [1]. The parameter $T_{\mathrm{OPA}}$ refers to the number of iterations for the bisection method. Its procedure is detailed in Algorithm 1.

\subsection{2}

\section{Adaptive Power Allocation (APA)}

We propose an adaptive SG learning algorithm, which takes into account the gradient of the error in (3-13) to perform APA and has a per-antenna power constraint. Our main objective here is to propose alternatives to the OPA and UPA algorithms.

The APA technique aims to adjust the power coefficients $\eta_{k}$ so that they minimize the effect of the interference at the received signal vector $\mathbf{y},[59,60]$. Firstly, an unconstrained optimization is performed in accordance with (3-32) to minimize the interference. Second, a per-antenna power constraint is applied in order to fulfil the cell-free network power requirements. 
To start with the optimization we will first calculate the cost function as:

$$
\begin{aligned}
\mathcal{C}(\mathbf{N})=\mathbb{E} & {\left[\left\|\mathbf{s}-f^{-1} \mathbf{y}\right\|_{2}^{2}\right]=\operatorname{tr}\left(\mathbf{C}_{s}\right)-f^{-1} \sqrt{\rho_{f}} \operatorname{tr}\left(\hat{\mathbf{G}}^{T} \mathbf{P} \mathbf{N} \mathbf{C}_{\mathbf{s}}\right) } \\
& -f^{-1} \sqrt{\rho_{f}} \operatorname{tr}\left(\mathbf{P}^{H} \hat{\mathbf{G}}^{*} \mathbf{C}_{s} \mathbf{N}^{H}\right)+f^{-2} \rho_{f} \operatorname{tr}\left(\mathbf{P}^{H} \hat{\mathbf{G}}^{*}\right. \\
& \left.\hat{\mathbf{G}}^{T} \mathbf{P} \mathbf{N C}_{s} \mathbf{N}^{H}\right)+f^{-2} \operatorname{tr}\left(\mathbf{C}_{w}\right)
\end{aligned}
$$

The instantaneous gradient with respect to $\mathbf{N}^{*}$ is

$$
\underset{\mathbf{N}^{*}}{\hat{\nabla} \mathcal{C}}(\mathbf{N})=-f^{-1} \sqrt{\rho_{f}} \mathbf{P}^{H} \hat{\mathbf{G}}^{*} \mathbf{C}_{s}+f^{-2} \rho_{f} \mathbf{P}^{H} \hat{\mathbf{G}}^{*} \hat{\mathbf{G}}^{T} \mathbf{P} \mathbf{N} \mathbf{C}_{s}
$$

Hence, the matrix $\mathbf{N}$ is updated by

$$
\begin{array}{r}
\mathbf{N}[i+1]=\mathbf{N}[i]-\mu\left(-f^{-1} \sqrt{\rho_{f}} \mathbf{P}^{H} \hat{\mathbf{G}}^{*} \mathbf{C}_{s}\right. \\
\left.+f^{-2} \rho_{f} \mathbf{P}^{H} \hat{\mathbf{G}}^{*} \hat{\mathbf{G}}^{T} \mathbf{P} \mathbf{N}[i] \mathbf{C}_{s}\right)
\end{array}
$$

In Algorithm 5, the adaptive SG learning strategy that performs APA is explained in details:

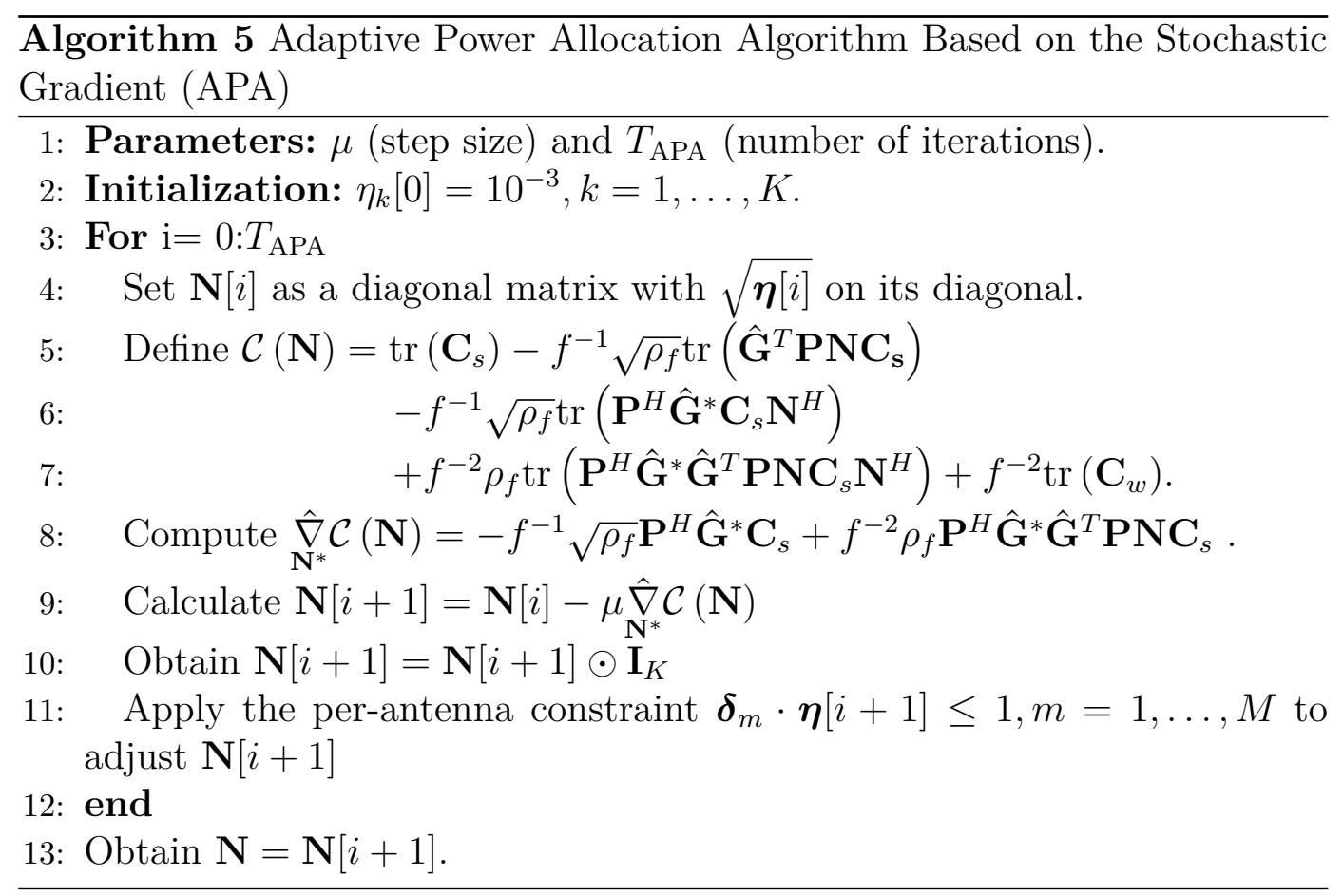

The SG power allocation algorithm for APA has a complexity of $\mathcal{O}\left(T_{\mathrm{APA}} M K^{2}\right)$ and converges within only a few iterations. In Fig. 3.1 we illustrate the performance of the APA algorithm using a step size of $\mu=0.25$ that converges within $T_{\mathrm{APA}}=5$ iterations. 


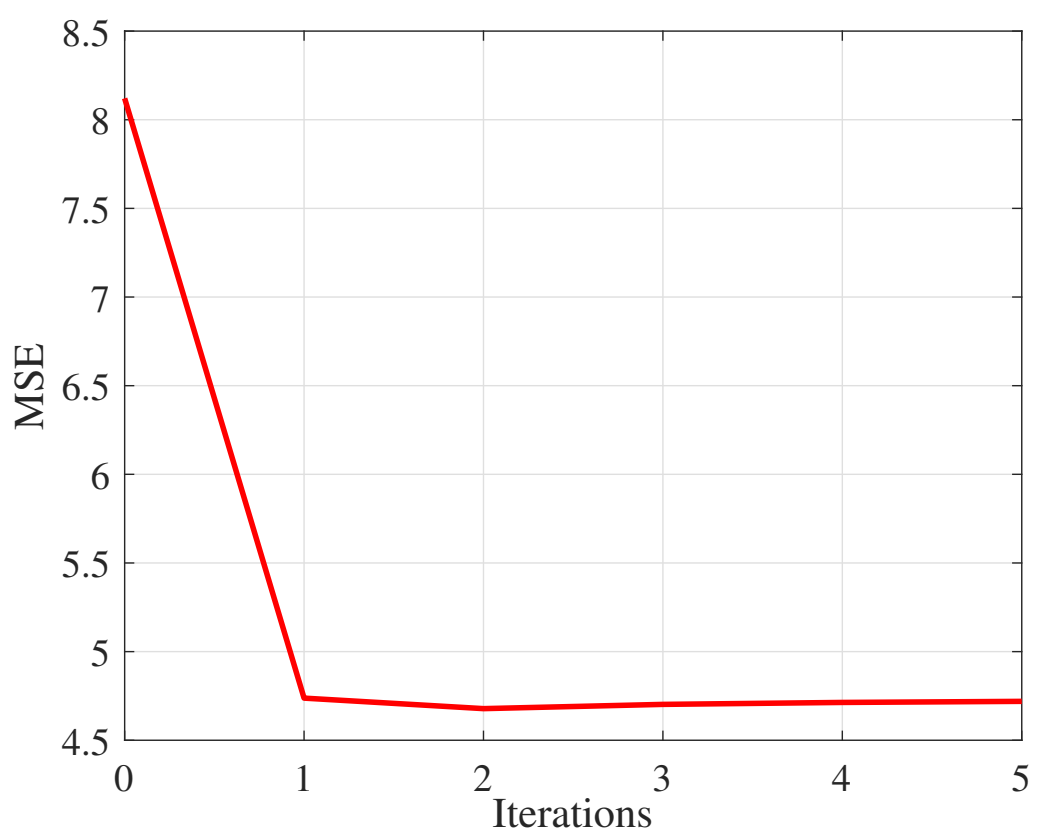

Figure 3.1: MSE vs. Iterations with $L=24, N=4, S=12, K=8, n=1$, $\mathrm{SNR}=25 \mathrm{~dB}, \mu=0.25$ and $E_{t r}=M \rho_{f}$.

\subsection{3}

\section{Uniform Power Allocation (UPA)}

As an alternative to APA, an UPA scheme is proposed, based on the one in [9] where a per-antenna power constraint is taken into account. Think of a case where we wish to find equal $\eta_{k}$ and a certain antenna element $m$ that transmits with full power. Looking for an $\eta_{k}$ at its minimum possible value, we have

$$
\eta_{k}=1 /\left(\max _{m} \sum_{i=1}^{K} \delta_{m, i}\right), k=1, \ldots, K
$$

where $\delta_{m, i}$ is the $i$ th element of vector $\boldsymbol{\delta}_{m}$. Although (3-33) is a suboptimal solution, it has low complexity, is simple and has the potential to show the benefits of the MMSE precoder.

\section{4}

\section{Analysis}

In this section, we develop a sum-rate analysis of the studied techniques along with the computational complexity of the proposed and existing algorithms. 


\subsection{1}

\section{Sum-Rate}

First, we will expand expressions (3-6) and (3-22) to obtain the received signal by user $k$ considering the MMSE precoder with total power constraint:

$$
\begin{aligned}
& y_{k}= \sqrt{\rho_{f}} \mathbf{g}_{k}^{T} \mathbf{P}_{\text {MMSE }} \mathbf{N}_{\text {MMSE }} \mathbf{s}+w_{k} \\
&= \sqrt{\rho_{f}}\left(\hat{\mathbf{g}}_{k}+\tilde{\mathbf{g}}_{k}\right)^{T} \frac{f_{\mathrm{MMSE}}}{\sqrt{\rho_{f}}}\left(\hat{\mathbf{G}}^{*} \hat{\mathbf{G}}^{T}+\frac{K \sigma_{w}^{2}}{E_{t r}} \mathbf{I}_{M}\right)^{-1} \hat{\mathbf{G}}^{*} \mathbf{N}^{-1} \mathbf{N}_{\mathrm{MMSE}} \mathbf{s}+w_{k} \\
&= \underbrace{\sqrt{\rho_{f}} \hat{\mathbf{g}}_{k}^{T} \frac{f_{\mathrm{MMSE}}}{\sqrt{\rho_{f}}}\left(\hat{\mathbf{G}}^{*} \hat{\mathbf{G}}^{T}+\frac{K \sigma_{w}^{2}}{E_{t r}} \mathbf{I}_{M}\right)^{-1} \hat{\mathbf{G}}^{*} \mathbf{N}^{-1} \mathbf{N}_{\mathrm{MMSE}} \mathbf{s}}_{\text {desired signal }+ \text { interference }}+ \\
& \underbrace{\sqrt{\rho_{f}} \tilde{\mathbf{g}}_{k}^{T} \frac{f_{\mathrm{MMSE}}}{\sqrt{\rho_{f}}}\left(\hat{\mathbf{G}}^{*} \hat{\mathbf{G}}^{T}+\frac{K \sigma_{w}^{2}}{E_{t r}} \mathbf{I}_{M}\right)^{-1} \hat{\mathbf{G}}^{*} \mathbf{N}^{-1} \mathbf{N}_{\text {MMSE }}}_{\text {CSI error }} \mathbf{S}+w_{k},
\end{aligned}
$$

where $\hat{\mathbf{g}}_{k}=\left[\hat{g}_{1, k}, \ldots, \hat{g}_{M, k}\right]^{T}$ is the CSI vector for user $k$ and $\tilde{\mathbf{g}}_{k}=$ $\left[\tilde{g}_{1, k}, \ldots, \tilde{g}_{M, k}\right]^{T}$ is the CSI error vector for user $k$.

Assuming Gaussian signalling, the achievable rate of user $k$ with the iterative MMSE precoder is given by

$$
R_{k, \mathrm{MMSE}}=\log _{2}\left(1+\mathrm{SINR}_{k, \mathrm{MMSE}}\right) .
$$

The sum-rate is described as

$$
R_{\mathrm{MMSE}}=\sum_{k=1}^{K} \log _{2}\left(1+\mathrm{SINR}_{k, \mathrm{MMSE}}\right),
$$

where

$$
\operatorname{SINR}_{k, \mathrm{MMSE}}=\frac{\mathbb{E}\left[\left|A_{1}\right|^{2}\right]}{\sigma_{w}^{2}+\sum_{i=1, i \neq k}^{K} \mathbb{E}\left[\left|A_{2, i}\right|^{2}\right]+\mathbb{E}\left[\left|A_{3}\right|^{2}\right]}
$$

In the expression above, the quantity

$$
A_{1}=\sqrt{\rho_{f}} \hat{\mathbf{g}}_{k}^{T} \mathbf{p}_{k} \sqrt{\eta_{k}} s_{k}
$$

is the desired signal, the parameter

$$
A_{2, i}=\sqrt{\rho_{f}} \hat{\mathbf{g}}_{k}^{T} \mathbf{p}_{i} \sqrt{\eta_{i}} s_{i}, \text { for } i \neq k, i=1, \ldots, K,
$$

is the interference caused by user $i$ in user $k$ and

$$
A_{3}=\sqrt{\rho_{f}} \tilde{\mathbf{g}}_{k}^{T} \mathbf{P}_{\mathrm{MMSE}} \mathbf{N}_{\mathrm{MMSE}} \mathbf{S}
$$

refers to CSI error.

The mean-square values of $A_{1}, A_{2, i}$ and $A_{3}$ are computed as follows: 


$$
\begin{aligned}
\mathbb{E}\left[\left|A_{1}\right|^{2}\right] & =\mathbb{E}\left[\left(\sqrt{\rho_{f}} \hat{\mathbf{g}}_{k}^{T} \mathbf{p}_{k} \sqrt{\eta_{k}} s_{k}\right)^{H}\left(\sqrt{\rho_{f}} \hat{\mathbf{g}}_{k}^{T} \mathbf{p}_{k} \sqrt{\eta_{k}} s_{k}\right)\right] \\
= & \mathbb{E}\left[\sqrt{\rho_{f}} s_{k}^{*} \sqrt{\eta_{k}} \mathbf{p}_{k}^{H} \hat{\mathbf{g}}_{k}^{*} \sqrt{\rho_{f}} \hat{\mathbf{g}}_{k}^{T} \mathbf{p}_{k} \sqrt{\eta_{k}} s_{k}\right] \\
= & \rho_{f} \operatorname{tr}\left(\mathbb{E}\left[\sqrt{\eta_{k}} \mathbf{p}_{k}^{H} \hat{\mathbf{g}}_{k}^{*} \hat{\mathbf{g}}_{k}^{T} \mathbf{p}_{k} \sqrt{\eta_{k}} s_{k} s_{k}^{*}\right]\right) \\
= & \rho_{f} \operatorname{tr}\left(\eta_{k} \mathbb{E}\left[\mathbf{p}_{k}^{H} \hat{\mathbf{g}}_{k}^{*} \hat{\mathbf{g}}_{k}^{T} \mathbf{p}_{k}\right]\right) \\
= & \rho_{f} \eta_{k} \psi_{k} \\
\mathbb{E}\left[\left|A_{2, i}\right|^{2}\right] & =\mathbb{E}\left[\left(\sqrt{\rho_{f}} \hat{\mathbf{g}}_{k}^{T} \mathbf{p}_{i} \sqrt{\eta_{i}} s_{i}\right)^{H}\left(\sqrt{\rho_{f}} \hat{\mathbf{g}}_{k}^{T} \mathbf{p}_{i} \sqrt{\eta_{i}} s_{i}\right)\right] \\
& =\mathbb{E}\left[\sqrt{\rho_{f}} s_{i}^{*} \sqrt{\eta_{i}} \mathbf{p}_{i}^{H} \hat{\mathbf{g}}_{k}^{*} \sqrt{\rho_{f}} \hat{\mathbf{g}}_{k}^{T} \mathbf{p}_{i} \sqrt{\eta_{i}} s_{i}\right] \\
& =\rho_{f} \operatorname{tr}\left(\mathbb{E}\left[\sqrt{\eta_{i}} \mathbf{p}_{i}^{H} \hat{\mathbf{g}}_{k}^{*} \hat{\mathbf{g}}_{k}^{T} \mathbf{p}_{i} \sqrt{\eta_{i}} s_{i} s_{i}^{*}\right]\right) \\
& =\rho_{f} \operatorname{tr}\left(\eta_{i} \mathbb{E}\left[\mathbf{p}_{i}^{H} \hat{\mathbf{g}}_{k}^{*} \hat{\mathbf{g}}_{k}^{T} \mathbf{p}_{i}\right]\right) \\
& =\rho_{f} \eta_{i} \phi_{k, i}
\end{aligned}
$$

In the expressions above, we have

$$
\begin{gathered}
\psi_{k}=\mathbb{E}\left[\mathbf{p}_{k}^{H} \hat{\mathbf{g}}_{k}^{*} \hat{\mathbf{g}}_{k}^{T} \mathbf{p}_{k}\right], \text { for } k=1, \ldots, K, \\
\phi_{k, i}=\mathbb{E}\left[\mathbf{p}_{i}^{H} \hat{\mathbf{g}}_{k}^{*} \hat{\mathbf{g}}_{k}^{T} \mathbf{p}_{i}\right], \text { for } i \neq k, i=1, \ldots, K, k=1, \ldots, K, \\
\gamma_{k}=\operatorname{diag}\left\{\mathbb{E}\left[\mathbf{P}_{\mathrm{MMSE}}^{H} \mathbb{E}\left[\tilde{\mathbf{g}}_{k}^{*} \tilde{\mathbf{g}}_{k}^{T}\right] \mathbf{P}_{\mathrm{MMSE}}\right]\right\}, \text { for } k=1, \ldots, K,
\end{gathered}
$$

where $\mathbf{p}_{k}=\left[p_{1, k}, \ldots, p_{M, k}\right]^{T}$ is the column $k$ of matrix $\mathbf{P}_{\mathrm{MMSE}}, \psi_{k}$ is the $k$ th element of vector $\boldsymbol{\psi}, \phi_{k, i}$ is the $i$ th element of vector $\boldsymbol{\phi}_{k}, \gamma_{k, i}$ is the $i$ th element of vector $\boldsymbol{\gamma}_{k}$, and $\mathbb{E}\left[\tilde{\mathbf{g}}_{k}^{*} \tilde{\mathbf{g}}_{k}^{T}\right]$ is a diagonal matrix with $\left((1-n) \beta_{m, k}\right)$ on its $m$ th diagonal element.

By substituting (3-41), (3-42) and (3-43) in the SINR k,MMSE $_{\text {expression }}$ we get

$$
\mathrm{SINR}_{\mathrm{k}, \mathrm{MMSE}}=\frac{\rho_{f} \eta_{k} \psi_{k}}{\sigma_{w}^{2}+\rho_{f} \sum_{i=1, i \neq k}^{K} \eta_{i} \phi_{k, i}+\rho_{f} \sum_{i=1}^{K} \eta_{i} \gamma_{k, i}} .
$$

Note that in $\operatorname{SINR}_{\mathrm{k}, \mathrm{MMSE}}(\boldsymbol{\eta})$, the numerator and denominator are linear functions of $\boldsymbol{\eta}$. Consequently, $\operatorname{SINR}_{\mathrm{k}, \mathrm{MMSE}}(\boldsymbol{\eta})$ is a quasilinear function, 
enabling us to employ the bisection method [1].

In the optimization problems, when we express $\min _{k} \mathrm{SINR}_{k}$, we get the smallest element of the vector containing the SINR from all the $k$ th users, $k=1, \ldots, K$. In MATLAB, this is performed through the min function where $\min (\mathbf{u})$ represents the smallest value of vector $\mathbf{u}$.

\subsection{2}

\section{Computational Complexity}

Here, we evaluate the computational complexity of all techniques covered in this chapter [61].

Table 3.1: Computational Complexity

\begin{tabular}{|c|c|c|}
\hline \multirow{2}{*}{ APS Techniques } & LS-APS & $\mathcal{O}\left(M^{2}\right)$ \\
\cline { 2 - 3 } & ES-APS & $\mathcal{O}(L !)$ \\
\hline $\begin{array}{c}\text { Precoding } \\
+ \\
\text { Power Allocation }\end{array}$ & MMSE Precoder & $\mathcal{O}\left(M^{3}\right)$ \\
\cline { 2 - 3 } & ZF Precoder & $\mathcal{O}\left(M^{3}\right)$ \\
\cline { 2 - 3 } SINR Computation & ZF Precoder $[8,9]$ & $\mathcal{O}\left(M K^{2}\right)$ \\
\cline { 2 - 3 } & MPSE Precoder & $\mathcal{O}\left(M^{2} K^{2}\right)$ \\
\cline { 2 - 3 } & CB Precoder $[8,9]$ & $\mathcal{O}\left(M K^{2}\right)$ \\
\hline \multirow{3}{*}{ Power Allocation } & OPA & $\mathcal{O}\left(T_{\mathrm{OPA}} K^{3.5}\right)$ \\
\cline { 2 - 3 } & APA & $\mathcal{O}\left(T_{\mathrm{APA}} M K^{2}\right)$ \\
\cline { 2 - 3 } & UPA & $\mathcal{O}\left(M K^{2}\right)$ \\
\hline
\end{tabular}

In Table 3.1, we notice that the overall complexity of LS-APS with MMSE precoding and OPA is comparable to the same techniques applied to the existing ZF precoder from [9] and is higher than that of the CB precoder from $[8,9]$. Depending on the number of iterations of the bisection method, $T_{\mathrm{OPA}}$, the complexity of the OPA algorithm may prevail, or the one of the MMSE and ZF precoder, if $M^{3}>T_{\mathrm{OPA}} K^{3.5}$. The same can be said regarding the APA algorithm, depending on $M^{3}>T_{\mathrm{APA}} M K^{2}$, or the opposite. When evaluating the impact of APS on precoding and SINR computation, there is a reduction in computational complexity from $\mathcal{O}\left(M^{3}\right)$ to $\mathcal{O}\left((S N)^{3}\right)$ and from $\mathcal{O}\left(M^{2} K^{2}\right)$ to $\mathcal{O}\left((S N)^{2} K^{2}\right)$. For the power allocation techniques developed, APS only affects the APA and UPA algorithms, with a reduction in complexity 
from $\mathcal{O}\left(T_{\mathrm{APA}} M K^{2}\right)$ to $\mathcal{O}\left(T_{\mathrm{APA}}(S N) K^{2}\right)$ and from $\mathcal{O}\left(M K^{2}\right)$ to $\mathcal{O}\left((S N) K^{2}\right)$, respectively, where $M=L N>S N$. For the precoders with ES-APS, we notice that the complexity is $\mathcal{O}(L !)$, which is very costly when compared to LS-APS. Thus, for ES-APS we only investigate a very small system with $L=5$ singleantenna APs.

The complete calculations of computational cost are presented in Appendix A.

\section{5}

\section{Numerical Results}

In this section, we assess the proposed and existing techniques using simulations. The APS methods are previously applied, followed by the MMSE precoder with total power constraint, which is initially calculated with $\mathbf{N}=\mathbf{I}_{K}$, where $\mathbf{N} \in \mathbb{R}_{+}^{K \times K}$ is the power allocation diagonal matrix with $\sqrt{\eta_{1}}, \ldots, \sqrt{\eta_{K}}$ on its diagonal and $\eta_{k}$ is the power coefficient of user $k$. Next, power allocation is performed. With the corresponding power coefficients, the precoder is recomputed and the final power allocation is performed, completing two iterations in total.

We combined different precoding, power allocation and APS techniques. When describing an approach we will use the following notation:

- Precoding + Power Allocation + APS

For each category, we have the following methods:

- Precoding: CB, ZF, MMSE

- Power Allocation: OPA, APA and UPA

- APS: ES-APS and LS-APS

In the following simulations, the parameter $n$ is used to define the amount of CSI imperfection, being the adjustable percentage between $\alpha_{m, k}$ and $\beta_{m, k}$, $\alpha_{m, k}=n \beta_{m, k}$, where $0 \leq n \leq 1$. Remember that $\alpha_{m, k}$ is the variance of $\hat{g}_{m, k}$ and $\beta_{m, k}$ is the LS coefficient. If we consider, for example, $10 \%$ of CSI imperfection, $n=0.9$. Furthermore, we declare the average transmitted power as $E_{t r}$, the total number of antenna elements as $M$ and the maximum transmitted power of each antenna as $\rho_{f}$. In all experiments, we performed 120 channel realizations and assumed $\sigma_{s}^{2}=1$, where $\sigma_{s}^{2}$ is the symbol variance.

A comparison between the iterative MMSE precoder with OPA and UPA with the $\mathrm{CB}$ and $\mathrm{ZF}$ precoders from [8,9] is presented in Fig. 3.2, in terms of sum-rate vs. SNR. In the first case, both ES-APS and LS-APS are compared to a non selective scheme (NS). Considering the high complexity of ES-APS, a 
very small system is analysed in Fig. 3.2 and Fig. 3.3 with $L=5$ APs, $N=1$ antenna element each, $S=3$ selected APs and $K=2$ single-antenna users only. In Fig. 3.3, we explore the same scenario, but instead of looking at OPA, we examine APA.

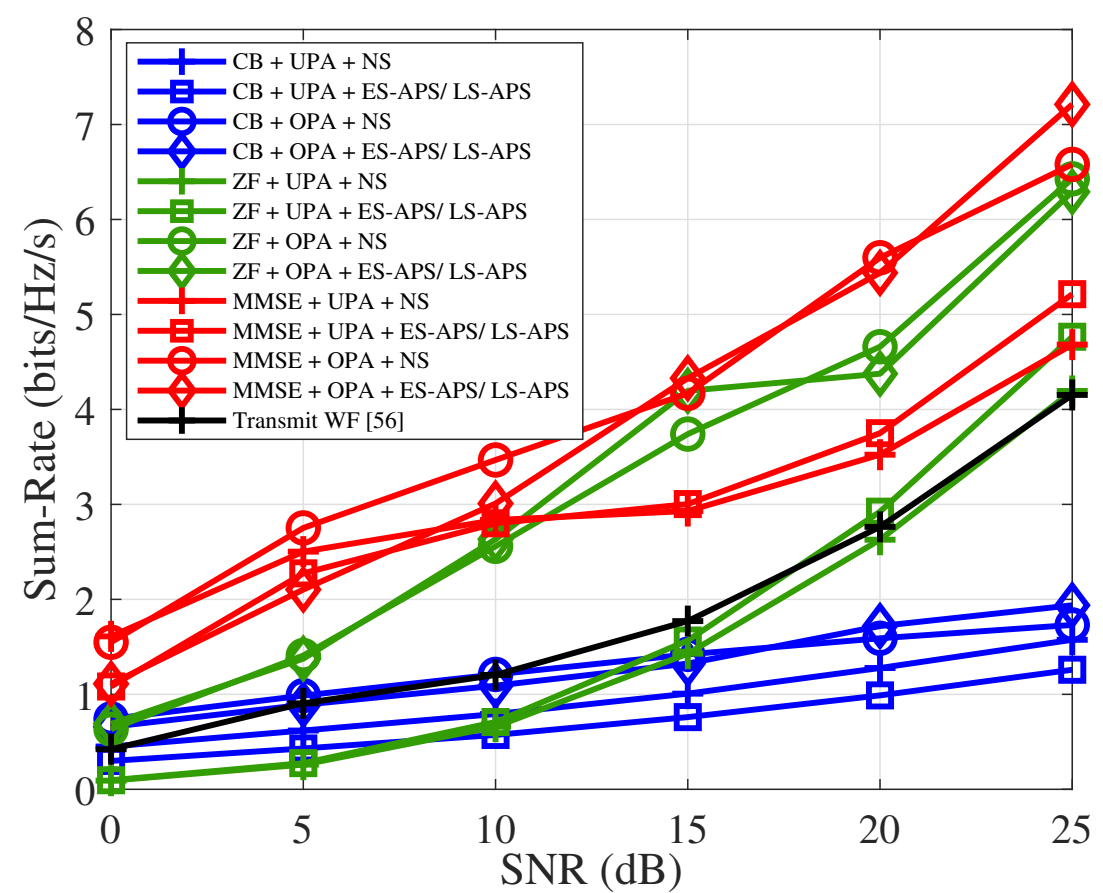

Figure 3.2: Sum-Rate vs. SNR with $L=5, \mathrm{~N}=1, S=3, K=2, n=0.99,120$ channel realizations and $E_{t r}=M \rho_{f}$.

As shown in Fig. 3.2, MMSE + OPA is the approach with the best performance. Furthermore, it is visible that MMSE + UPA achieves higher rates than $\mathrm{ZF}+\mathrm{OPA}$ for lower SNR values and it is better than ZF + UPA in the whole experiment. Additionally, we can see that the application of ESAPS/LS-APS generates comparable or even improved results for MMSE, ZF and $\mathrm{CB}+\mathrm{OPA}$. In the case of $\mathrm{CB}+\mathrm{UPA}$, there is a small degradation in performance when applying APS techniques. We remark that both selection schemes are comparable and are illustrated together for aesthetic purposes. For this reason we can conclude that the suboptimal method is an effective replacement for the optimal solution, with the advantage of having lower computational complexity.

Fig. 3.3 provides us some insight on the performance of the MMSE + APA when compared to MMSE + UPA, and ZF. The uniform and adaptive technique have a better performance than ZF + OPA for lower values of SNR. Moreover, we can see that the MMSE+APA performs better than MMSE+UPA. In all combined techniques, OPA performs better than UPA and 
APA. In both figures, the transmit WF from [56] shows a degraded performance due to its lack of appropriate power allocation.

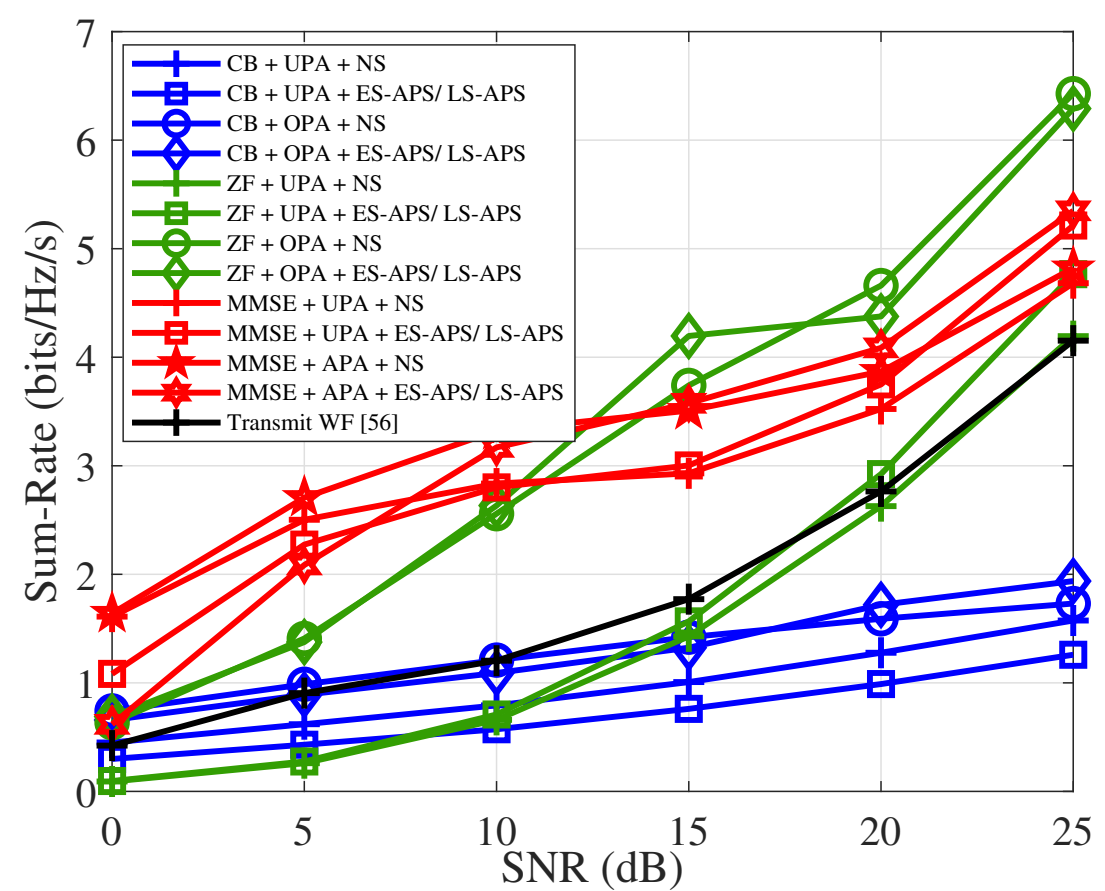

Figure 3.3: Sum-Rate vs. SNR with $L=5, \mathrm{~N}=1, S=3, K=2, n=0.99,120$ channel realizations and $E_{t r}=M \rho_{f}$.

In the second experiment, we explore LS-APS in a large system with $L=128$ APs, $N=1$ antenna element each, $S=64$ selected APs and $K=16$ single-antenna users, in terms of minimum SINR and sum-rate. As we did previously, in Fig. 3.4 and Fig. 3.6 we compare the $\mathrm{CB}$ and $\mathrm{ZF}$ precoders from $[8,9]$ with OPA and UPA and in Fig. 3.5 and Fig. 3.7 we substitute OPA for APA.

The results in Fig. 3.4 and Fig. 3.5 validate the functionality of the maxmin fairness power allocation algorithm, where our main objective was that the OPA algorithms have at least the same minimum SINR than UPA ones, if not higher. In higher SINR values, we can see the considerable improvement provided by the OPA algorithm, when compared to UPA. When it comes to the APA solution, it is comparable to the MMSE+UPA in terms of minimum SINR. 
Chapter 3. Iterative AP Selection, MMSE Precoding and Power Allocation for Cell-Free Massive MIMO Systems

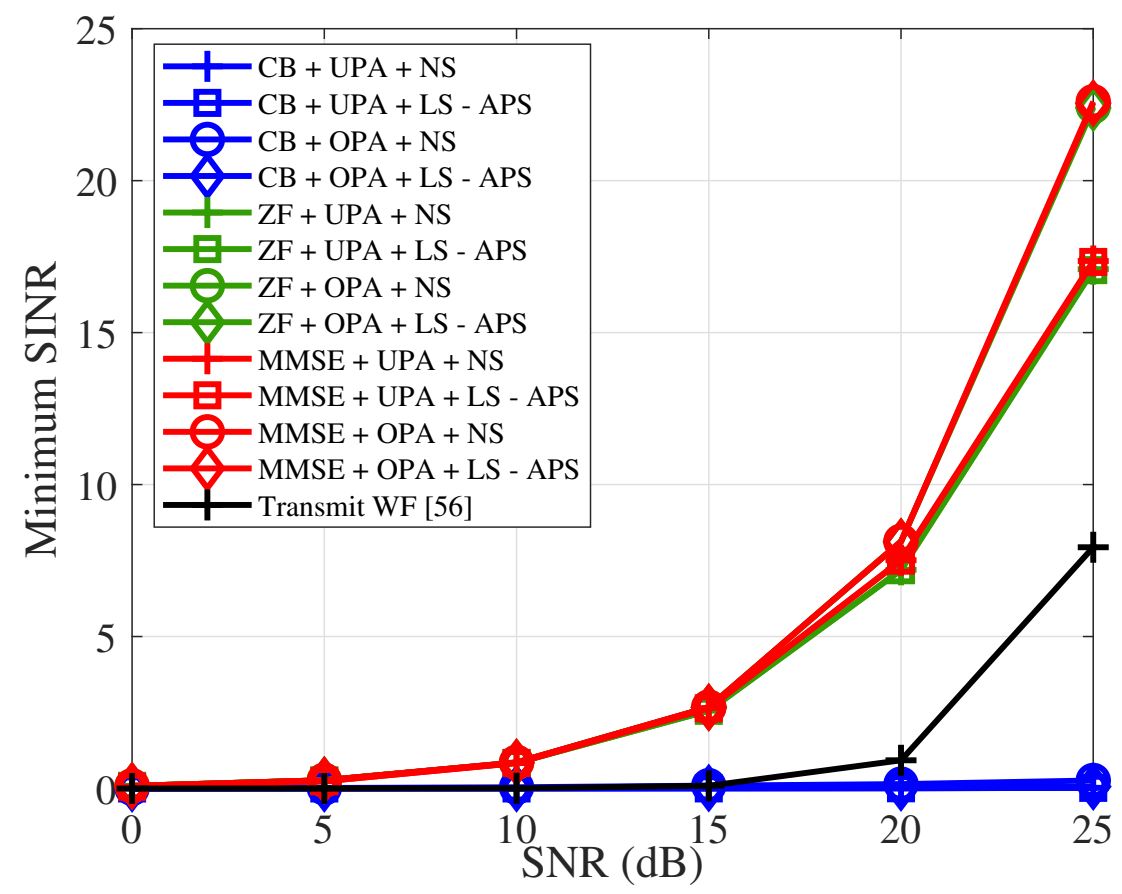

Figure 3.4: Minimum SINR vs. SNR with $L=128, \mathrm{~N}=1, S=64, K=16$, $n=0.99,120$ channel realizations and $E_{t r}=M \rho_{f}$.

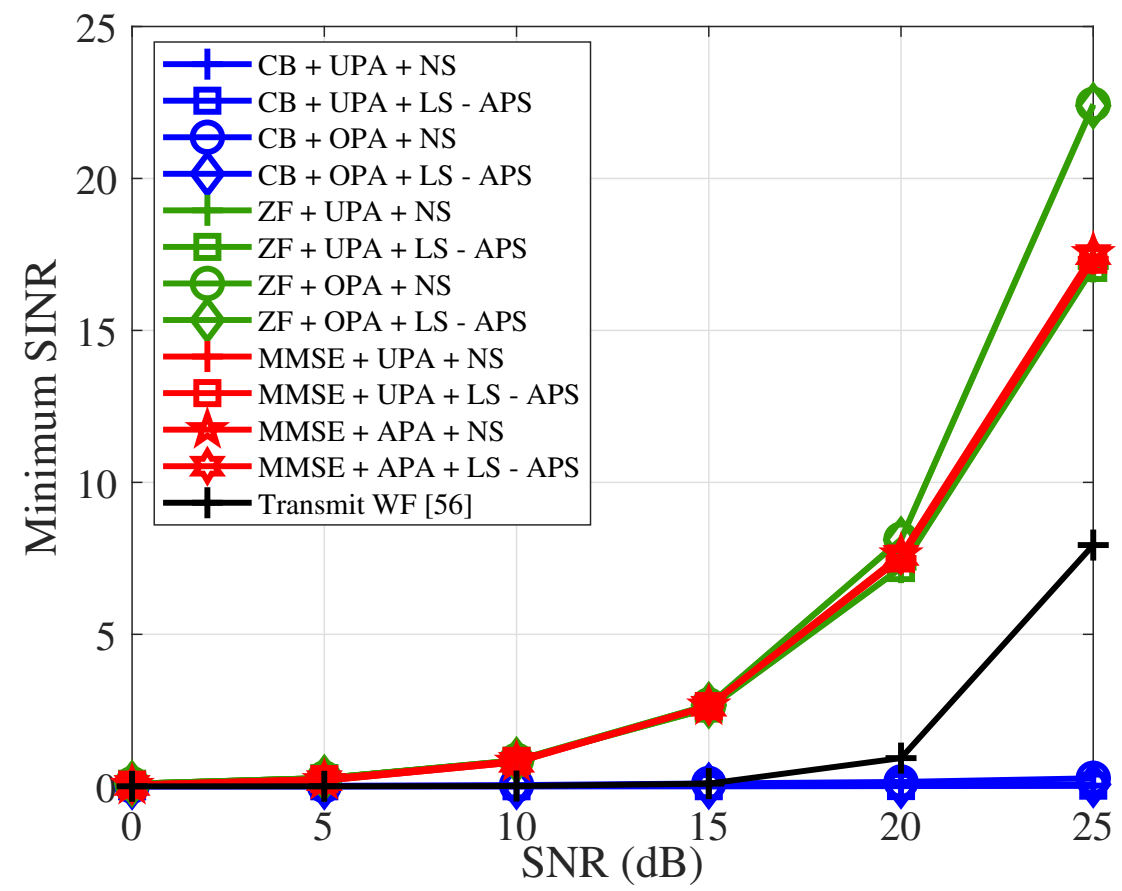

Figure 3.5: Minimum SINR vs. SNR with $L=128, \mathrm{~N}=1, S=64, K=16$, $n=0.99,120$ channel realizations and $E_{t r}=M \rho_{f}$.

In Fig. 3.6, the MMSE + OPA has the best performance compared to the other schemes. Moreover, the MMSE + UPA achieves higher rates than ZF + 
UPA. In addition, we note that performance is maintained when applying LSAPS for large systems, except for CB + OPA, when performance is improved. For all precoders, OPA provides significantly better rates than UPA. In a larger system, the performance of the transmit filter from [56] is not as good as MMSE+UPA due to its inappropriate design for cell-free systems.

We notice in Fig. 3.7 that MMSE + APA can provide an improvement in performance when compared to MMSE + UPA, in terms of sum-rate. Therefore, it is an attractive solution in comparison with the remaining precoders combined with UPA.

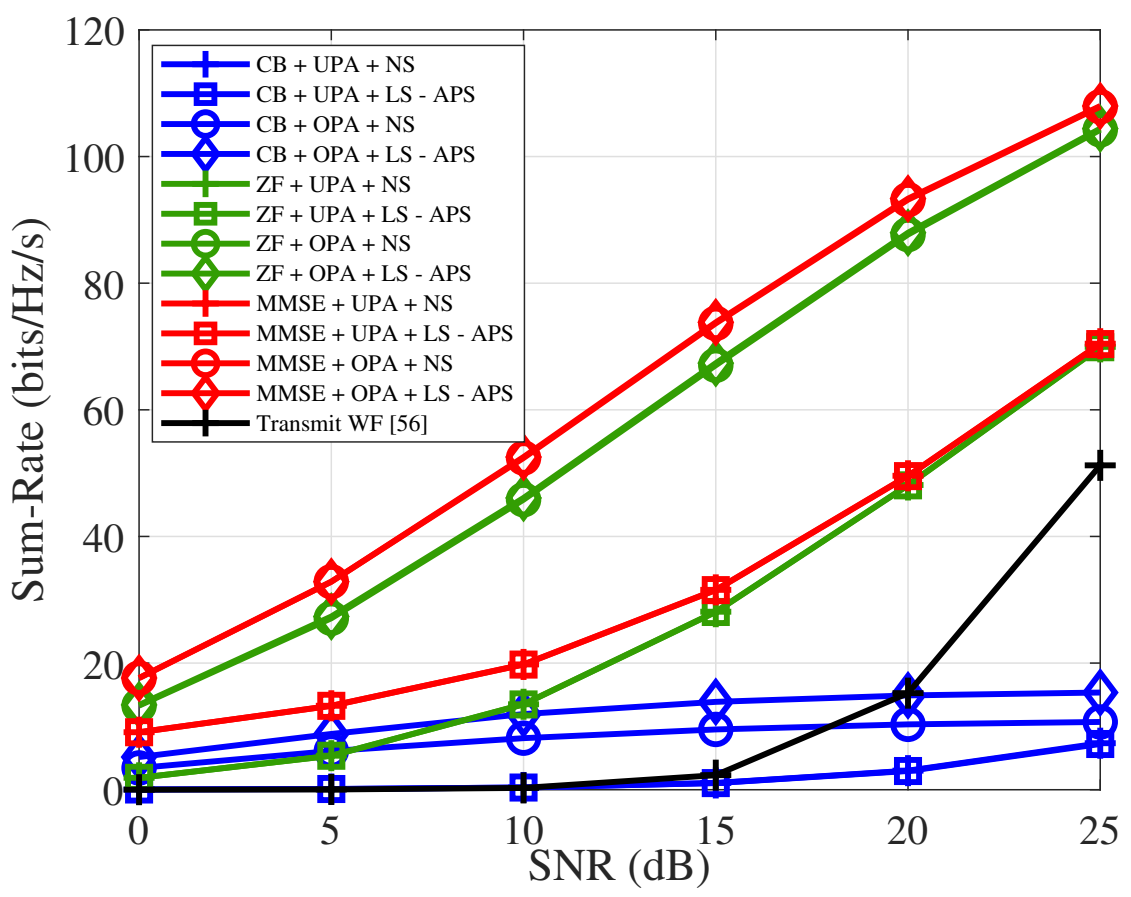

Figure 3.6: Sum-Rate vs. SNR with $L=128, \mathrm{~N}=1, S=64, K=16, n=0.99$, 120 channel realizations and $E_{t r}=M \rho_{f}$.

It is important to emphasize that improving the minimum SINR or the sum-rate is a matter of adjusting the parameters of the bisection method (Algorithm 1). By increasing $t_{b}$ we force the minimum SINR to go higher but the tendency is that the sum-rate decreases. When the problem is relaxed, meaning, when $t_{b}$ is decreased, the sum-rate gets improved but the minimum SINR is lower. This is a common characteristic of max-min problems and the big question is whether to decide if the requirement is that all users get at least an specific rate or if the system as a whole needs to have a certain performance. 


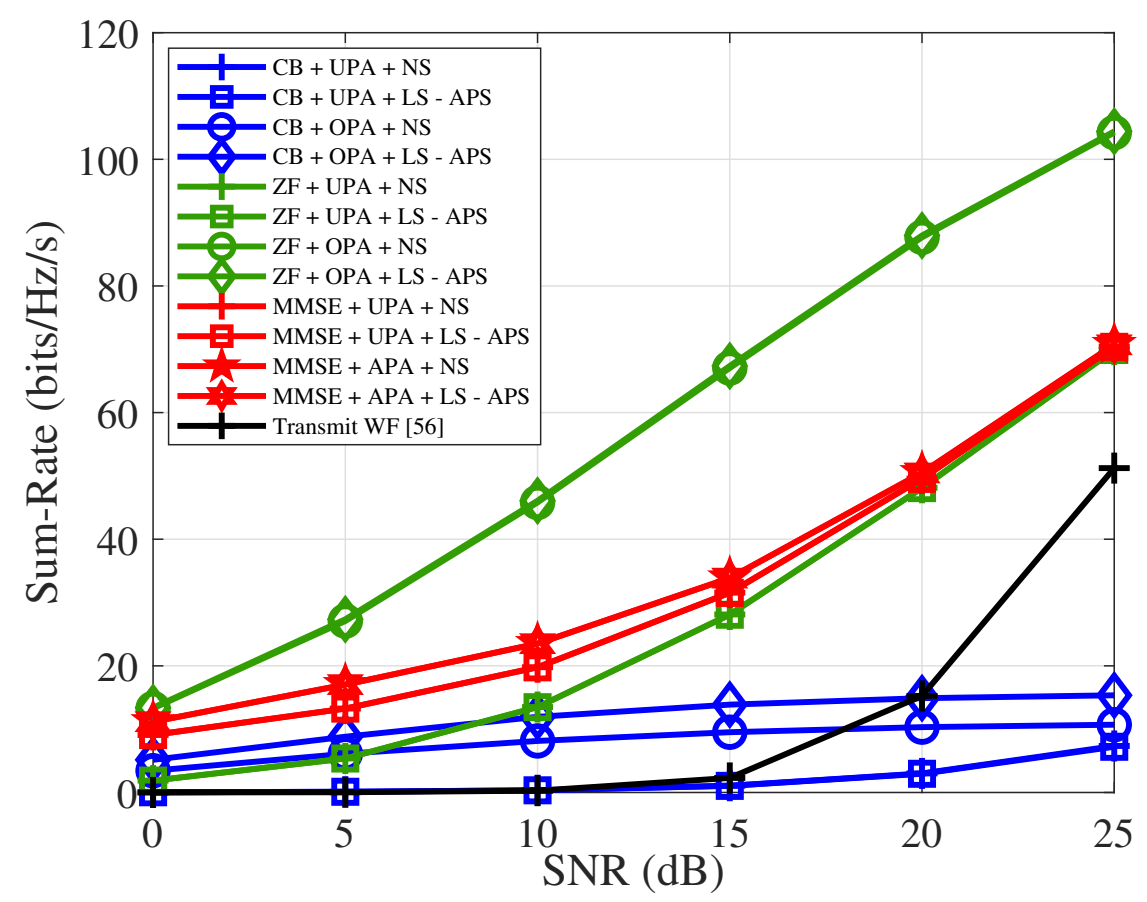

Figure 3.7: Sum-Rate vs. SNR with $L=128, \mathrm{~N}=1, S=64, K=16, n=0.99$, 120 channel realizations and $E_{t r}=M \rho_{f}$.

Similarly, the next experiment considers a large system, now in terms of BER vs. SNR using multiple-antenna APs. We assume perfect CSI $(n=1)$, Quadrature Phase Shift Keying (QPSK) modulation and 100 symbols per packet. We test LS-APS with an antenna array of $N=4$ elements each, $L=24$ APs (total of $M=96$ antennas), $S=12$ selected APs (total of 48 selected antennas) and $K=8$ users.

As in experiment 2, LS-APS causes no degradation in performance, with the benefit of reducing the number of active APs by half. The insight provided by Fig. 3.8 and Fig. 3.9 is the same as before. MMSE + OPA has the best performance when compared to other precoders and OPA performs better when applied to all precoding designs. We also emphasize here that MMSE + APA is a promising approach against UPA and improves performance. Besides, we remark that in terms of BER, for higher values of SNR, MMSE + APA is comparable to $\mathrm{ZF}+\mathrm{OPA}$.

The final experiment gives an insight on how the number of selected APs in LS-APS affects the system performance in terms of BER vs. SNR. We consider $L=24$ with $N=4$ elements each (total of $M=96$ antennas) and $K=8$ users. We vary the number of $S$ selected APs considering $L, L / 2, L / 3, L / 4, L / 6$ and $L / 8$. 


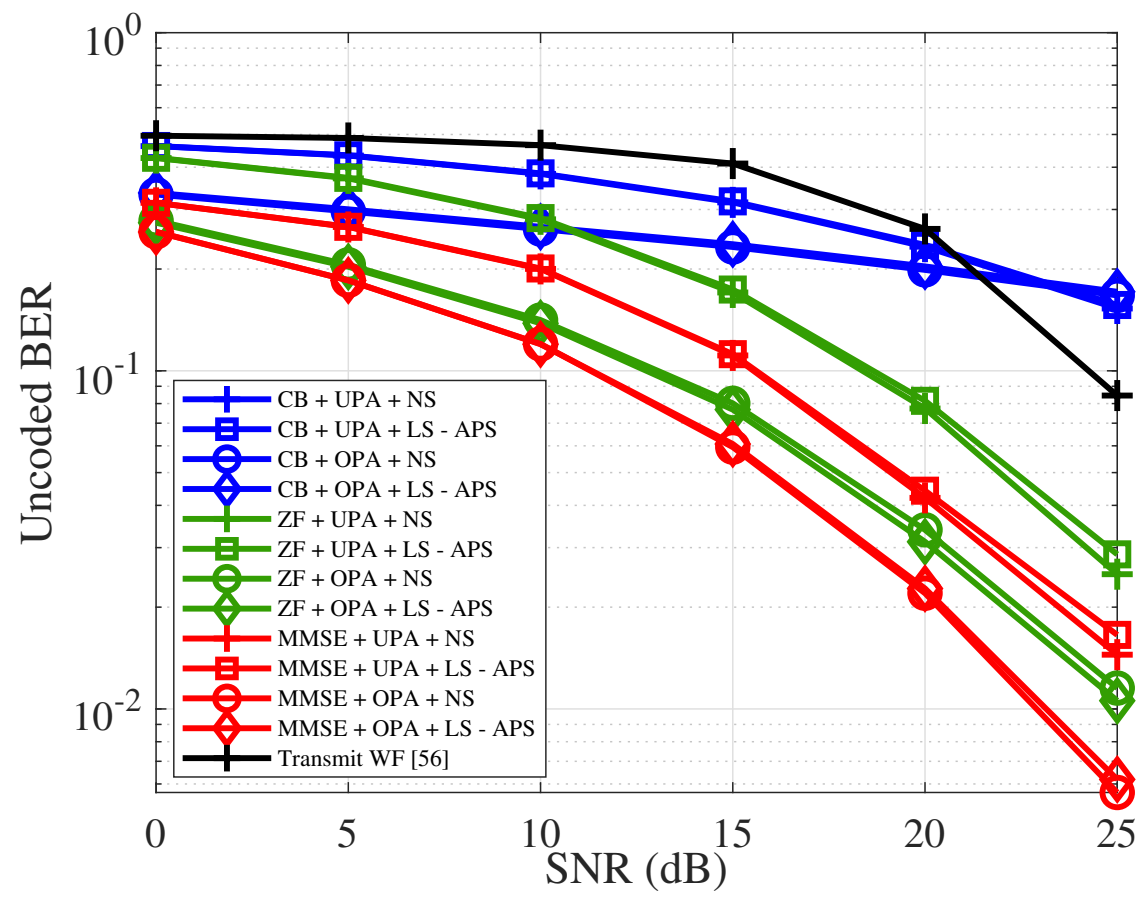

Figure 3.8: BER vs. SNR with $L=24, N=4, S=12, K=8, n=1,120$ channel realizations, 100 symbols per packet and $E_{t r}=M \rho_{f}$.

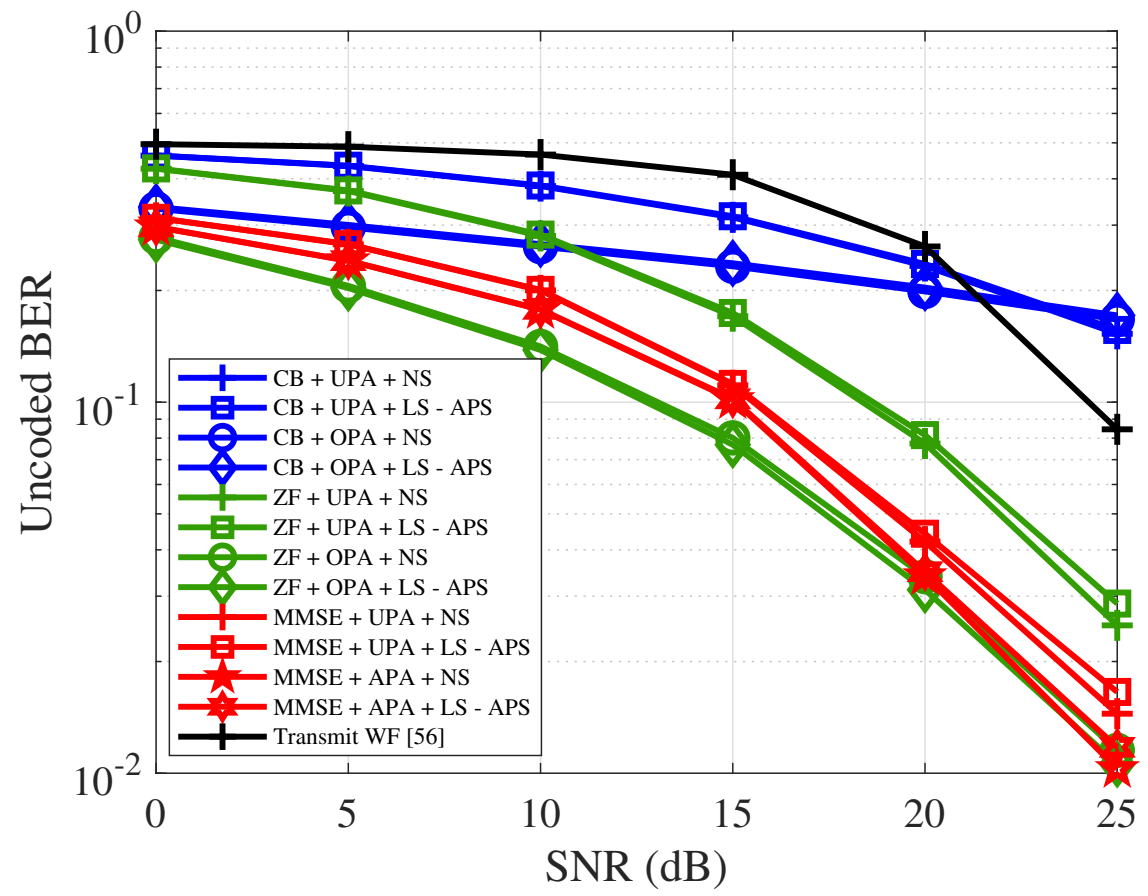

Figure 3.9: BER vs. SNR with $L=24, N=4, S=12, K=8, n=1,120$ channel realizations, 100 symbols per packet and $E_{t r}=M \rho_{f}$.

As we can see in Fig. 3.10, when we compare $S=24$ with $S=3$ curves, a difference of $4.6 \mathrm{~dB}$ can be seen in $\mathrm{BER}=4 \times 10^{-2}$. In the same $\mathrm{BER}$ value, the difference between $S=24$ and $S=4$ is of $2 \mathrm{~dB}$. For BER $=2 \times 10^{-2}$, 
the difference between $S=24$ and $S=8$ is of $1.45 \mathrm{~dB}$. We can conclude that by decreasing the number of selected APs in $66 \%$, the performance does not suffer much degradation.

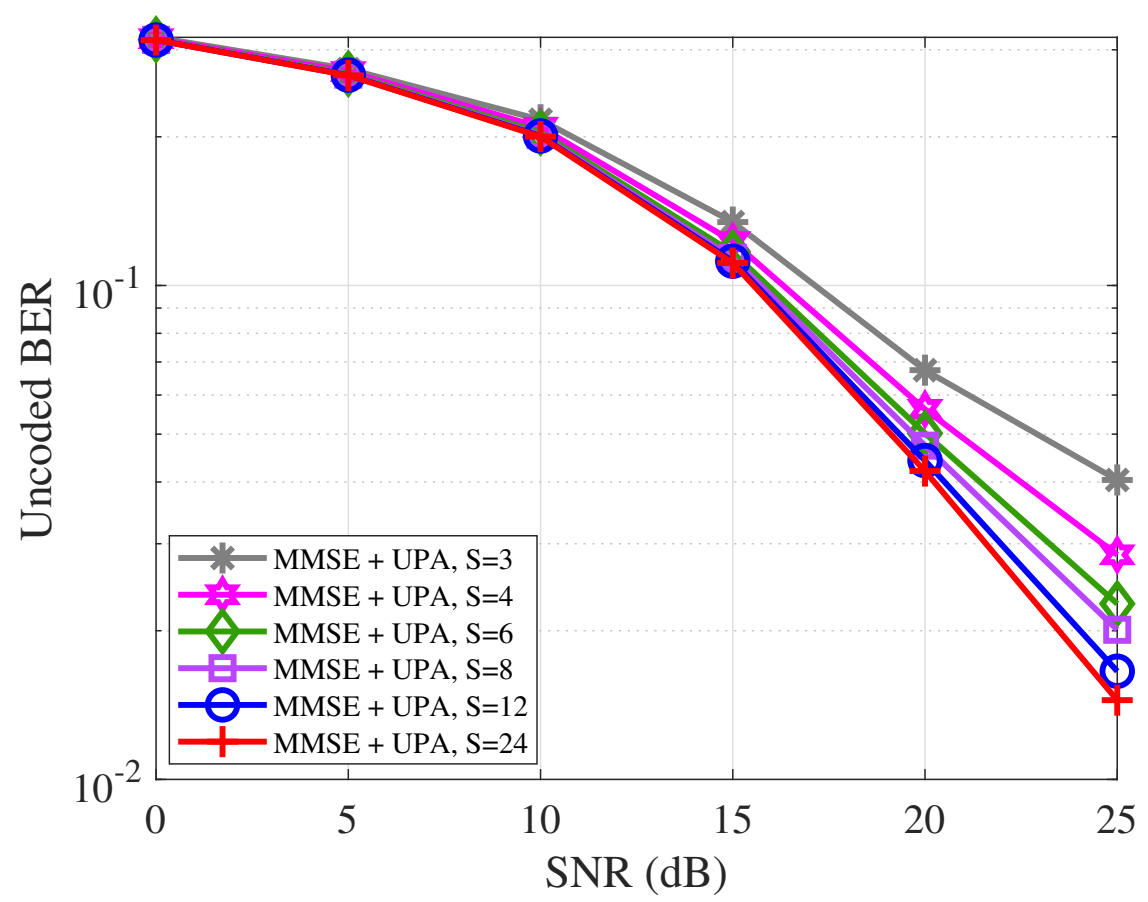

Figure 3.10: BER vs. SNR with $L=24, N=4, K=8, n=1,120$ channel realizations, 100 symbols per packet and $E_{t r}=M \rho_{f}$.

\section{6 \\ Summary}

We have introduced iterative APS, MMSE precoding and power allocation techniques for the downlink of a Cell-Free Massive MIMO system with single and multiple-antenna APs, in the presence of perfect and imperfect CSI. Two APS schemes were studied, one based on ES, which takes the experiment to its optimal scenario and the other, less complex but with comparable performance, based on the largest LS coefficients. An MMSE precoder has been developed by taking into account a power allocation matrix in its derivation. Subsequently, three power control algorithms are elaborated, OPA, APA and UPA, with different performances, criteria and computational complexities. We have derived sum-rate expressions along with a study of the computational cost of all techniques. Simulations show that the designed techniques outperform existing approaches and can reduce computational complexity. 


\section{4 \\ Iterative Robust MMSE Precoding and Power Allocation for Cell-Free Massive MIMO Systems}

In this chapter, we introduce an iterative linear robust MMSE (RMMSE) precoding scheme based on the total power constraint. In a similar way to the approach developed in Section 3.2, a power allocation matrix is taken into account in its derivation. Then, optimal and uniform power allocation schemes are introduced, based on the max-min fairness approach and the robust design. A sum-rate analysis is carried out based on the proposed robust scheme, and the computational complexity of the projected techniques is evaluated. In the end, numerical results demonstrating the potential of the methods are displayed.

In Section 4.1 we derive the RMMSE precoder with total power constraint. In Section 4.2, OPA and UPA schemes adapted to the robust approach are explained. A sum-rate analysis and the computational complexity of the designs are developed in Section 4.3. In Section 4.4, numerical results are presented and conclusions are drawn in Section 4.5.

\section{1}

\section{Iterative RMMSE Precoder with Total Power Constraint}

As an extension of the precoder from Section 3.2, a robust version is now derived, in the interest of mitigating the effects of channel estimation errors. Here, we also consider the power allocation matrix $\mathbf{N}$ in the derivation and use the CSI matrix $\hat{\mathbf{G}}$ and the CSI error matrix $\tilde{\mathbf{G}}$ to derive the method. An iterative linear RMMSE precoder with power allocation is considered as well, as a means to maximize the minimum SINR. In the derivation we insert a factor $f^{-1}$ at the receiver which can be interpreted as an automatic gain control [56]. All the variables related to this precoder have the subscript "RMMSE".

As in (3-6), the received signal by the $k$ th user is given by

$$
y_{k}=\sqrt{\rho_{f}} \mathbf{g}_{k}^{T} \mathbf{P} \mathbf{N ~ s}+w_{k} .
$$

For all the users combined, the received signal is 


$$
\begin{aligned}
& \mathbf{y}=\sqrt{\rho_{f}} \mathbf{G}^{T} \mathbf{P} \mathbf{N s}+\mathbf{w} \\
& \mathbf{y}=\sqrt{\rho_{f}}(\hat{\mathbf{G}}+\tilde{\mathbf{G}})^{T} \mathbf{P} \mathbf{N s}+\mathbf{w} \\
& \mathbf{y}=\sqrt{\rho_{f}} \hat{\mathbf{G}}^{T} \mathbf{P} \mathbf{N s}+\underbrace{\sqrt{\rho_{f}} \tilde{\mathbf{G}}^{T} \mathbf{P} \mathbf{N s}}_{\boldsymbol{\Delta}}+\mathbf{w} .
\end{aligned}
$$

The design of the proposed RMMSE precoder has two main objectives: to minimize the MSE under a total power constraint and to mitigate the effects of the channel estimation error. The optimal solution would be $\mathbb{E}\left[\|\boldsymbol{\Delta}\|_{2}^{2}\right] \rightarrow 0$, as in a system with perfect CSI. Therefore, we will insert it as a constraint, with the aid of a auxiliary variable $\theta$, in order to minimize the effects of this second term. The constant $\rho_{f}$ will be taken out of this proposed constraint since it does not affect the solution.

To obtain the RMMSE precoder, we solve the following optimization:

$$
\begin{aligned}
& \left\{\mathbf{P}_{\text {RMMSE }}, \mathbf{N}, f_{\text {RMMSE }}\right\}=\operatorname{argmin}_{\{\mathbf{P}, \mathbf{N}, f\}} \mathbb{E}\left[\left\|\mathbf{s}-f^{-1} \mathbf{y}\right\|_{2}^{2}\right] \\
& \text { s.t.: } \theta \mathbb{E}\left[\left\|\tilde{\mathbf{G}}^{T} \mathbf{P} \mathbf{N} \mathbf{s}\right\|_{2}^{2}\right]=0 \\
& \text { and } \mathbb{E}\left[\|\mathbf{x}\|_{2}^{2}\right]=E_{t r}
\end{aligned}
$$

where the average transmitted power is described by

$$
\mathbb{E}\left[\|\mathbf{x}\|_{2}^{2}\right]=\rho_{f} \operatorname{tr}\left(\mathbf{P N C} \mathbf{C}_{\mathbf{s}} \mathbf{N}^{H} \mathbf{P}^{H}\right)=E_{t r}
$$

The proposed constraint can be rewritten as

$$
\begin{aligned}
\theta \mathbb{E}\left[\left\|\tilde{\mathbf{G}}^{T} \mathbf{P} \mathbf{N} \mathbf{s}\right\|_{2}^{2}\right] & =\theta \mathbb{E}\left[\left(\tilde{\mathbf{G}}^{T} \mathbf{P} \mathbf{N} \mathbf{s}\right)^{H}\left(\tilde{\mathbf{G}}^{T} \mathbf{P} \mathbf{N} \mathbf{s}\right)\right] \\
& =\theta \mathbb{E}\left[\mathbf{s}^{H} \mathbf{N}^{H} \mathbf{P}^{H} \mathbb{E}\left[\tilde{\mathbf{G}}^{*} \tilde{\mathbf{G}}^{T}\right] \mathbf{P N \mathbf { s }}\right] \\
& =\theta \operatorname{tr}\left(\mathbb{E}\left[\mathbf{s}^{H} \mathbf{N}^{H} \mathbf{P}^{H} \mathbb{E}\left[\tilde{\mathbf{G}}^{*} \tilde{\mathbf{G}}^{T}\right] \mathbf{P N \mathbf { s }}\right]\right) \\
& =\theta \mathbb{E}\left[\operatorname{tr}\left(\mathbf{N}^{H} \mathbf{P}^{H} \mathbb{E}\left[\tilde{\mathbf{G}}^{*} \tilde{\mathbf{G}}^{T}\right] \mathbf{P} \mathbf{N} \mathbf{s s}^{H}\right)\right] \\
& =\theta \operatorname{tr}\left(\mathbb{E}\left[\tilde{\mathbf{G}}^{*} \tilde{\mathbf{G}}^{T}\right] \mathbf{P} \mathbf{N} \mathbf{C}_{\mathbf{s}} \mathbf{N}^{H} \mathbf{P}^{H}\right) \\
& =\operatorname{tr}\left(\mathbf{M P N C} \mathbf{N}_{\mathbf{s}} \mathbf{N}^{H} \mathbf{P}^{H}\right) .
\end{aligned}
$$

The auxiliary matrix $\mathbf{M}$ is defined as

$$
\mathbf{M}=\theta \mathbb{E}\left[\tilde{\mathbf{G}}^{*} \tilde{\mathbf{G}}^{T}\right]
$$

where $\mathbb{E}\left[\tilde{\mathbf{G}}^{*} \tilde{\mathbf{G}}^{T}\right]$ is a diagonal matrix with $\sum_{k=1}^{K}(1-n) \beta_{m, k}$ on its $m$ th diagonal element and $\theta$ is a chosen scalar. Although $\mathbf{M}$ is diagonal, it does not fit in the diagonal loading technique, since the diagonal elements are not equal, $\mathbf{M} \neq u \mathbf{I}_{M}$. The proposed constraint is categorized as a generalized loading, as studied in [45], where the matrix is obtained through steering vector errors 
and complemented by a scalar. Similar to this technique, we propose a matrix $\mathbf{M}$ obtained from the statistics of the channel estimation error matrix $\tilde{\mathbf{G}}$ and scaled by a constant $\theta$.

By constructing the Lagrangian function with the Lagrange multipliers, $\lambda$ and $\Gamma$, setting its derivatives to zero and considering a power allocation matrix $\mathbf{N}$, we can compute the precoder $\mathbf{P}$ and the normalization factor $f$, as

$$
\begin{aligned}
\mathcal{L}(\mathbf{P}, \mathbf{N}, f, \Gamma, \lambda)=\mathbb{E} & {\left[\left\|\mathbf{s}-f^{-1} \mathbf{y}\right\|_{2}^{2}\right]+\Gamma\left(\operatorname{tr}\left(\mathbf{M} \mathbf{P} \mathbf{N C}_{s} \mathbf{N}^{H} \mathbf{P}^{H}\right)-0\right) } \\
& +\lambda\left(\rho_{f} \operatorname{tr}\left(\mathbf{P} \mathbf{N C}_{s} \mathbf{N}^{H} \mathbf{P}^{H}\right)-E_{t r}\right) \\
=\operatorname{tr} & \left(\mathbf{C}_{s}\right)-f^{-1} \sqrt{\rho_{f}} \operatorname{tr}\left(\hat{\mathbf{G}}^{T} \mathbf{P} \mathbf{N C}_{s}\right)-f^{-1} \sqrt{\rho_{f}} \operatorname{tr}\left(\hat{\mathbf{G}}^{*} \mathbf{C}_{s} \mathbf{N}^{H} \mathbf{P}^{H}\right) \\
& +f^{-2} \rho_{f} \operatorname{tr}\left(\hat{\mathbf{G}}^{*} \hat{\mathbf{G}}^{T} \mathbf{P} \mathbf{N C}_{s} \mathbf{N}^{H} \mathbf{P}^{H}\right)+f^{-2} \operatorname{tr}\left(\mathbf{C}_{w}\right) \\
& +\Gamma \operatorname{tr}\left(\mathbf{M} \mathbf{P} \mathbf{N C}_{s} \mathbf{N}^{H} \mathbf{P}^{H}\right)+\lambda\left(\rho_{f} \operatorname{tr}\left(\mathbf{P N C} \mathbf{N}_{s} \mathbf{N}^{H}\right)-E_{t r}\right) .
\end{aligned}
$$

Using the result of the partial derivative, $\partial \operatorname{tr}\left(\mathbf{B X}^{H}\right) / \partial \mathbf{X}^{*}=\mathbf{B}$, we obtain the following expressions:

$$
\begin{aligned}
\frac{\partial \mathcal{L}(\mathbf{P}, \mathbf{N}, f, \Gamma, \lambda)}{\partial \mathbf{P}^{*}}=- & f^{-1} \sqrt{\rho_{f}} \hat{\mathbf{G}}^{*} \mathbf{C}_{s} \mathbf{N}^{H}+f^{-2} \rho_{f} \hat{\mathbf{G}}^{*} \hat{\mathbf{G}}^{T} \mathbf{P} \mathbf{N} \mathbf{C}_{s} \mathbf{N}^{H}+\Gamma \mathbf{M} \mathbf{P} \mathbf{N C}_{s} \mathbf{N}^{H} \\
& +\lambda \rho_{f} \mathbf{P} \mathbf{N C}_{s} \mathbf{N}^{H}=0
\end{aligned}
$$

and

$$
\begin{aligned}
\frac{\partial \mathcal{L}(\mathbf{P}, \mathbf{N}, f, \Gamma, \lambda)}{\partial f}=f^{-2} & \sqrt{\rho_{f}} \operatorname{tr}\left(\hat{\mathbf{G}}^{T} \mathbf{P} \mathbf{N C}_{s}\right)+f^{-2} \sqrt{\rho_{f}} \operatorname{tr}\left(\hat{\mathbf{G}}^{*} \mathbf{C}_{s} \mathbf{N}^{H} \mathbf{P}^{H}\right) \\
& -2 f^{-3} \rho_{f} \operatorname{tr}\left(\hat{\mathbf{G}}^{*} \hat{\mathbf{G}}^{T} \mathbf{P} \mathbf{N C}_{s} \mathbf{N}^{H} \mathbf{P}^{H}\right)-2 f^{-3} \operatorname{tr}\left(\mathbf{C}_{w}\right)=0
\end{aligned}
$$

Solving for (4-8), we get:

$$
\begin{aligned}
& -f^{-1} \sqrt{\rho_{f}} \hat{\mathbf{G}}^{*} \mathbf{C}_{s} \mathbf{N}^{H}+f^{-2} \rho_{f} \hat{\mathbf{G}}^{*} \hat{\mathbf{G}}^{T} \mathbf{P} \mathbf{N C}_{s} \mathbf{N}^{H}+\Gamma \mathbf{M} \mathbf{P} \mathbf{N C}_{s} \mathbf{N}^{H}+\lambda \rho_{f} \mathbf{P} \mathbf{N C}_{s} \mathbf{N}^{H}=0 \\
& f^{-1} \sqrt{\rho_{f}} \hat{\mathbf{G}}^{*} \mathbf{C}_{s} \mathbf{N}^{H}=f^{-2} \rho_{f} \hat{\mathbf{G}}^{*} \hat{\mathbf{G}}^{T} \mathbf{P} \mathbf{N C}_{s} \mathbf{N}^{H}+\Gamma \mathbf{M} \mathbf{P} \mathbf{C}_{s} \mathbf{N}^{H}+\lambda \rho_{f} \mathbf{P} \mathbf{N C}_{s} \mathbf{N}^{H} \\
& f \sqrt{\rho_{f}} \hat{\mathbf{G}}^{*} \mathbf{C}_{s} \mathbf{N}^{H}=\rho_{f} \hat{\mathbf{G}}^{*} \hat{\mathbf{G}}^{T} \mathbf{P} \mathbf{N C}_{s} \mathbf{N}^{H}+\Gamma f^{2} \mathbf{M} \mathbf{P} \mathbf{N C}_{s} \mathbf{N}^{H}+\lambda f^{2} \rho_{f} \mathbf{P} \mathbf{N C}_{s} \mathbf{N}^{H} \\
& f \sqrt{\rho_{f}} \hat{\mathbf{G}}^{*}=\rho_{f} \hat{\mathbf{G}}^{*} \hat{\mathbf{G}}^{T} \mathbf{P} \mathbf{N}+\Gamma f^{2} \mathbf{M} \mathbf{P} \mathbf{N}+\lambda f^{2} \rho_{f} \mathbf{P N} \\
& f \sqrt{\rho_{f}} \hat{\mathbf{G}}^{*}=\rho_{f}\left(\hat{\mathbf{G}}^{*} \hat{\mathbf{G}}^{T}+\frac{\Gamma f^{2}}{\rho_{f}} \mathbf{M}+\lambda f^{2} \mathbf{I}_{M}\right) \mathbf{P N}
\end{aligned}
$$


Chapter 4. Iterative Robust MMSE Precoding and Power Allocation for

$$
\begin{aligned}
& \mathbf{P N}=\frac{f}{\sqrt{\rho_{f}}} \underbrace{\left(\hat{\mathbf{G}}^{*} \hat{\mathbf{G}}^{T}+\frac{\Gamma f^{2}}{\rho_{f}} \mathbf{M}+\lambda f^{2} \mathbf{I}_{M}\right)^{-1} \hat{\mathbf{G}}^{*}}_{\tilde{\mathbf{P}}} \\
& \mathbf{P}=\frac{f}{\sqrt{\rho_{f}}} \underbrace{\left(\hat{\mathbf{G}}^{*} \hat{\mathbf{G}}^{T}+\frac{\Gamma f^{2}}{\rho_{f}} \mathbf{M}+\lambda f^{2} \mathbf{I}_{M}\right)^{-1} \hat{\mathbf{G}}^{*} \mathbf{N}^{-1}=\frac{f}{\sqrt{\rho_{f}}} \tilde{\mathbf{P}} \mathbf{N}^{-1} .}_{\tilde{\mathbf{P}}}
\end{aligned}
$$

By using the expression in (4-9), we arrive at

$$
\begin{aligned}
& f^{-2} \sqrt{\rho_{f}} \operatorname{tr}\left(\hat{\mathbf{G}}^{T} \mathbf{P} \mathbf{N} \mathbf{C}_{s}\right)+f^{-2} \sqrt{\rho_{f}} \operatorname{tr}\left(\hat{\mathbf{G}}^{*} \mathbf{C}_{s} \mathbf{N}^{H} \mathbf{P}^{H}\right)-2 f^{-3} \rho_{f} \operatorname{tr}\left(\hat{\mathbf{G}}^{*} \hat{\mathbf{G}}^{T} \mathbf{P} \mathbf{N} \mathbf{C}_{s} \mathbf{N}^{H} \mathbf{P}^{H}\right) \\
& -2 f^{-3} \operatorname{tr}\left(\mathbf{C}_{w}\right)=0 \\
& 2 f^{-2} \sqrt{\rho_{f}} \operatorname{Re}\left(\operatorname{tr}\left(\hat{\mathbf{G}}^{*} \mathbf{C}_{s} \mathbf{N}^{H} \mathbf{P}^{H}\right)\right)=2 f^{-3} \rho_{f} \operatorname{tr}\left(\hat{\mathbf{G}}^{*} \hat{\mathbf{G}}^{T} \mathbf{P} \mathbf{N} \mathbf{C}_{s} \mathbf{N}^{H} \mathbf{P}^{H}\right)+2 f^{-3} \operatorname{tr}\left(\mathbf{C}_{w}\right) \\
& f \sqrt{\rho_{f}} \operatorname{tr}\left(\hat{\mathbf{G}}^{*} \mathbf{C}_{s} \mathbf{N}^{H} \mathbf{P}^{H}\right)=\rho_{f} \operatorname{tr}\left(\hat{\mathbf{G}}^{*} \hat{\mathbf{G}}^{T} \mathbf{P} \mathbf{N C}_{s} \mathbf{N}^{H} \mathbf{P}^{H}\right)+\operatorname{tr}\left(\mathbf{C}_{w}\right) .
\end{aligned}
$$

Multiplying from the right $\mathbf{C}_{s} \mathbf{N}^{H} \mathbf{P}^{H}$ in (4-10), using the trace operator and considering $\epsilon=\lambda f^{2}$, the expression takes the following form:

$$
\begin{aligned}
f \sqrt{\rho_{f}} \operatorname{tr}\left(\hat{\mathbf{G}}^{*} \mathbf{C}_{s} \mathbf{N}^{H} \mathbf{P}^{H}\right)=\rho_{f} \operatorname{tr}\left(\hat{\mathbf{G}}^{*} \hat{\mathbf{G}}^{T} \mathbf{P} \mathbf{N C}_{s} \mathbf{N}^{H} \mathbf{P}^{H}\right)+\Gamma f^{2} \operatorname{tr}\left(\mathbf{M P N C} \mathbf{N}_{s} \mathbf{N}^{H} \mathbf{P}^{H}\right) \\
+\epsilon \rho_{f} \operatorname{tr}\left(\mathbf{P} \mathbf{N C}_{s} \mathbf{N}^{H} \mathbf{P}^{H}\right) .
\end{aligned}
$$

Equalling expression (4-11) to (4-12), we have

$$
\begin{aligned}
& \rho_{f} \operatorname{tr}\left(\hat{\mathbf{G}}^{*} \hat{\mathbf{G}}^{T} \mathbf{P} \mathbf{N} \mathbf{C}_{s} \mathbf{N}^{H} \mathbf{P}^{H}\right)+\operatorname{tr}\left(\mathbf{C}_{w}\right)=\rho_{f} \operatorname{tr}\left(\hat{\mathbf{G}}^{*} \hat{\mathbf{G}}^{T} \mathbf{P} \mathbf{N C}_{s} \mathbf{N}^{H} \mathbf{P}^{H}\right) \\
& +\Gamma f^{2} \operatorname{tr}\left(\mathbf{M P N C} \mathbf{N}_{s} \mathbf{N}^{H} \mathbf{P}^{H}\right)+\epsilon \rho_{f} \operatorname{tr}\left(\mathbf{P} \mathbf{N C}_{s} \mathbf{N}^{H} \mathbf{P}^{H}\right) \\
& \operatorname{tr}\left(\mathbf{C}_{w}\right)=\Gamma f^{2} \operatorname{tr}\left(\mathbf{M} \mathbf{P} \mathbf{N C}_{s} \mathbf{N}^{H} \mathbf{P}^{H}\right)+\epsilon \rho_{f} \operatorname{tr}\left(\mathbf{P} \mathbf{N C}_{s} \mathbf{N}^{H} \mathbf{P}^{H}\right)
\end{aligned}
$$

Since

$$
\begin{aligned}
E_{t r} & =\rho_{f} \operatorname{tr}\left(\mathbf{P} \mathbf{N C}_{s} \mathbf{N}^{H} \mathbf{P}^{H}\right) \\
& =\rho_{f} \operatorname{tr}\left(\frac{f}{\sqrt{\rho_{f}}} \tilde{\mathbf{P}} \mathbf{N}^{-1} \mathbf{N C}_{s} \mathbf{N}^{H}\left(\mathbf{N}^{-1}\right)^{H} \tilde{\mathbf{P}}^{H} \frac{f}{\sqrt{\rho_{f}}}\right) \\
& =f^{2} \operatorname{tr}\left(\tilde{\mathbf{P}} \mathbf{C}_{s} \tilde{\mathbf{P}}^{H}\right),
\end{aligned}
$$

it yields 


$$
\begin{aligned}
& \operatorname{tr}\left(\mathbf{C}_{w}\right)=\Gamma f^{2} \operatorname{tr}\left(\mathbf{M P N} \mathbf{N}_{s} \mathbf{N}^{H} \mathbf{P}^{H}\right)+\epsilon E_{t r} \\
& \epsilon=\frac{\operatorname{tr}\left(\mathbf{C}_{w}\right)}{E_{t r}}-\frac{\Gamma f^{2} \operatorname{tr}\left(\mathbf{M P N C} \mathbf{N}_{s} \mathbf{N}^{H} \mathbf{P}^{H}\right)}{E_{t r}} \\
& \epsilon=\frac{\operatorname{tr}\left(\mathbf{C}_{w}\right)}{E_{t r}}-\frac{\Gamma f^{2}}{E_{t r}} \operatorname{tr}\left(\mathbf{M} \frac{f}{\sqrt{\rho_{f}}} \tilde{\mathbf{P}} \mathbf{N}^{-1} \mathbf{N} \mathbf{C}_{s} \mathbf{N}^{H} \frac{f}{\sqrt{\rho_{f}}}\left(\mathbf{N}^{-1}\right)^{H} \tilde{\mathbf{P}}^{H}\right) \\
& \epsilon=\frac{\operatorname{tr}\left(\mathbf{C}_{w}\right)}{E_{t r}}-\frac{\Gamma f^{4} \operatorname{tr}\left(\mathbf{M} \tilde{\mathbf{P}} \mathbf{C}_{s} \tilde{\mathbf{P}}^{H}\right)}{\rho_{f} E_{t r}},
\end{aligned}
$$

and

$$
f=f_{\mathrm{RMMSE}}=\sqrt{\frac{E_{t r}}{\operatorname{tr}\left(\tilde{\mathbf{P}} \mathbf{C}_{s} \tilde{\mathbf{P}}^{H}\right)}} .
$$

Therefore, the RMMSE precoder is

$$
\begin{aligned}
\mathbf{P}_{\mathrm{RMMSE}}= & \frac{f_{\mathrm{RMMSE}}}{\sqrt{\rho_{f}}}\left(\hat{\mathbf{G}}^{*} \hat{\mathbf{G}}^{T}+\frac{\operatorname{tr}\left(\mathbf{C}_{w}\right)}{E_{t r}} \mathbf{I}_{M}\right. \\
& \left.+\frac{\Gamma f_{\mathrm{RMMSE}}^{2}\left(E_{t r} \mathbf{M}-f_{\mathrm{RMMSE}}^{2} \operatorname{tr}\left(\mathbf{M} \tilde{\mathbf{P}} \mathbf{C}_{s} \tilde{\mathbf{P}}^{H}\right) \mathbf{I}_{M}\right)}{\rho_{f} E_{t r}}\right)^{-1} \hat{\mathbf{G}}^{*} \mathbf{N}^{-1} \\
= & \frac{f_{\mathrm{RMMSE}}}{\sqrt{\rho_{f}}} \tilde{\mathbf{P}} \mathbf{N}^{-1},
\end{aligned}
$$

where $\operatorname{tr}\left(\mathbf{C}_{w}\right)=K \sigma_{w}^{2}$.

Note that if we assume perfect CSI, $\tilde{\mathbf{G}}=\mathbf{0}_{M \times K}$, or set $\Gamma=0$ then the RMMSE precoder becomes the MMSE precoder for cell-free. Thus, the advantages of the RMMSE precoder will be only perceived in an imperfect CSI scenario.

For the RMMSE precoder to outperform the one in Section 3.2, it is necessary that the diagonal elements of $\mathbf{H}$ are nonnegative, where

$$
\mathbf{H}=\frac{\Gamma f_{\mathrm{RMMSE}}^{2}\left(E_{t r} \mathbf{M}-f_{\mathrm{RMMSE}}^{2} \operatorname{tr}\left(\mathbf{M} \tilde{\mathbf{P}} \mathbf{C}_{s} \tilde{\mathbf{P}}^{H}\right) \mathbf{I}_{M}\right)}{\rho_{f} E_{t r}} .
$$

Similarly to the technique presented in Chapter 3, we perform power allocation after computing the robust precoder. With a new matrix $\mathbf{N}$, we recompute $\mathbf{P}_{\text {RMMSE }}$. Finally, we repeat the computation of $\mathbf{N}$ to find $\mathbf{N}_{\text {RMMSE }}$, by using $\mathbf{P}_{\text {RMMSE }}$ in the calculation. It is important to highlight that the power allocation matrix $\mathbf{N}$ present in $\mathbf{P}_{\text {RMMSE }}$ is different from the final $\mathbf{N}_{\text {RMMSE }}$. Thus, they will not cancel each other.

In Algorithm 6 we show how to compute the RMMSE precoder with power allocation. The technique requires pre calculated values for $\tilde{\mathbf{P}}[1]$ and $f_{\text {RMMSE }}[1]$. Therefore, first, we assume these values to be the same from the MMSE precoder from Chapter 3. Then, to calculate the RMMSE precoder 
we have to set values of $\Gamma$ and $\theta$. Throughout the experiments, we noticed that the coefficients of $\mathbf{H}$ had to be greater or equal to 0 , but could not have higher values than $10^{3} \frac{\operatorname{tr}\left(\mathbf{C}_{w}\right)}{E_{t r}} \mathbf{I}_{M}$, as to avoid performance deterioration. It was necessary to first turn the elements into positive, by setting $\theta=-1$, and then regulate the matrix around $10^{2} \frac{\operatorname{tr}\left(\mathbf{C}_{w}\right)}{E_{t r}} \mathbf{I}_{M}$, with the application of $\Gamma$, so as to maximize the SINR of the system. After regulating $\Gamma[i+1]$, we obtain our new $\tilde{\mathbf{P}}[i+1]$ and $f_{\text {RMMSE }}[i+1]$. Fixing these values, we enter the power allocation loop, as done in the iterative MMSE precoder from the previous chapter. We start with $\mathbf{N}[1]$ equal to the final $\mathbf{N}_{\text {MMSE }}$ and then iteratively find its own $\mathbf{N}_{\text {RMMSE }}$. Each of the loops perform two iterations in total, meaning that $\operatorname{ITER}_{\text {prec }}=\operatorname{ITER}_{\mathrm{pa}}=2$.

Algorithm 6 Iterative RMMSE Precoding With Total Power Constraint (RMMSE)

1: Initialize $f_{\mathrm{RMMSE}}[1]=f_{\mathrm{MMSE}}, \theta=-1, \mathbf{N}[1]=\mathbf{N}_{\mathrm{MMSE}}$, ITER prec $_{\text {(number }}$

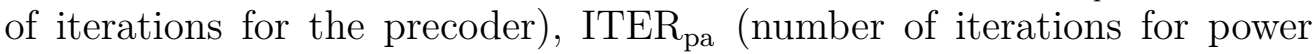
allocation).

2: Initialize $\tilde{\mathbf{P}}[1]=\left(\hat{\mathbf{G}}^{*} \hat{\mathbf{G}}^{T}+\frac{\operatorname{tr}\left(\mathbf{C}_{w}\right)}{E_{t r}} \mathbf{I}_{M}\right)^{-1} \hat{\mathbf{G}}^{*}$

3: For $\mathrm{i}=1: \mathrm{ITER}_{\text {prec }}$

Update $\Gamma, \tilde{\mathbf{P}}$ and $f_{\text {RMMSE }}$ :

4: Calculate $\Gamma[i+1]$ to optimize the SINR

5: Calculate $\tilde{\mathbf{P}}[i+1] \leftarrow(4-17)$

6: Calculate $f_{\mathrm{RMMSE}}[i+1]=\sqrt{\frac{E_{t r}}{\operatorname{tr}\left(\tilde{\mathbf{P}}[i+1] \mathbf{C}_{s} \tilde{\mathbf{P}}[i+1]^{H}\right)}}$

7: end for

8: Obtain $\tilde{\mathbf{P}}=\tilde{\mathbf{P}}[i+1]$ and $f_{\mathrm{RMMSE}}=f_{\mathrm{RMMSE}}[i+1]$

9: For $\mathrm{j}=1: \mathrm{ITER}_{\mathrm{pa}}$

10: Calculate $\mathbf{P}_{\text {RMMSE }}[j] \leftarrow(4-17)$

11: Calculate $\mathbf{N}[j+1] \leftarrow(4-22)$ or $(4-23)$ (with fixed $\left.\mathbf{P}_{\text {RMMSE }}[j]\right)$

12: end for

13: Obtain $\mathbf{P}_{\text {RMMSE }}=\mathbf{P}_{\text {RMMSE }}[j]$ and $\mathbf{N}_{\text {RMMSE }}=\mathbf{N}[j+1]$.

\section{2}

\section{Robust Power Allocation}

In this section we introduce OPA and UPA techniques, now applied to the RMMSE precoder. The objective is to find the power allocation matrix $\mathbf{N}$, which will be used to recompute the robust precoder $\mathbf{P}_{\mathrm{RMMSE}}$ and the final $\mathbf{N}_{\text {RMMSE }}$.

\subsection{1}

\section{Optimal Power Allocation (OPA)}

Similarly to the optimization problem from Section 3.3.1, the maxmin fairness power allocation problem with antenna power constraint for the 
RMMSE precoder can be formulated as

$$
\begin{aligned}
& \max _{\boldsymbol{\eta}} \min _{k} \operatorname{SINR}_{k}(\boldsymbol{\eta}) \\
& \text { s.t. } \sum_{i=1}^{K} \eta_{i} \delta_{m, i} \leq 1, m=1, \ldots, M,
\end{aligned}
$$

where

$$
\mathrm{SINR}_{k}=\frac{\mathbb{E}\left[\left|A_{1}\right|^{2}\right]}{\sigma_{w}^{2}+\sum_{i=1, i \neq k}^{K} \mathbb{E}\left[\left|A_{2, i}\right|^{2}\right]+\mathbb{E}\left[\left|A_{3}\right|^{2}\right]}
$$

is the SINR of user $k, A_{1}$ is the desired signal, $\sigma_{w}^{2}$ is the noise variance, $A_{2, i}$ is the interference caused by user $i$ in user $k$, for $i \neq k, i=1, \ldots, K$ and $A_{3}$ is the CSI error.

We also define

$$
\boldsymbol{\delta}_{m}=\operatorname{diag}\left\{\mathbb{E}\left[\mathbf{p}_{m}^{T} \mathbf{p}_{m}^{*}\right]\right\}, m=1, \ldots, M,
$$

where $\mathbf{p}_{m}=\left[p_{m, 1}, \ldots, p_{m, K}\right]$ is the $m$ th row of the precoder $\mathbf{P}_{\mathrm{RMMSE}}$ and $\delta_{m, i}$ is the $i$ th element of vector $\boldsymbol{\delta}_{m}$.

The power allocation problem expressed in an epigraph form to employ the bisection method is

$$
\begin{aligned}
& \text { find } \boldsymbol{\eta} \\
& \text { s.t. } \operatorname{SINR}_{\mathrm{k}}(\boldsymbol{\eta}) \geq t, k=1, \ldots, K, \\
& \qquad \sum_{i=1}^{K} \eta_{i} \delta_{m, i} \leq 1, m=1, \ldots, M,
\end{aligned}
$$

where $t=\frac{t_{b}+t_{e}}{2}$ is the midpoint of a chosen interval $\left(t_{b}, t_{e}\right)$ that contains the optimal value $t^{*}$, as in [1].

\subsection{2}

\section{Uniform Power Allocation (UPA)}

As in Section 3.3.3, we also present an alternative to the OPA scheme, based on [9]. Imagining a situation where a certain antenna element $m$ transmits with full power and all $\eta_{k}$, for $k=1, \ldots, K$ are equal and at their minimum possible value, we have

$$
\eta_{k}=1 /\left(\max _{m} \sum_{i=1}^{K} \delta_{m, i}\right), k=1, \ldots, K,
$$

where $\delta_{m, i}$ is the $i$ th element of vector $\boldsymbol{\delta}_{m}$. 


\section{3}

\section{Analysis}

In this section, a sum-rate analysis is derived and the computational complexity of the investigated scheme is evaluated.

\subsection{1}

\section{Sum-Rate}

Expanding expression (4-1), we obtain:

$$
\begin{aligned}
& y_{k}=\sqrt{\rho_{f}} \mathbf{g}_{k}^{T} \mathbf{P}_{\text {RMMSE }} \mathbf{N}_{\text {RMMSE }} \mathbf{s}+w_{k} \\
& =\sqrt{\rho_{f}}\left(\hat{\mathbf{g}}_{k}+\tilde{\mathbf{g}}_{k}\right)^{T} \mathbf{P}_{\text {RMMSE }} \mathbf{N}_{\text {RMMSE }} \mathbf{s}+w_{k} \\
& =\underbrace{\sqrt{\rho_{f}} \hat{\mathbf{g}}_{k}^{T} \mathbf{P}_{\mathrm{RMMSE}} \mathbf{N}_{\mathrm{RMMSE}} \mathbf{S}}_{\text {desired signal }+ \text { interference }}+\underbrace{\sqrt{\rho_{f}} \tilde{\mathbf{g}}_{k}^{T} \mathbf{P}_{\mathrm{RMMSE}} \mathbf{N}_{\mathrm{RMMSE}} \mathbf{S}}_{\text {CSI error }}+w_{k},
\end{aligned}
$$

Assuming Gaussian signalling, the achievable rate of the $k$ th user with the iterative RMMSE precoder is equal to

$$
R_{k, \text { RMMSE }}=\log _{2}\left(1+\operatorname{SINR}_{k, \mathrm{RMMSE}}\right) .
$$

The sum-rate is expressed by

$$
R_{\mathrm{RMMSE}}=\sum_{k=1}^{K} \log _{2}\left(1+\operatorname{SINR}_{k, \mathrm{RMMSE}}\right),
$$

where

$$
\begin{gathered}
\operatorname{SINR}_{k, \text { RMMSE }}=\frac{\mathbb{E}\left[\left|A_{1}\right|^{2}\right]}{\sigma_{w}^{2}+\sum_{i=1, i \neq k}^{K} \mathbb{E}\left[\left|A_{2, i}\right|^{2}\right]+\mathbb{E}\left[\left|A_{3}\right|^{2}\right]}, \\
A_{1}=\sqrt{\rho_{f}} \hat{\mathbf{g}}_{k}^{T} \mathbf{p}_{k} \sqrt{\eta_{k}} s_{k},
\end{gathered}
$$

is the desired signal by the $k$ th user,

$$
A_{2, i}=\sqrt{\rho_{f}} \hat{\mathbf{g}}_{k}^{T} \mathbf{p}_{i} \sqrt{\eta_{i}} s_{i}, \text { for } i \neq k, i=1, \ldots, K,
$$

is the interference caused by user $i$ in $k$ and

$$
A_{3}=\sqrt{\rho_{f}} \tilde{\mathbf{g}}_{k}^{T} \mathbf{P}_{\mathrm{RMMSE}} \mathbf{N}_{\mathrm{RMMSE}} \mathbf{S}
$$

is the CSI error.

As calculated before, the mean-square values of $A_{1}, A_{2, i}$ and $A_{3}$ are:

$$
\begin{gathered}
\mathbb{E}\left[\left|A_{1}\right|^{2}\right]=\rho_{f} \eta_{k} \psi_{k}, \text { for } k=1, \ldots, K, \\
\mathbb{E}\left[\left|A_{2, i}\right|^{2}\right]=\rho_{f} \eta_{i} \phi_{k, i}, \text { for } i \neq k, i=1, \ldots, K, k=1, \ldots, K, \\
\mathbb{E}\left[\left|A_{3}\right|^{2}\right]=\rho_{f} \sum_{i=1}^{K} \eta_{i} \gamma_{k, i}, \text { for } i=1, \ldots, K, k=1, \ldots, K
\end{gathered}
$$

where 


$$
\begin{gathered}
\psi_{k}=\mathbb{E}\left[\mathbf{p}_{k}^{H} \hat{\mathbf{g}}_{k}^{*} \hat{\mathbf{g}}_{k}^{T} \mathbf{p}_{k}\right], \text { for } k=1, \ldots, K, \\
\phi_{k, i}=\mathbb{E}\left[\mathbf{p}_{i}^{H} \hat{\mathbf{g}}_{k}^{*} \hat{\mathbf{g}}_{k}^{T} \mathbf{p}_{i}\right], \text { for } i \neq k, i=1, \ldots, K, k=1, \ldots, K, \\
\gamma_{k}=\operatorname{diag}\left\{\mathbb{E}\left[\mathbf{P}_{\mathrm{RMMSE}}^{H} \mathbb{E}\left[\tilde{\mathbf{g}}_{k}^{*} \tilde{\mathbf{g}}_{k}^{T}\right] \mathbf{P}_{\mathrm{RMMSE}}\right]\right\}, \text { for } k=1, \ldots, K,
\end{gathered}
$$

$\mathbf{p}_{k}=\left[p_{1, k}, \ldots, p_{M, k}\right]^{T}$ is the column $k$ of matrix $\mathbf{P}_{\mathrm{RMMSE}}, \psi_{k}$ is the $k$ th element of vector $\boldsymbol{\psi}, \phi_{k, i}$ is the $i$ th element of vector $\boldsymbol{\phi}_{k}, \gamma_{k, i}$ is the $i$ th element of vector $\gamma_{k}$, and $\mathbb{E}\left[\tilde{\mathbf{g}}_{k}^{*} \tilde{\mathbf{g}}_{k}^{T}\right]$ is a diagonal matrix with $\left((1-n) \beta_{m, k}\right)$ on its $m$ th diagonal element.

By substituting (4-31), (4-32) and (4-33) in the SINR k,RMMSE $_{\text {Rpression }}$ we get

$$
\operatorname{SINR}_{\mathrm{k}, \mathrm{RMMSE}}=\frac{\rho_{f} \eta_{k} \psi_{k}}{\sigma_{w}^{2}+\rho_{f} \sum_{i=1, i \neq k}^{K} \eta_{i} \phi_{k, i}+\rho_{f} \sum_{i=1}^{K} \eta_{i} \gamma_{k, i}} .
$$

In the expression above, the numerator and denominator are linear functions of $\boldsymbol{\eta}$, creating a quasilinear function, meaning that the bisection method can be used [1].

\subsection{2}

\section{Computational Complexity with RMMSE}

Now we will update Table 3.1 with the investigated methods of this chapter.

As shown in Table 4.1, the complexity of the RMMSE precoder is comparable to the MMSE and the ZF precoder from [9]. The CB precoder, $[8,9]$, however, presents much lower computational complexity, at the cost of degraded performance. The same can be said in terms of SINR computation. If $M^{3}>T_{\text {OPA }} K^{3.5}$, the computational cost of RMMSE + OPA will be $\mathcal{O}\left(M^{3}\right)$. Depending on the number of iterations of the bisection method, $T_{\mathrm{OPA}}$, the OPA scheme may prevail when applied to all precoders. In contrast, if UPA is applied, it will not affect the complexity of the RMMSE, MMSE and ZF techniques. We conclude that the proposed RMMSE precoder has comparable computational cost to previous methods but can outperform existing precoders in the presence of imperfect CSI, as shown in Section 4.4.

The results presented in this table were obtained in Appendix A. 
Table 4.1: Computational Complexity with RMMSE

\begin{tabular}{|c|c|c|}
\hline \multirow{2}{*}{ APS Techniques } & LS-APS & $\mathcal{O}\left(M^{2}\right)$ \\
\hline & ES-APS & $\mathcal{O}(L !)$ \\
\hline \multirow{4}{*}{$\begin{array}{c}\text { Precoding } \\
+ \\
\text { Power Allocation }\end{array}$} & MMSE Precoder & $\mathcal{O}\left(M^{3}\right)$ \\
\hline & RMMSE Precoder & $\mathcal{O}\left(M^{3}\right)$ \\
\hline & ZF Precoder & $\mathcal{O}\left(M^{3}\right)$ \\
\hline & CB Precoder $[8,9]$ & $\mathcal{O}(M K)$ \\
\hline \multirow{4}{*}{ SINR Computation } & MMSE Precoder & $\mathcal{O}\left(M^{2} K^{2}\right)$ \\
\hline & RMMSE Precoder & $\mathcal{O}\left(M^{2} K^{2}\right)$ \\
\hline & ZF Precoder & $\mathcal{O}\left(M^{2} K^{2}\right)$ \\
\hline & CB Precoder $[8,9]$ & $\mathcal{O}\left(M K^{2}\right)$ \\
\hline \multirow{3}{*}{ Power Allocation } & OPA & $\mathcal{O}\left(T_{\mathrm{OPA}} K^{3.5}\right)$ \\
\hline & APA & $\mathcal{O}\left(T_{\mathrm{APA}} M K^{2}\right)$ \\
\hline & UPA & $\mathcal{O}\left(M K^{2}\right)$ \\
\hline
\end{tabular}

\section{4}

\section{Numerical Results}

In this section, we add the RMMSE precoder to our experiments, to be compared to the $\mathrm{CB}$ and ZF precoders and most importantly, to the MMSE scheme. Note that all simulations consider imperfect CSI since under perfect CSI the RMMSE precoder converges to the MMSE precoder for cell-free.

The caption has the same notation of Chapter 3. We considered the following:

- Precoding + Power Allocation + APS

For each category, we have the following methods:

- Precoding: CB, ZF, MMSE and RMMSE

- Power Allocation: OPA and UPA

- APS: LS-APS

Before presenting our experiments, let's first recapitulate some parameters used in the simulations. The parameter $n$ is used to define the percentage of CSI imperfection $(0 \leq n \leq 1)$, building a relation between $\alpha_{m, k}$ and $\beta_{m, k}$ 
where $\alpha_{m, k}=n \beta_{m, k}, \alpha_{m, k}$ is the variance of $\hat{g}_{m, k}$ and $\beta_{m, k}$ is the LS coefficient. If we would like to consider $5 \%$ of CSI imperfection, for example, $n=0.95$. We also describe $E_{t r}$ as the average transmitted power, $M$ as the total number of antenna elements and $\rho_{f}$ as the maximum transmitted power of each antenna. All experiments are averaged over 120 channel realizations and assumed $\sigma_{s}^{2}=1$, where $\sigma_{s}^{2}$ is the symbol variance.

In the first experiment we compare the strategies in terms of BER vs. SNR, only with UPA. We assume imperfect CSI $(n=0.99)$, QPSK modulation and 100 symbols per packet. No APS scheme is considered. We analyse both single and multiple-antenna APs, in Fig. 4.1 and Fig. 4.2, respectively. In the first scenario we have $L=96$ APs with $N=1$ antennas each, and $K=8$ users. In contrast, for the multiple-antenna case we present the same total number of transmit antennas, but now with $L=24$ APs and $N=4$ antennas each.

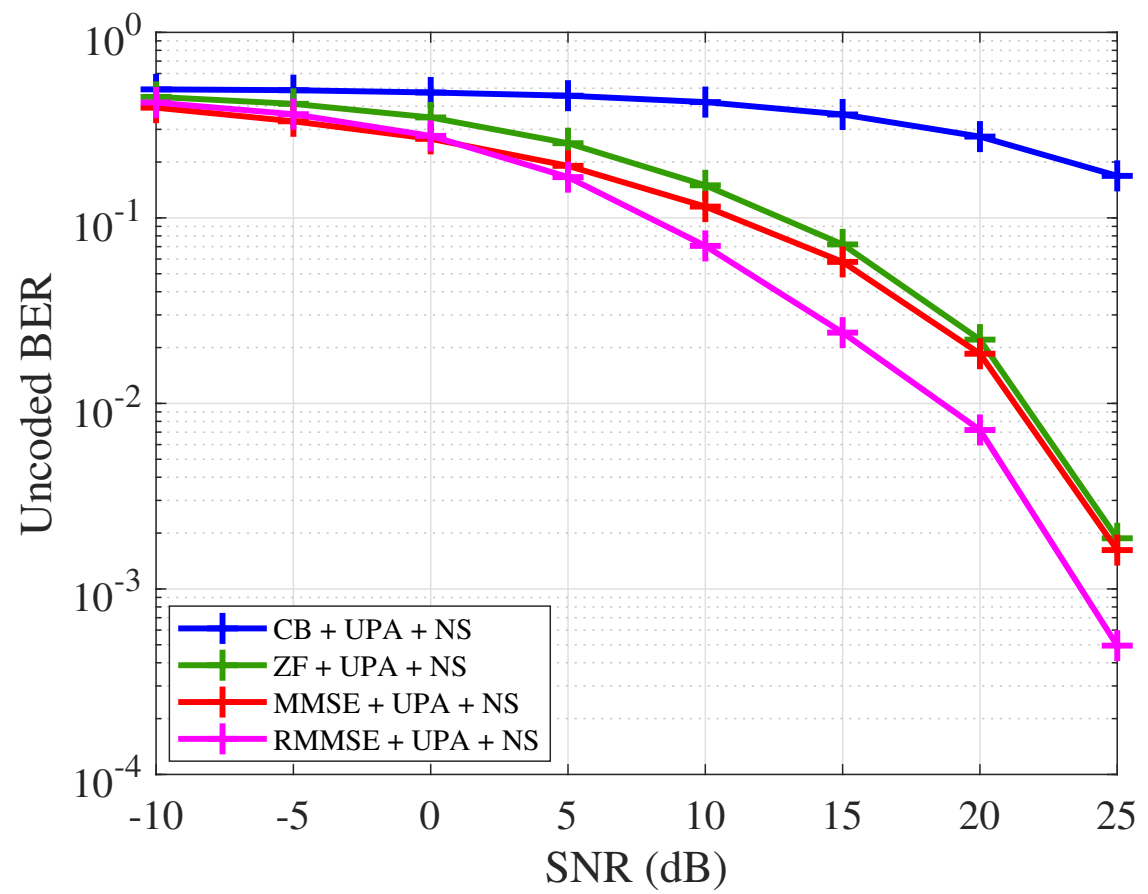

Figure 4.1: BER vs. SNR with $L=96, N=1, K=8, n=0.99,120$ channel realizations, 100 symbols per packet and $E_{t r}=M \rho_{f}$.

As we can observe, much lower bit error rates can be achieved in the single-antenna APs scenario. However, as mentioned before, it is much more expensive to install more APs than to add more antennas to an AP [41].

In Fig. 4.1 we can notice that in higher SNRs, for a same BER value, a difference of $2.2 \mathrm{~dB}$ can be achieved between RMMSE+UPA and MMSE+UPA, where RMMSE outperforms MMSE. The highest difference achieved is around $4 \mathrm{~dB}$. Between RMMSE+UPA and ZF+UPA, gains can go up to $5 \mathrm{~dB}$. 


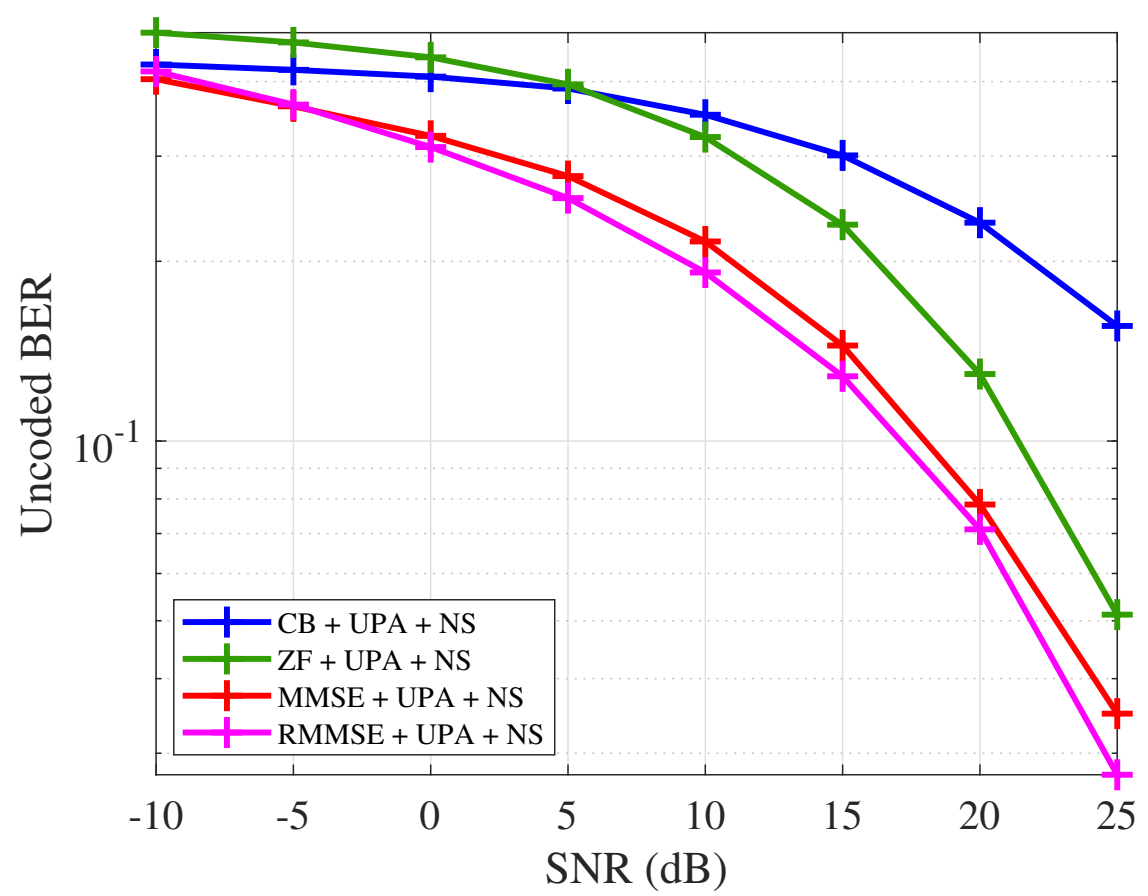

Figure 4.2: BER vs. SNR with $L=24, N=4, K=8, n=0.99,120$ channel realizations, 100 symbols per packet and $E_{t r}=M \rho_{f}$.

On the other hand, in Fig. 4.2, smaller differences are obtained between RMMSE+UPA and MMSE+UPA, up to $1.9 \mathrm{~dB}$, but greater improvements are seen between RMMSE+UPA and ZF+UPA, where differences can go from 3.2 to $13.5 \mathrm{~dB}$.

In Fig. 4.3 and Fig. 4.4, we maintain the UPA curves and add the OPA scheme to each of the precoding techniques.

When comparing RMMSE+UPA with RMMSE+OPA in Fig. 4.3, we remark differences up to $2.35 \mathrm{~dB}$, demonstrating the benefits of the OPA scheme. In addition, differences up to $2 \mathrm{~dB}$ can be obtained between RMMSE+OPA and MMSE+OPA in highest SNRs, for a same BER value. A bigger change can be observed between RMMSE+OPA and MMSE+UPA, where the discrepancy goes from $3 \mathrm{~dB}$ to $6 \mathrm{~dB}$. Compared to the $\mathrm{ZF}$ precoder, the RMMSE+OPA can outperform the ZF+OPA by about $3.38 \mathrm{~dB}$, closer to the highest SNR.

In Fig. 4.4, differences up to $3.9 \mathrm{~dB}$ are seen between RMMSE+UPA and RMMSE+OPA. Regarding RMMSE+OPA and MMSE+OPA, in lower SNRS, RMMSE+OPA outperforms the latter in $3 \mathrm{~dB}$ and in higher SNRs, is comparable to it. In the same way as the previous results, greater differences are seen between RMMSE+OPA and MMSE+UPA, where the gain can go up to $5.9 \mathrm{~dB}$. When compared to the $\mathrm{ZF}+\mathrm{OPA}$, the maximum difference is of 4.4 $\mathrm{dB}$ in favor of the RMMSE+OPA. 
Chapter 4. Iterative Robust MMSE Precoding and Power Allocation for Cell-Free Massive MIMO Systems

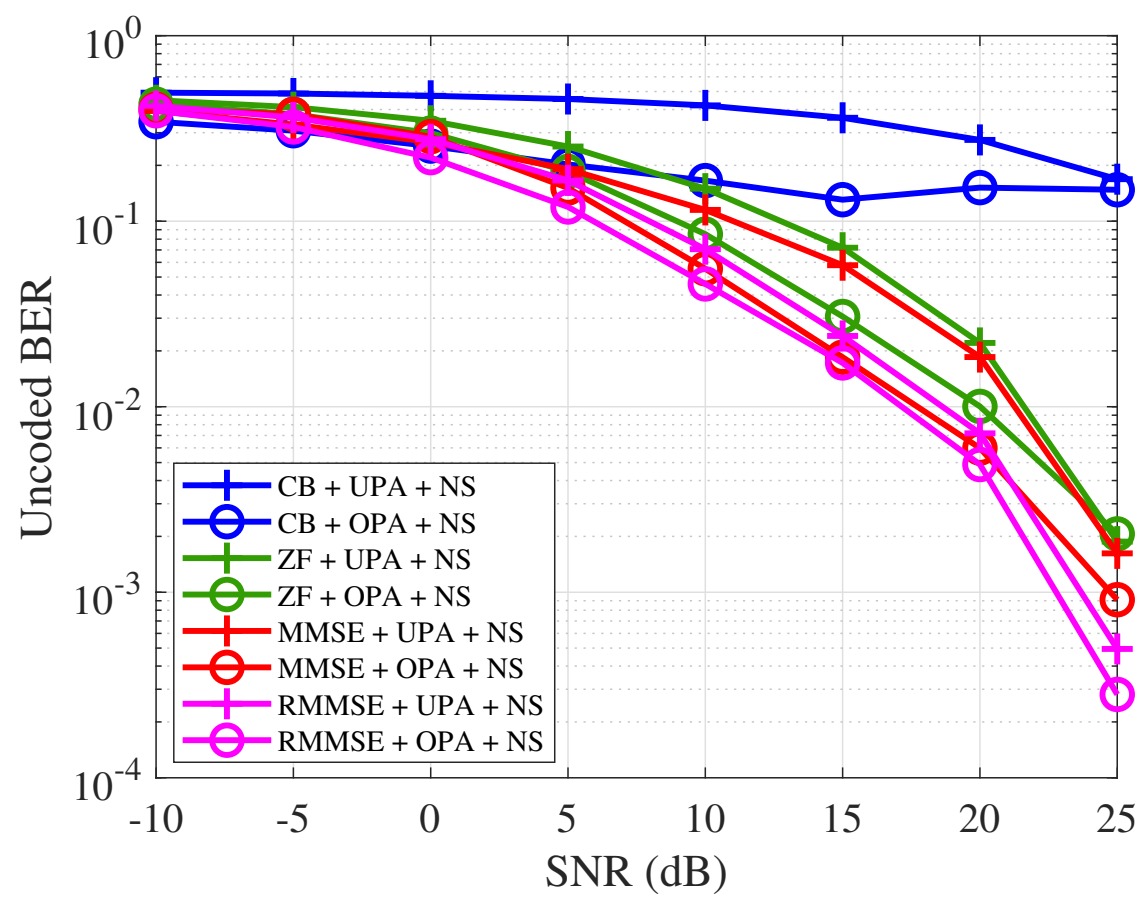

Figure 4.3: BER vs. SNR with $L=96, N=1, K=8, n=0.99,120$ channel realizations, 100 symbols per packet and $E_{t r}=M \rho_{f}$.

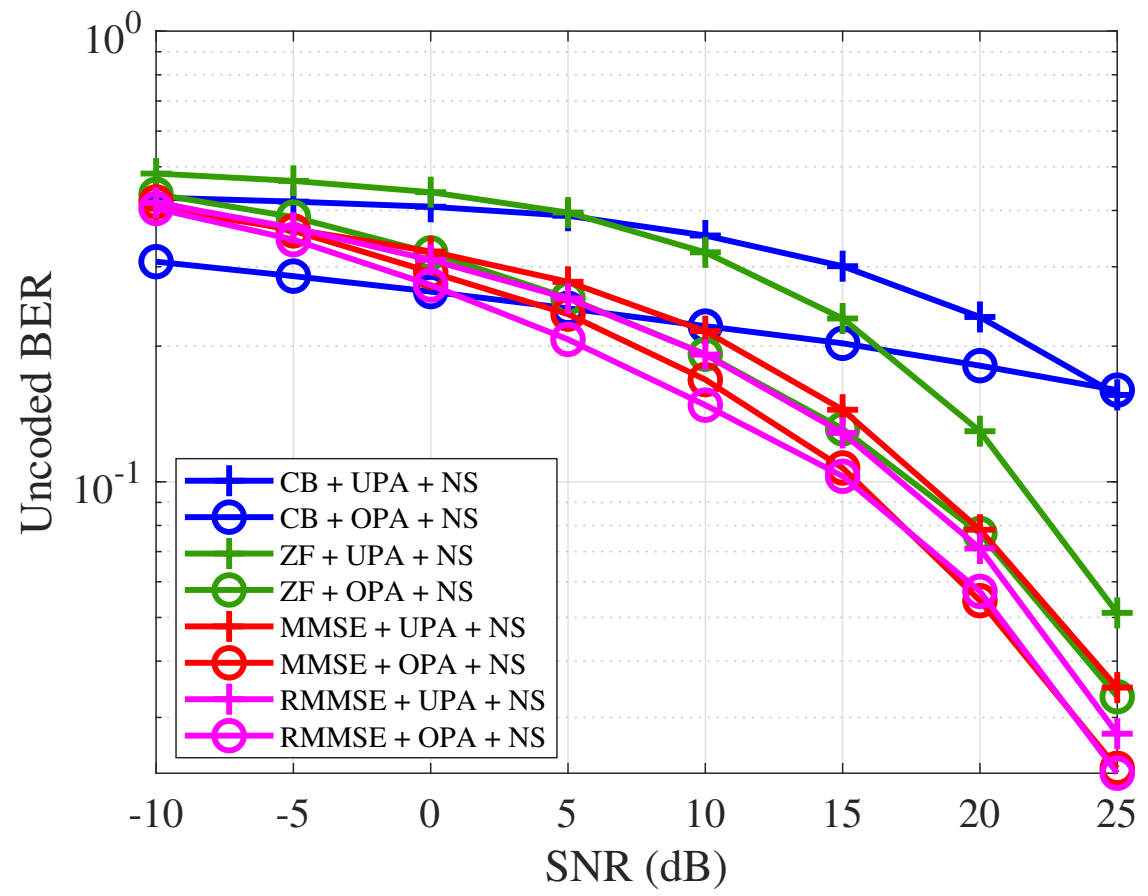

Figure 4.4: BER vs. SNR with $L=24, N=4, K=8, n=0.99,120$ channel realizations, 100 symbols per packet and $E_{t r}=M \rho_{f}$.

The last experiment in terms of BER vs. SNR describes the same system as before, but now with APS. The main objective is to decrease the number of selected APs by half, meaning that $S=L / 2$, without loosing much of the 
performance. In the single-antenna AP scenario, we have $L=96 \mathrm{APs}$ and $S=48$ selected ones. In the multiple-antenna case, we consider an antenna array of $N=4$ elements each, $L=24$ APs (total of $M=96$ antennas) and $S=12$ selected APs (total of 48 selected antennas).

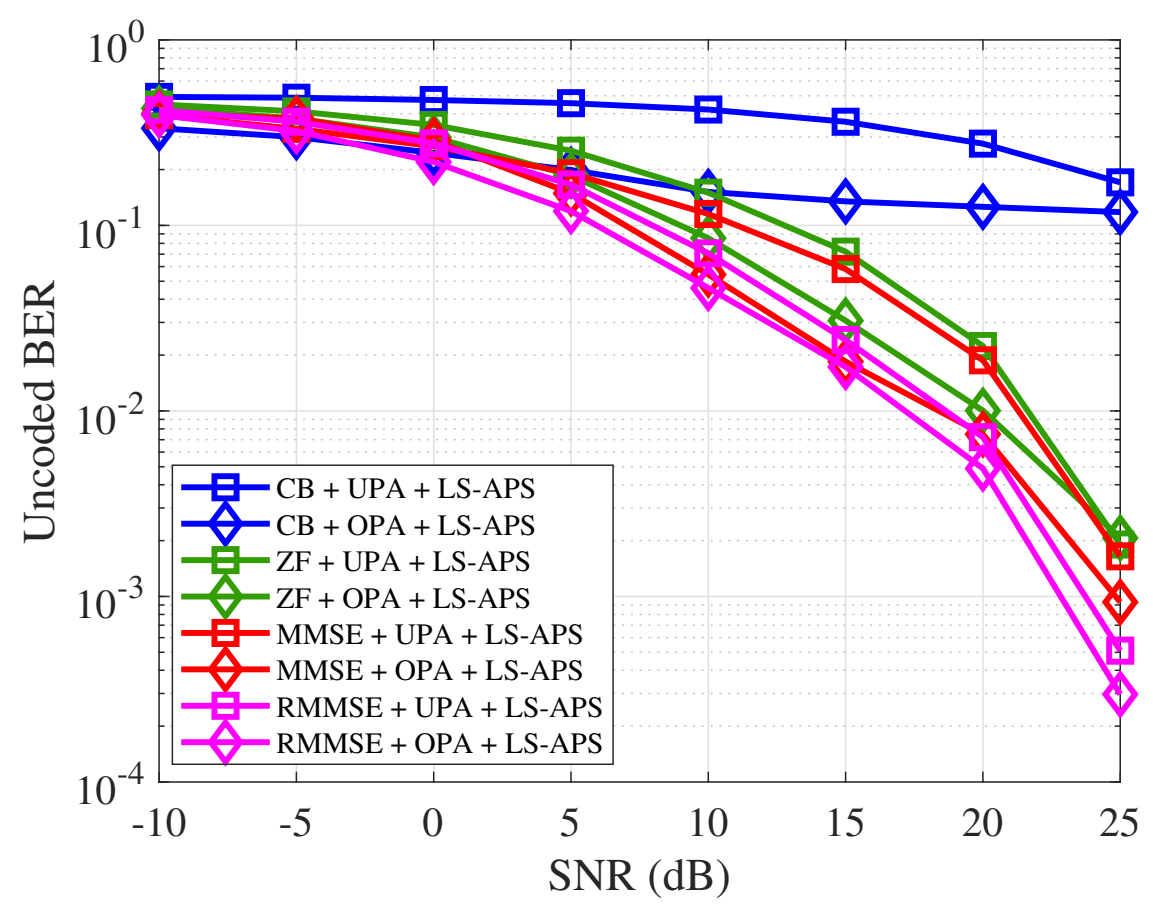

Figure 4.5: BER vs. SNR with $L=96, N=1, S=48, K=8, n=0.99,120$ channel realizations, 100 symbols per packet and $E_{t r}=M \rho_{f}$.

It is remarkable that Fig. 4.5 and Fig. 4.3 are extremely similar. This is due to the fact that APS makes almost no modifications in the performance of the precoding and power allocation techniques. The greatest change that can be seen is in RMMSE+OPA for $\mathrm{SNR}=25 \mathrm{~dB}$, where the difference was only of $6 \%$, between the NS and the LS-APS scheme.

Likewise, in Fig. 4.6 and Fig. 4.4, the same can be observed. Even in a multiple-antenna APs system, APS does not deteriorate the performance. The biggest change happens in MMSE+UPA, where a difference of $10 \%$ can be obtained when comparing with and without APS.

The next experiment is to compare the analysed techniques in terms of sum-rate vs. SNR. In the subsequent tests, the CSI imperfection is raised to $10 \%$, meaning that $n=0.9$. Moreover, we enlarged the system, now assuming $L=128$ APs, $N=1$ antenna elements each and $K=16$ users. 


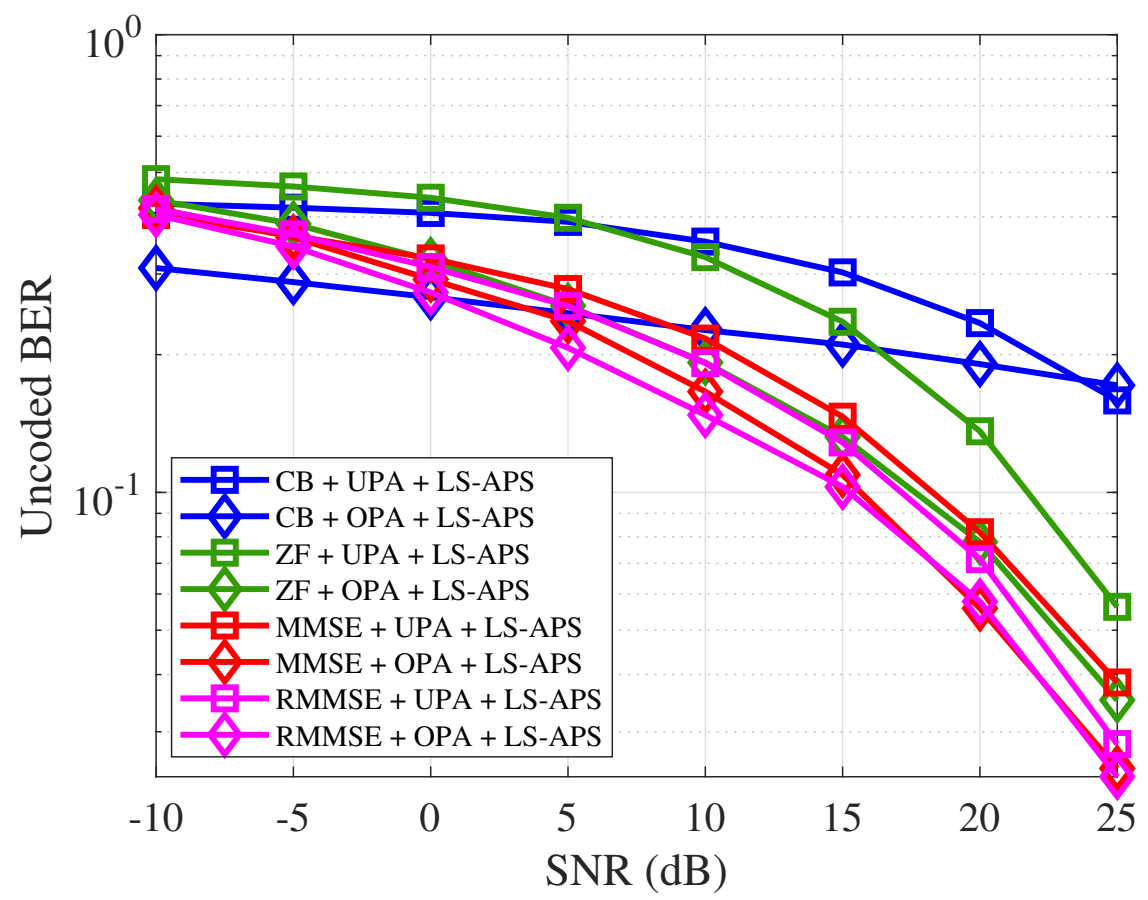

Figure 4.6: BER vs. SNR with $L=24, N=4, S=12, K=8, n=0.99,120$ channel realizations, 100 symbols per packet and $E_{t r}=M \rho_{f}$.

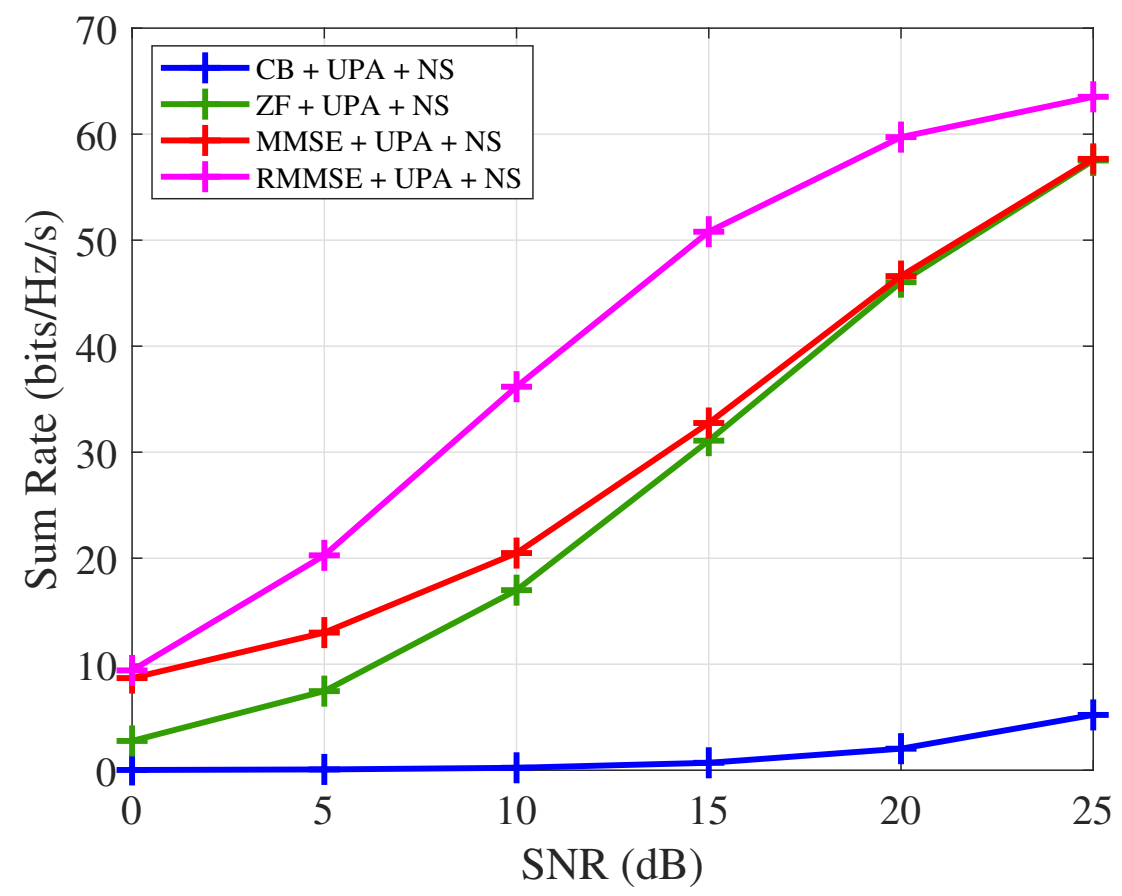

Figure 4.7: Sum-Rate vs. SNR with $L=128, \mathrm{~N}=1, K=16, n=0.9,120$ channel realizations and $E_{t r}=M \rho_{f}$.

What can be concluded from Fig. 4.7 is that the RMMSE precoder is more successful than all other precoders, when UPA is applied. In $10 \mathrm{~dB}$, for example, a gain of more than $70 \%$ can be experienced by the RMMSE+UPA in 
comparison with MMSE+UPA. Regarding the ZF+UPA, in the same SNR, the difference between it and the RMMSE+UPA is of more than $110 \%$. A partial conclusion that can be taken from this is that, with higher CSI imperfection, the discrepancy between RMMSE and the other precoders is increased, since they are not designed to mitigate the effects of channel estimation error.

In Fig. 4.8, the precoders with UPA techniques are kept and compared with the same precoding schemes, combined with OPA.

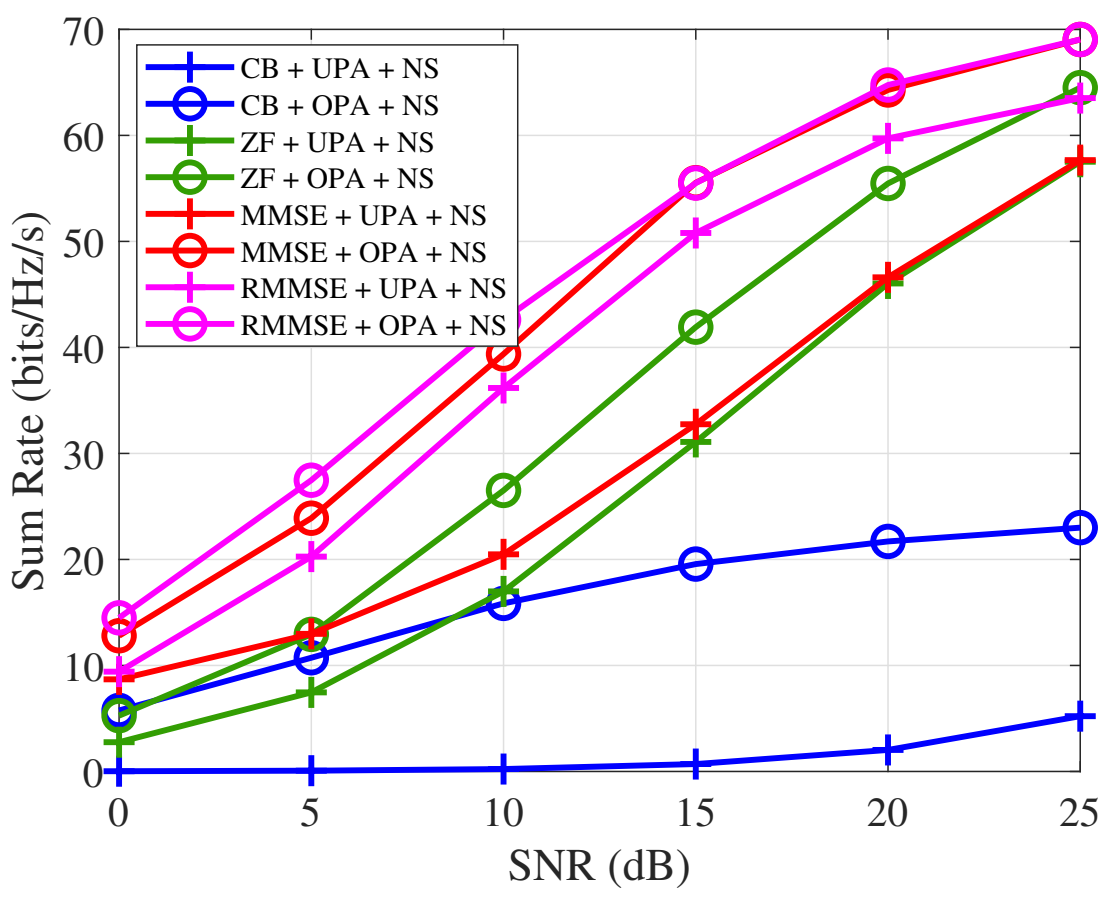

Figure 4.8: Sum-Rate vs. SNR with $L=128, \mathrm{~N}=1, K=16, n=0.9,120$ channel realizations and $E_{t r}=M \rho_{f}$.

When OPA is applied to the precoding techniques, all rates are improved, compared to the uniform schemes. Between RMMSE+OPA and RMMSE+UPA we can see a gain of $53 \%$ in SNR $=0 \mathrm{~dB}$, for example. Smaller gains are observed between RMMSE+OPA and MMSE+OPA where the maximum achieved is around $15 \%$ in $5 \mathrm{~dB}$. Greater improvements can be seen between RMMSE+OPA and MMSE+UPA where the gain goes around $110 \%$ in $\mathrm{SNR}=5 \mathrm{~dB}$. Finally, between RMMSE $+\mathrm{OPA}$ and $\mathrm{ZF}+\mathrm{OPA}$, the gain can be up to $175 \%$, for example, in $\mathrm{SNR}=0 \mathrm{~dB}$. 
The last figure we would like to illustrate in terms of sum-rate vs. SNR is about the APS technique being applied to the previous system. As in the first APS experiment presented in this chapter, shown in Fig. 4.5 and Fig. 4.6, we would like to reduce the computational complexity and decrease the number of selected APs in 50\%. In the results below, we consider single-antenna APs scenario with $L=128$ APs and $S=64$ selected ones.

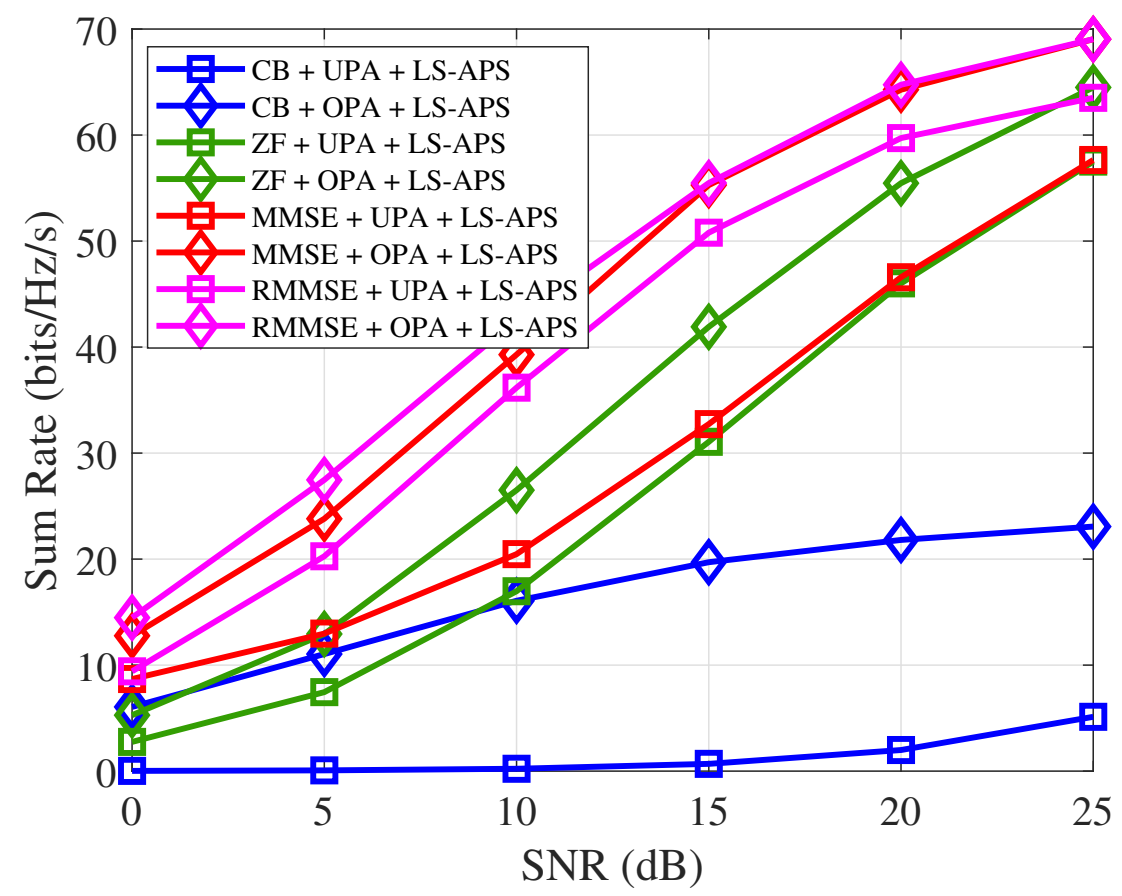

Figure 4.9: Sum-Rate vs. SNR with $L=128, \mathrm{~N}=1, S=64, K=16, n=0.9$, 120 channel realizations and $E_{t r}=M \rho_{f}$.

The insight provided by Fig. 4.9 is the same as provided by Fig. 4.5 and Fig. 4.6. When we compare the performance obtained in a NS scheme (Fig. 4.8) with LS-APS (Fig. 4.9), almost no change can be observed. The highest improvement happens in $\mathrm{CB}+\mathrm{OPA}$, where the LS-APS technique provides a gain of $5 \%$ in $0 \mathrm{~dB}$. For the RMMSE with OPA and UPA, no gain or loss can be perceived. In a general way, changes between NS and LS-APS are of less than $1 \%$.

In the last experiment of this chapter, we compare the investigated strategies in terms of Minimum SINR vs. SNR, in order to attest the effectiveness of the OPA algorithm. Here, we only investigate the MMSE and RMMSE techniques and take APS into account. 


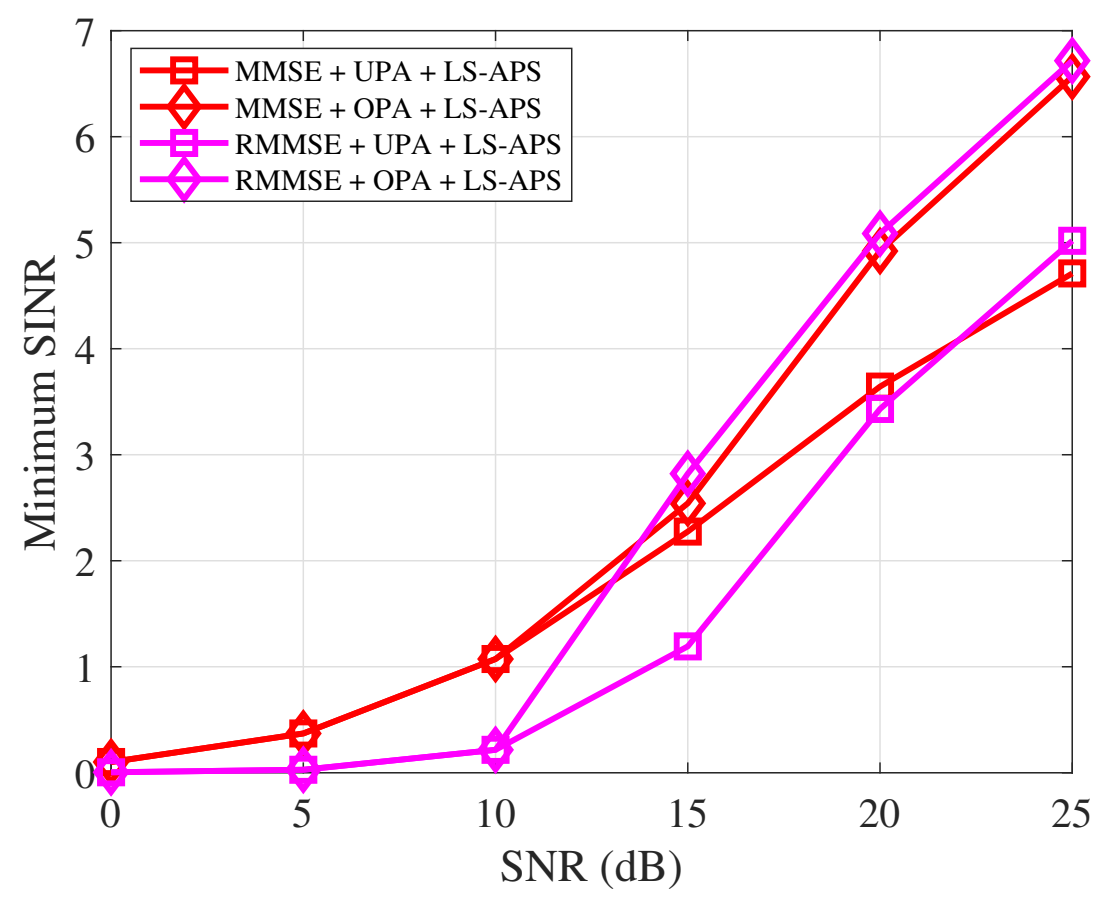

Figure 4.10: Minimum SINR vs. SNR with $L=128, \mathrm{~N}=1, S=64, K=16$, $n=0.9,120$ channel realizations and $E_{t r}=M \rho_{f}$.

As we can see in Fig. 4.10, for both MMSE and RMMSE techniques, the minimum SINRs of the OPA strategies have at least the same performance of the UPA ones, which is what we were aiming for. Although in low SNR values the MMSE outperforms RMMSE for both power allocation techniques, from $15 \mathrm{~dB}, \mathrm{RMMSE}+\mathrm{OPA}$ presents an improved performance when compared to MMSE+OPA.

As highlighted in the last chapter, the choice of whether to improve the minimum SINR or the sum-rate is a decision taken in the project. By adjusting the parameters in the bisection method (Algorithm 1), $t_{b}$ and $t_{e}$, both can be achieved, but not simultaneously, in accordance with the challenges faced by max-min problems.

\section{5}

\section{Summary}

In this part, we have developed an iterative RMMSE precoder with total power constraint. Two power allocation techniques taking into account the RMMSE precoding scheme have been derived. A sum-rate analysis of the proposed strategy has been carried out and its computational cost, evaluated. Numerical results show that the robust alternative outperforms previous ones in the presence of imperfect CSI. Furthermore, the technique is not affected by APS strategies and presents comparable computational complexity. 


\section{5}

\section{Conclusion and Future Work}

In this thesis, we developed APS, precoding and power allocation techniques for Cell-Free Massive MIMO Systems. First, we proposed two APS schemes, one based on ES and a suboptimal solution, based on the LS coefficients. We derive an iterative precoder with MMSE criteria, considering total power constraint. Power allocation schemes are also presented, with optimal, adaptive and uniform solutions. Then, we extend the precoding technique to a robust version, where total power constraint is also taken into account. Both optimal and uniform power allocation techniques based on the robust precoding are created. We have also developed a sum-rate analysis of the proposed schemes, as well as an evaluation of their computational complexity, to be compared with the cost of existing strategies. Numerical results put in evidence the potential of the developed solutions, outperforming $\mathrm{CB}$ and $\mathrm{ZF}$ precoder in both UPA and OPA schemes and maintaining performance when APS is applied.

In Chapter 2 we summarized the ideas and works that have lead to the creation of Cell-Free Massive MIMO Systems. Topics such as massive MIMO, DAS and network MIMO are covered. Moreover, we provided an insight on the most important precoding, power allocation and APS techniques, as well as robust solutions, that can be applied in these scenarios to improve performance and mitigate interference and channel estimation errors. The system model is also presented, together with channel estimation and downlink payload data transmission. Simulations show the benefits of DAS, cooperative networks and cell-free systems, when compared to CAS.

In Chapter 3, two APS techniques are developed. The first is based on ES, testing all possibilities of arrangements, given a fixed number $S$ of selected APs. The second is based on the LS coefficients, where they are put in a descending order and the largest ones are chosen, also respecting the limit of $S$ selected APs. The chosen configurations are applied to the CSI matrix, the CSI error matrix and their respective variances. Next, an MMSE precoding scheme is derived, with total power constraint. Power allocation methods are proposed as well, with optimal, adaptive and uniform approaches. The OPA scheme is configured as a max-min fairness power allocation problem, where 
we aim to maximize the minimum SINR among all users. On the other hand, APA is defined as a SG learning algorithm where we take into consideration the gradient of the MSE. Then, UPA is simply described as a technique where all power coefficients $\left(\eta_{k}\right)$ are equal. All resource allocation methods must fulfil the per-antenna power constraint. A sum-rate analysis is made based on the introduced techniques and their computational complexity is calculated. In the end, numerical results are illustrated, testifying that the MMSE precoder has improved performance when compared to $\mathrm{CB}$ and $\mathrm{ZF}$, in terms of BER, sumrate and minimum SINR. In all precoding schemes OPA performs better than APA and UPA, and APA can surpass UPA in terms of BER and sum-rate. In a small system, ES-APS can be more successful or at least maintain performance when compared to a NS scheme and LS-APS can sustain performance in larger systems.

A robust version of the precoding schemes is presented in Chapter 4, where the total power constraint is considered. The presented technique has almost the same design from the one in Chapter 3, but now, is projected to outperform previous ones in the presence of imperfect CSI. Respective power allocation methods are developed to fit the robustness design, in the same fashion as before, with OPA and UPA. A sum-rate analysis is derived, as well as the calculation of the computational cost of each strategy. Numerical results and discussions are shown in the end of the chapter proving that the robust strategy outperforms standard approaches when we consider imperfect CSI.

Most of the techniques proposed in this thesis are centralized and carried out at the CPU. As a result, the solutions lack an appropriate analysis of the system's scalability. For this reason, in the future, a fully distributed version of the developed schemes can be examined, in terms of precoding and power control, as a means to improve the scalability of such networks.

Another possibility to continue this work is to analyse an equivalent version on the uplink channel. All the techniques presented here are practical and can be derived and adapted to the uplink, due to the TDD protocol, which provides channel reciprocity. Detection techniques can be also incorporated to successive interference cancellation (SIC), in order to facilitate the demodulation of signals affected by interference and increase capacity [62].

Finally, for the downlink, precoding schemes that are variants or extensions from the analysed precoders can be developed, such as an MMSE precoder with per-antenna power constraint, a robust MMSE precoder with per-antenna power constraint or a robust ZF precoder. Furthermore, other precoding strategies can be applied to these systems, such as THP, which has never been used before in cell-free networks. 


\section{Bibliography}

[1] STEPHEN BOYD, L. V.. Convex Optimization. Cambridge University Press, 2019.

[2] NGO, H. Q.; TRAN, L.-N.; DUONG, T. Q.; MATTHAIOU, M. ; LARSSON, E. G.. On the total energy efficiency of cell-free massive MIMO. IEEE Transactions on Green Communications and Networking, 2(1):25-39, mar 2018.

[3] MARZETTA, T. L.; LARSSON, E. G.; YANG, H. ; NGO, H. Q.. Fundamentals of Massive MIMO. Cambridge University Press, nov 2016.

[4] HEATH, R.; PETERS, S.; WANG, Y. ; ZHANG, J.. A current perspective on distributed antenna systems for the downlink of cellular systems. IEEE Communications Magazine, 51(4):161-167, apr 2013.

[5] DAI, L.. A comparative study on uplink sum capacity with colocated and distributed antennas. IEEE Journal on Selected Areas in Communications, 29(6):1200-1213, jun 2011.

[6] KARAKAYALI, M.; FOSCHINI, G. ; VALENZUELA, R.. Network coordination for spectrally efficient communications in cellular systems. IEEE Wireless Communications, 13(4):56-61, aug 2006.

[7] FENG, W.; WANG, Y.; GE, N.; LU, J. ; ZHANG, J.. Virtual MIMO in multi-cell distributed antenna systems: Coordinated transmissions with large-scale CSIT. IEEE Journal on Selected Areas in Communications, 31(10):2067-2081, oct 2013.

[8] NGO, H. Q.; ASHIKHMIN, A.; YANG, H.; LARSSON, E. G. ; MARZETTA, T. L.. Cell-free massive MIMO versus small cells. IEEE Transactions on Wireless Communications, 16(3):1834-1850, mar 2017.

[9] NAYEBI, E.; ASHIKHMIN, A.; MARZETTA, T. L.; YANG, H. ; RAO, B. D. Precoding and power optimization in cell-free massive MIMO systems. IEEE Transactions on Wireless Communications, 16(7):4445-4459, jul 2017. 
[10] BJORNSON, E.; SANGUINETTI, L.. Making cell-free massive MIMO competitive with MMSE processing and centralized implementation. IEEE Transactions on Wireless Communications, 19(1):77-90, jan 2020.

[11] CAI, Y.; DE LAMARE, R. C.; YANG, L.-L. ; ZHAO, M.. Robust MMSE precoding based on switched relaying and side information for multiuser MIMO relay systems. IEEE Transactions on Vehicular Technology, 64(12):5677-5687, dec 2015.

[12] ZHANG, B.; HE, Z.; NIU, K. ; ZHANG, L.. Robust linear beamforming for MIMO relay broadcast channel with limited feedback. IEEE Signal Processing Letters, 17(2):209-212, feb 2010.

[13] KHALED, N.; LEUS, G.; DESSET, C. ; MAN, H. D.. A robust joint linear precoder and decoder MMSE design for slowly time-varying MIMO channels. In: 2004 IEEE INTERNATIONAL CONFERENCE ON ACOUSTICS, SPEECH, AND SIGNAL PROCESSING. IEEE, may 2004.

[14] WANG, J.; BENGTSSON, M.. Joint optimization of the worst-case robust MMSE MIMO transceiver. IEEE Signal Processing Letters, 18(5):295-298, may 2011.

[15] GUO, Y.; LEVY, B.. Worst-case MSE precoder design for imperfectly known MIMO communications channels. IEEE Transactions on Signal Processing, 53(8):2918-2930, aug 2005.

[16] ANDREWS, J. G.; BUZZI, S.; CHOI, W.; HANLY, S. V.; LOZANO, A.; SOONG, A. C. K. ; ZHANG, J. C.. What will 5G be? IEEE Journal on Selected Areas in Communications, 32(6):1065-1082, jun 2014.

[17] MARZETTA, T. L.. Noncooperative cellular wireless with unlimited numbers of base station antennas. IEEE Transactions on Wireless Communications, 9(11):3590-3600, nov 2010.

[18] BOCCARDI, F.; HEATH, R. W.; LOZANO, A.; MARZETTA, T. L. ; POPOVSKI, P.. Five disruptive technology directions for 5G. IEEE Communications Magazine, 52(2):74-80, feb 2014.

[19] LARSSON, E. G.; EDFORS, O.; TUFVESSON, F. ; MARZETTA, T. L.. Massive MIMO for next generation wireless systems. IEEE Communications Magazine, 52(2):186-195, feb 2014. 
[20] LU, L.; LI, G. Y.; SWINDLEHURST, A. L.; ASHIKHMIN, A. ; ZHANG, R.. An overview of massive MIMO: Benefits and challenges. IEEE Journal of Selected Topics in Signal Processing, 8(5):742-758, oct 2014.

[21] SHAFI, M.; MOLISCH, A. F.; SMITH, P. J.; HAUSTEIN, T.; ZHU, P.; SILVA, P. D.; TUFVESSON, F.; BENJEBBOUR, A. ; WUNDER, G.. 5G: A tutorial overview of standards, trials, challenges, deployment, and practice. IEEE Journal on Selected Areas in Communications, 35(6):12011221, jun 2017.

[22] GIORDANO, L. G.; GARCIA-RODRIGUEZ, A.; HO, L. ; LOPEZ-PEREZ, D.. Next generation Wi-Fi: Deployment guidelines and benefits of massive MIMO for the enterprise. In: 2020 IEEE 91ST VEHICULAR TECHNOLOGY CONFERENCE (VTC2020-SPRING). IEEE, may 2020.

[23] CHOI, W.; ANDREWS, J.. Downlink performance and capacity of distributed antenna systems in a multicell environment. IEEE Transactions on Wireless Communications, 6(1):69-73, jan 2007.

[24] ZHU, H.; WANG, J.. Radio resource allocation in multiuser distributed antenna systems. IEEE Journal on Selected Areas in Communications, 31(10):2058-2066, oct 2013.

[25] LIU, Z.; DAI, L.. A comparative study of downlink MIMO cellular networks with co-located and distributed base-station antennas. IEEE Transactions on Wireless Communications, 13(11):6259-6274, nov 2014.

[26] GESBERT, D.; HANLY, S.; HUANG, H.; SHITZ, S. S.; SIMEONE, O. ; YU, W.. Multi-cell MIMO cooperative networks: A new look at interference. IEEE Journal on Selected Areas in Communications, 28(9):1380-1408, dec 2010.

[27] MARSCH, P.; FETTWEIS, G.. Uplink CoMP under a constrained backhaul and imperfect channel knowledge. IEEE Transactions on Wireless Communications, 10(6):1730-1742, jun 2011.

[28] DAI, H.; MOLISCH, A. ; POOR, H.. Downlink capacity of interferencelimited MIMO systems with joint detection. IEEE Transactions on Wireless Communications, 3(2):442-453, mar 2004.

[29] LI, P.; DE LAMARE, R. C.. Distributed iterative detection with reduced message passing for networked MIMO cellular systems. IEEE Transactions on Vehicular Technology, 63(6):2947-2954, jul 2014. 
[30] IRMER, R.; DROSTE, H.; MARSCH, P.; GRIEGER, M.; FETTWEIS, G.; BRUECK, S.; MAYER, H.-P.; THIELE, L. ; JUNGNICKEL, V.. Coordinated multipoint: Concepts, performance, and field trial results. IEEE Communications Magazine, 49(2):102-111, feb 2011.

[31] LU, X.; NI, Q.; LI, W. ; ZHANG, H.. Dynamic user grouping and joint resource allocation with multi-cell cooperation for uplink virtual MIMO systems. IEEE Transactions on Wireless Communications, 16(6):3854-3869, jun 2017.

[32] TRUONG, K. T.; HEATH, R. W.. The viability of distributed antennas for massive MIMO systems. In: 2013 ASILOMAR CONFERENCE ON SIGNALS, SYSTEMS AND COMPUTERS. IEEE, nov 2013.

[33] JOUNG, J.; CHIA, Y. K. ; SUN, S.. Energy-efficient, large-scale distributed-antenna system (L-DAS) for multiple users. IEEE Journal of Selected Topics in Signal Processing, 8(5):954-965, oct 2014.

[34] NGO, H. Q.; ASHIKHMIN, A.; YANG, H.; LARSSON, E. G. ; MARZETTA, T. L.. Cell-free massive MIMO: Uniformly great service for everyone. In: 2015 IEEE 16TH INTERNATIONAL WORKSHOP ON SIGNAL PROCESSING ADVANCES IN WIRELESS COMMUNICATIONS (SPAWC). IEEE, jun 2015.

[35] YANG, H.; MARZETTA, T. L.. Energy efficiency of massive MIMO: Cell-free vs. cellular. In: 2018 IEEE 87TH VEHICULAR TECHNOLOGY CONFERENCE (VTC SPRING). IEEE, jun 2018.

[36] NAYEBI, E.; ASHIKHMIN, A.; MARZetTA, T. L. ; RAO, B. D.. Performance of cell-free massive MIMO systems with MMSE and LSFD receivers. In: 2016 50TH ASILOMAR CONFERENCE ON SIGNALS, SYSTEMS AND COMPUTERS. IEEE, nov 2016.

[37] NGO, H. Q.; TRAN, L.-N.; DUONG, T. Q.; MATTHAIOU, M. ; LARSSON, E. G. Energy efficiency optimization for cell-free massive MIMO. In: 2017 IEEE 18TH INTERNATIONAL WORKSHOP ON SIGNAL PROCESSING ADVANCES IN WIRELESS COMMUNICATIONS (SPAWC). IEEE, jul 2017.

[38] NGUYEN, L. D.; DUONG, T. Q.; NGO, H. Q. ; TOURKI, K.. Energy efficiency in cell-free massive MIMO with zero-forcing precoding design. IEEE Communications Letters, 21(8):1871-1874, aug 2017. 
[39] BASHAR, M.; CUMANAN, K.; BURR, A. G.; DEBBAH, M. ; NGO, H. Q.. On the uplink max-min SINR of cell-free massive MIMO systems. IEEE Transactions on Wireless Communications, 18(4):2021-2036, apr 2019.

[40] CHEN, Z.; BJORNSON, E.. Channel hardening and favorable propagation in cell-free massive MIMO with stochastic geometry. IEEE Transactions on Communications, 66(11):5205-5219, nov 2018.

[41] IBRAHIM, A. A. I.; ASHIKHMIN, A.; MARZETTA, T. L. ; LOVE, D. J.. Cell-free massive MIMO systems utilizing multi-antenna access points. In: 2017 51ST ASILOMAR CONFERENCE ON SIGNALS, SYSTEMS, AND COMPUTERS. IEEE, oct 2017.

[42] LI, J.; STOICA, P. ; WANG, Z.. On robust capon beamforming and diagonal loading. IEEE Transactions on Signal Processing, 51(7):17021715, jul 2003.

[43] ELNASHAR, A.; ELNOUBI, S. M. ; EL-MIKATI, H. A.. Further study on robust adaptive beamforming with optimum diagonal loading. IEEE Transactions on Antennas and Propagation, 54(12):3647-3658, dec 2006.

[44] YU, L.; FAN, Y.; WEI, Y. ; XU, R.. Robust adaptive beamforming method for large-scale array with automatic diagonal loading and steering vector estimation. The Journal of Engineering, 2019(21):8047-8050, nov 2019.

[45] BESSON, O.; VINCENT, F.. Performance analysis of beamformers using generalized loading of the covariance matrix in the presence of random steering vector errors. IEEE Transactions on Signal Processing, 53(2):452-459, feb 2005.

[46] WANG, J.; BENGTSSON, M.; OTTERSTEN, B. ; ANIEL P. PALOMAR. Robust MIMO precoding for several classes of channel uncertainty. IEEE Transactions on Signal Processing, 61(12):3056-3070, jun 2013.

[47] TAJER, A.; PRASAD, N. ; WANG, X.. Robust linear precoder design for multi-cell downlink transmission. IEEE Transactions on Signal Processing, 59(1):235-251, jan 2011.

[48] SHEN, C.; CHANG, T.-H.; WANG, K.-Y.; QIU, Z. ; CHI, C.-Y.. Distributed robust multicell coordinated beamforming with imper- 
fect CSI: An ADMM approach. IEEE Transactions on Signal Processing, 60(6):2988-3003, jun 2012.

[49] RUAN, H.; DE LAMARE, R. C.. Robust adaptive beamforming based on low-rank and cross-correlation techniques. IEEE Transactions on Signal Processing, 64(15):3919-3932, aug 2016.

[50] RUAN, H.; DE LAMARE, R. C.. Distributed robust beamforming based on low-rank and cross-correlation techniques: Design and analysis. IEEE Transactions on Signal Processing, 67(24):6411-6423, dec 2019.

[51] SHEN, H.; WANG, J.; XU, W.; RONG, Y. ; ZHAO, C.. A worst-case robust MMSE transceiver design for nonregenerative MIMO relaying. IEEE Transactions on Wireless Communications, 13(2):695-709, feb 2014.

[52] ZHANG, L.; CAI, Y.; DE LAMARE, R. C. ; ZHAO, M.. Robust multibranch tomlinson-harashima precoding design in amplify-andforward MIMO relay systems. IEEE Transactions on Communications, 62(10):3476-3490, oct 2014.

[53] TANG, A.; SUN, J. ; GONG, K.. Mobile propagation loss with a low base station antenna for NLOS street microcells in urban area. In: IEEE VTS 53RD VEHICULAR TECHNOLOGY CONFERENCE, SPRING 2001. PROCEEDINGS (CAT. NO.01CH37202). IEEE, may 2001.

[54] BROWN, T.; CARVALHO, E. D. ; KYRITSI, P.. Practical Guide to the MIMO Radio Channel with MATLAB® Examples. John Wiley \& Sons, Ltd, feb 2012.

[55] TSE, D.; VISWANATH, P.. Fundamentals of Wireless Communication. Cambridge University Press, may 2005.

[56] JOHAM, M.; UTSCHICK, W. ; NOSSEK, J.. Linear transmit processing in MIMO communications systems. IEEE Transactions on Signal Processing, 53(8):2700-2712, aug 2005.

[57] ZHANG, J.; CHEN, S.; LIN, Y.; ZHENG, J.; AI, B. ; HANZO, L.. Cell-free massive MIMO: A new next-generation paradigm. IEEE Access, 7:99878-99888, 2019.

[58] CLARKE, P.; DE LAMARE, R. C.. Transmit diversity and relay selection algorithms for multirelay cooperative MIMO systems. IEEE Transactions on Vehicular Technology, 61(3):1084-1098, mar 2012. 
[59] HAYES, M. H.. Statistical Digital Signal Processing and Modeling. John Wiley \& Sons, Ltd, apr 1996.

[60] HAYKIN, S.. Adaptive Filter Theory. Pearson, jun 2013.

[61] WATKINS, D. S.. Fundamentals of Matrix Computations. Wiley, 2014.

[62] FA, R.; DE LAMARE, R.. Multi-branch successive interference cancellation for MIMO spatial multiplexing systems: design, analysis and adaptive implementation. IET Communications, 5(4):484-494, mar 2011.

[63] SCHARF, L. L.. Statistical Signal Processing: Detection, Estimation, and Time Series Analysis. Pearson, jul 1991.

[64] FUKUDA, R. M.; ABRAO, T.. Linear, quadratic, and semidefinite programming massive MIMO detectors: Reliability and complexity. IEEE Access, 7:29506-29519, 2019. 
A

\section{Computational Complexity}

\section{A.1}

\section{APS Complexity}

\section{ES-APS}

- Permutation of a string $=\mathcal{O}(L !)$

- Permutation of vectors $=\mathcal{O}(K !)$

Overall Complexity: $\mathcal{O}(L !)+\mathcal{O}(K !)=\mathcal{O}(L !)$, considering $L>K$

\section{LS-APS}

- Quick sort $=\mathcal{O}\left(M^{2}\right)$

- Element-wise multiplication of $\mathbf{Q}$ by $\boldsymbol{\alpha}, \boldsymbol{\beta}, \hat{\mathbf{G}}$ and $\tilde{\mathbf{G}}=4 M K$

Overall Complexity: $\mathcal{O}\left(M^{2}\right)+\mathcal{O}(M K)=\mathcal{O}\left(M^{2}\right)$

\section{A. 2}

Precoders

\section{MMSE Precoder}

with

$$
\mathbf{P}_{\mathrm{MMSE}}=\frac{f_{\mathrm{MMSE}}}{\sqrt{\rho_{f}}}\left(\hat{\mathbf{G}}^{*} \hat{\mathbf{G}}^{T}+\frac{\operatorname{tr}\left(\mathbf{C}_{\mathbf{w}}\right)}{E_{t r}} \mathbf{I}\right)^{-1} \hat{\mathbf{G}}^{*} \mathbf{N}^{-1}
$$

$$
f_{\mathrm{MMSE}}=\sqrt{\frac{E_{t r}}{\operatorname{tr}\left(\tilde{\mathbf{P}} \mathbf{C}_{\mathbf{s}} \tilde{\mathbf{P}}^{H}\right)}} \text {. }
$$


$\tilde{\mathbf{P}} \mathbf{C}_{\mathbf{s}} \rightarrow M K^{2}$ multiplications $+M K(K-1)$ additions.

$\tilde{\mathbf{P}} \mathbf{C}_{\mathbf{s}} \tilde{\mathbf{P}}^{H} \rightarrow M^{2} K$ multiplications $+M^{2}(K-1)$ additions.

$\frac{E_{t r}}{\operatorname{tr}\left(\tilde{\mathbf{P}} \mathbf{C}_{\mathbf{s}} \tilde{\mathbf{P}}^{H}\right)} \rightarrow 1$ division $+(M-1)$ additions.

$\sqrt{\frac{E_{t r}}{\operatorname{tr}\left(\tilde{\mathbf{P}} \mathbf{C}_{\mathbf{s}} \tilde{\mathbf{P}}^{H}\right)}} \rightarrow 1$ square root

$\hat{\mathbf{G}}^{*} \hat{\mathbf{G}}^{T} \rightarrow M^{2} K$ multiplications $+M^{2}(K-1)$ additions.

$\frac{\operatorname{tr}\left(\mathbf{C}_{\mathbf{w}}\right)}{E_{t r}} \mathbf{I} \rightarrow 1$ division $+M^{2}$ multiplications $+(K-1)$ additions.

$\hat{\mathbf{G}}^{*} \hat{\mathbf{G}}^{T}+\frac{\operatorname{tr}\left(\mathbf{C}_{\mathbf{w}}\right)}{E_{t r}} \mathbf{I} \rightarrow M^{2}$ additions.

$\left(\hat{\mathbf{G}}^{*} \hat{\mathbf{G}}^{T}+\frac{\operatorname{tr}\left(\mathbf{C}_{\mathbf{w}}\right)}{E_{t r}} \mathbf{I}\right)^{-1} \rightarrow \frac{M^{2}+M}{2}$ divisions

$+\frac{2 M^{3}+3 M^{2}-5 M}{6}$ multiplications $+\frac{2 M^{3}+3 M^{2}-5 M}{6}$ additions.

$\left(\hat{\mathbf{G}}^{*} \hat{\mathbf{G}}^{T}+\frac{\operatorname{tr}\left(\mathbf{C}_{\mathbf{w}}\right)}{E_{t r}} \mathbf{I}\right)^{-1} \hat{\mathbf{G}}^{*} \rightarrow M^{2} K$ multiplications

$+M K(M-1)$ additions.

$\mathbf{N}^{-1} \rightarrow \frac{K^{2}+K}{2}$ divisions $+\frac{2 K^{3}+3 K^{2}-5 K}{6}$ multiplications

$+\frac{2 K^{3}+3 K^{2}-5 K}{6}$ additions.

$\left(\hat{\mathbf{G}}^{*} \hat{\mathbf{G}}^{T}+\frac{\operatorname{tr}\left(\mathbf{C}_{\mathbf{w}}\right)}{E_{t r}} \mathbf{I}\right)^{-1} \hat{\mathbf{G}}^{*} \mathbf{N}^{-1} \rightarrow M K^{2}$ multiplications

$+M K(K-1)$ additions.

$\frac{f_{\mathrm{MMSE}}}{\sqrt{\rho_{f}}}\left(\hat{\mathbf{G}}^{*} \hat{\mathbf{G}}^{T}+\frac{\operatorname{tr}\left(\mathbf{C}_{\mathbf{w}}\right)}{E_{t r}} \mathbf{I}\right)^{-1} \hat{\mathbf{G}}^{*} \mathbf{N}^{-1} \rightarrow 1$ division $+M K$ multiplications

+1 square root 
Overall Complexity:

$$
\begin{aligned}
& \text { Divisions }=\frac{M^{2}+M}{2}+\frac{K^{2}+K}{2}+3 \\
& \text { Multiplications }=2 M K^{2}+3 M^{2} K+M^{2}+\frac{2 M^{3}+3 M^{2}-5 M}{6} \\
& +\frac{2 K^{3}+3 K^{2}-5 K}{6}+M K \\
& =\frac{M^{3}}{3}+M^{2}\left(3 K+\frac{3}{2}\right)+M\left(2 K^{2}-\frac{5}{6}+K\right)+\frac{2 K^{3}+3 K^{2}-5 K}{6} \\
& \text { Additions }=2 M K(K-1)+2 M^{2}(K-1)+(M-1)+(K-1)+M^{2} \\
& +\frac{2 M^{3}+3 M^{2}-5 M}{6}+M K(M-1)+\frac{2 K^{3}+3 K^{2}-5 K}{6} \\
& =\frac{M^{3}}{3}+M^{2}(3 K-1 / 2)+M\left(2 K^{2}-3 K+\frac{1}{6}\right)+\frac{2 K^{3}+3 K^{2}+K}{6}-2
\end{aligned}
$$

Square Roots $=2$

According to the Big O notation, the complexity of the MMSE precoder is $\mathcal{O}\left(M^{3}\right)$.

\section{RMMSE Precoder}

$$
\begin{aligned}
\mathbf{P}_{\mathrm{RMMSE}}= & \frac{f_{\mathrm{RMMSE}}}{\sqrt{\rho_{f}}}\left(\hat{\mathbf{G}}^{*} \hat{\mathbf{G}}^{T}+\frac{\operatorname{tr}\left(\mathbf{C}_{w}\right)}{E_{t r}} \mathbf{I}_{M}\right. \\
& \left.+\frac{\Gamma f_{\mathrm{RMMSE}}^{2}\left(E_{t r} \mathbf{M}-f_{\mathrm{RMMSE}}^{2} \operatorname{tr}\left(\mathbf{M} \tilde{\mathbf{P}} \mathbf{C}_{s} \tilde{\mathbf{P}}^{H}\right) \mathbf{I}_{M}\right)}{\rho_{f} E_{t r}}\right)^{-1} \hat{\mathbf{G}}^{*} \mathbf{N}^{-1}
\end{aligned}
$$

with

$$
f_{\text {RMMSE }}=\sqrt{\frac{E_{t r}}{\operatorname{tr}\left(\tilde{\mathbf{P}} \mathbf{C}_{\mathbf{s}} \tilde{\mathbf{P}}^{H}\right)}} .
$$

For the RMMSE precoder, the computational complexity will be almost 
the same of the MMSE precoder, but with an additional cost as shown below:

$\mathbf{M} \tilde{\mathbf{P}} \rightarrow M^{2} K$ multiplications $+M K(M-1)$ additions

$\mathbf{M} \tilde{\mathbf{P}} \mathbf{C}_{s} \rightarrow M K^{2}$ multiplications $+M K(K-1)$ additions

$\mathbf{M} \tilde{\mathbf{P}} \mathbf{C}_{s} \tilde{\mathbf{P}}^{H} \rightarrow M^{2} K$ multiplications $+M^{2}(K-1)$ additions

$\operatorname{tr}\left(\mathbf{M} \tilde{\mathbf{P}} \mathbf{C}_{s} \tilde{\mathbf{P}}^{H}\right) \rightarrow(M-1)$ additions

$f_{\mathrm{RMMSE}}^{2} \operatorname{tr}\left(\mathbf{M} \tilde{\mathbf{P}} \mathbf{C}_{s} \tilde{\mathbf{P}}^{H}\right) \mathbf{I}_{M} \rightarrow\left(M^{2}+2\right)$ multiplications

$E_{t r} \mathbf{M} \rightarrow M^{2}$ multiplications

$E_{t r} \mathbf{M}-f_{\mathrm{RMMSE}}^{2} \operatorname{tr}\left(\mathbf{M} \tilde{\mathbf{P}} \mathbf{C}_{s} \tilde{\mathbf{P}}^{H}\right) \mathbf{I}_{M} \rightarrow M^{2}$ additions

$\frac{\Gamma f_{\mathrm{RMMSE}}^{2}}{\rho_{f} E_{t r}} \rightarrow 1$ divisions +2 multiplications

$\frac{\Gamma f_{\mathrm{RMMSE}}^{2}\left(E_{t r} \mathbf{M}-f_{\mathrm{RMMSE}}^{2} \operatorname{tr}\left(\mathbf{M} \tilde{\mathbf{P}} \mathbf{C}_{s} \tilde{\mathbf{P}}^{H}\right) \mathbf{I}_{M}\right)}{\rho_{f} E_{t r}} \rightarrow M^{2}$ multiplications

$\frac{\operatorname{tr}\left(\mathbf{C}_{\mathbf{w}}\right)}{E_{t r}} \mathbf{I}_{M}+\frac{\Gamma f_{\mathrm{RMMSE}}^{2}\left(E_{t r} \mathbf{M}-f_{\mathrm{RMMSE}}^{2} \operatorname{tr}\left(\mathbf{M} \tilde{\mathbf{P}} \mathbf{C}_{s} \tilde{\mathbf{P}}^{H}\right) \mathbf{I}_{M}\right)}{\rho_{f} E_{t r}} \rightarrow M^{2}$ additions

Overall Complexity:

Divisions $=\frac{M^{2}+M}{2}+\frac{K^{2}+K}{2}+4$

Multiplications $=3 M K^{2}+5 M^{2} K+4 M^{2}+\frac{2 M^{3}+3 M^{2}-5 M}{6}$

$+\frac{2 K^{3}+3 K^{2}-5 K}{6}+M K+4$

$=\frac{M^{3}}{3}+M^{2}\left(5 K+\frac{9}{2}\right)+M\left(3 K^{2}-\frac{5}{6}+K\right)+\frac{2 K^{3}+3 K^{2}-5 K}{6}+4$

Additions $=3 M K(K-1)+3 M^{2}(K-1)+2(M-1)+(K-1)+3 M^{2}$

$+\frac{2 M^{3}+3 M^{2}-5 M}{6}+2 M K(M-1)+\frac{2 K^{3}+3 K^{2}-5 K}{6}$

$=\frac{M^{3}}{3}+M^{2}(5 K+1 / 2)+M\left(3 K^{2}-5 K+\frac{11}{6}\right)+\frac{2 K^{3}+3 K^{2}+K}{6}-3$

Square Roots $=2$

According to the Big O notation, the complexity of the RMMSE precoder is $\mathcal{O}\left(M^{3}\right)$.

\section{ZF Precoder}

$$
\mathbf{P}_{\mathrm{ZF}}=\left(\hat{\mathbf{G}}^{*} \hat{\mathbf{G}}^{T}\right)^{-1} \hat{\mathbf{G}}^{*}
$$




$$
\begin{aligned}
& \hat{\mathbf{G}}^{*} \hat{\mathbf{G}}^{T} \rightarrow M^{2} K \text { multiplications }+M^{2}(K-1) \text { additions. } \\
& \left(\hat{\mathbf{G}}^{*} \hat{\mathbf{G}}^{T}\right)^{-1} \rightarrow \frac{M^{2}+M}{2} \text { divisions }+\frac{2 M^{3}+3 M^{2}-5 M}{6} \text { multiplications } \\
& \quad+\frac{2 M^{3}+3 M^{2}-5 M}{6} \text { additions. } \\
& \left(\hat{\mathbf{G}}^{*} \hat{\mathbf{G}}^{T}\right)^{-1} \hat{\mathbf{G}}^{*} \rightarrow M^{2} K \text { multiplications }+M K(M-1) \text { additions. }
\end{aligned}
$$

Overall Complexity:

$$
\begin{aligned}
& \text { Divisions }=\frac{M^{2}+M}{2} \\
& \text { Multiplications }=2 M^{2} K+\frac{2 M^{3}+3 M^{2}-5 M}{6} \\
& \qquad=\frac{M^{3}}{3}+M^{2}\left(2 K+\frac{1}{2}\right)-\frac{5 M}{6} \\
& \text { Additions }=M^{2}(K-1)+\frac{2 M^{3}+3 M^{2}-5 M}{6}+M K(M-1) \\
& \qquad=\frac{M^{3}}{3}+M^{2}\left(2 K-\frac{1}{2}\right)-M\left(\frac{5}{6}+K\right)
\end{aligned}
$$

According to the Big O notation, the complexity of the ZF precoder is $\mathcal{O}\left(M^{3}\right)$.

If the matrix inversion lemma, [63], is applied to the MMSE, RMMSE and ZF precoders, the complexity can decrease from $\mathcal{O}\left(M^{3}\right)$ to $\mathcal{O}\left(K^{3}\right)$.

\section{CB Precoder}

There is no complexity involved in calculating the CB precoder since it is just

$$
\mathbf{P}_{\mathrm{CB}}=\hat{\mathbf{G}}^{*}
$$

\section{A.3 \\ Precoders + Power Allocation}

\section{MMSE Precoder + Power Allocation}

$$
\begin{aligned}
\mathbf{P}_{\mathrm{MMSE}} \mathbf{N}_{\mathrm{MMSE}} \rightarrow & M K^{2} \text { multiplications }+M K(K-1) \text { additions } \\
& +K \text { square roots. }
\end{aligned}
$$




\section{RMMSE Precoder + Power Allocation}

$$
\begin{aligned}
\mathbf{P}_{\text {RMMSE }} \mathbf{N}_{\text {RMMSE }} \rightarrow & M K^{2} \text { multiplications }+M K(K-1) \text { additions } \\
& +K \text { square roots. }
\end{aligned}
$$

\section{ZF Precoder + Power Allocation}

$$
\begin{aligned}
\mathbf{P}_{\mathrm{ZF}} \mathbf{N}_{\mathrm{ZF}} \rightarrow & M K^{2} \text { multiplications }+M K(K-1) \text { additions } \\
& +K \text { square roots. }
\end{aligned}
$$

\section{CB Precoder + Power Allocation}

$\mathbf{P}_{\mathrm{CB}} \odot \mathbf{N}_{\mathrm{CB}}=\hat{\mathbf{G}}^{*} \odot \mathbf{N}_{\mathrm{CB}} \rightarrow M K$ multiplications $+M K$ square roots. (A-10)

\section{A. 4 \\ SINR Computation}

MMSE and RMMSE Precoders from (3-47) or (4-37)

$$
\begin{aligned}
& \operatorname{SINR}_{k, \mathrm{MMSE}}=\frac{\rho_{f} \eta_{k} \psi_{k}}{\sigma_{w}^{2}+\rho_{f} \sum_{i=1, i \neq k}^{K} \eta_{i} \phi_{k, i}+\rho_{f} \sum_{i=1}^{K} \eta_{i} \gamma_{k, i}}, \\
& \psi_{k}=\mathbb{E}\left[\mathbf{p}_{k}^{H} \hat{\mathbf{g}}_{k}^{*} \hat{\mathbf{g}}_{k}^{T} \mathbf{p}_{k}\right] \\
& \hat{\mathbf{g}}_{k}^{*} \hat{\mathbf{g}}_{k}^{T} \rightarrow M^{2} \text { multiplications. } \\
& \mathbf{p}_{k}^{H} \hat{\mathbf{g}}_{k}^{*} \hat{\mathbf{g}}_{k}^{T} \rightarrow M^{2} \text { multiplications }+M(M-1) \text { additions. } \\
& \mathbf{p}_{k}^{H} \hat{\mathbf{g}}_{k}^{*} \hat{\mathbf{g}}_{k}^{T} \mathbf{p}_{k} \rightarrow M \text { multiplications }+(M-1) \text { additions. } \\
& \text { Multiplications }=2 M^{2}+M . \\
& \text { Additions }=(M-1)(M+1) \\
& \quad=M^{2}-1 .
\end{aligned}
$$


$\phi_{k, i}=\mathbb{E}\left[\mathbf{p}_{i}^{H} \hat{\mathbf{g}}_{k}^{*} \hat{\mathbf{g}}_{k}^{T} \mathbf{p}_{i}\right], i \neq k, i=1, \ldots, K$.

$\hat{\mathbf{g}}_{k}^{*} \hat{\mathbf{g}}_{k}^{T} \rightarrow M^{2}$ multiplications.

$\mathbf{p}_{i}^{H} \hat{\mathbf{g}}_{k}^{*} \hat{\mathbf{g}}_{k}^{T} \rightarrow M^{2}(K-1)$ multiplications $+M(M-1)(K-1)$ additions.

$\mathbf{p}_{i}^{H} \hat{\mathbf{g}}_{k}^{*} \hat{\mathbf{g}}_{k}^{T} \mathbf{p}_{i} \rightarrow M(K-1)$ multiplications $+(M-1)(K-1)$ additions.

Multiplications $=M^{2}+M^{2}(K-1)+M(K-1)$

$$
=M^{2} K+M(K-1)
$$

Additions $=M(M-1)(K-1)+(M-1)(K-1)$

$$
=M^{2}(K-1)-K+1
$$

$$
\begin{aligned}
& \boldsymbol{\gamma}_{k}=\operatorname{diag}\left\{\mathbb{E}\left[\mathbf{P}_{\mathrm{MMSE}}^{H} \mathbb{E}\left[\tilde{\mathbf{g}}_{k}^{*} \tilde{\mathbf{g}}_{k}^{T}\right] \mathbf{P}_{\mathrm{MMSE}}\right]\right\} \\
& \hat{\mathbf{g}}_{k}^{*} \hat{\mathbf{g}}_{k}^{T} \rightarrow M^{2} \text { multiplications. } \\
& \mathbf{P}_{\mathrm{MMSE}}^{H} \mathbb{E}\left[\tilde{\mathbf{g}}_{k}^{*} \tilde{\mathbf{g}}_{k}^{T}\right] \rightarrow M^{2} K \text { multiplications }+M K(M-1) \text { additions. } \\
& \mathbf{P}_{\mathrm{MMSE}}^{H} \mathbb{E}\left[\tilde{\mathbf{g}}_{k}^{*} \tilde{\mathbf{g}}_{k}^{T}\right] \mathbf{P}_{\mathrm{MMSE}} \rightarrow M K^{2} \text { multiplications }+K^{2}(M-1) \text { additions. } \\
& \text { Multiplications }=M^{2}+M^{2} K+M K^{2} \\
& \quad=M^{2}(K+1)+M K^{2} \\
& \text { Additions }=M K(M-1)+K^{2}(M-1) \\
& \quad=M^{2} K+M\left(K^{2}-K\right)-K^{2}
\end{aligned}
$$

$$
\operatorname{SINR}_{k, \text { MMSE }}=\frac{\rho_{f} \eta_{k} \psi_{k}}{\sigma_{w}^{2}+\rho_{f} \sum_{i=1, i \neq k}^{K} \eta_{i} \phi_{k, i}+\rho_{f} \sum_{i=1}^{K} \eta_{i} \gamma_{k, i}}
$$

$\rho_{f} \eta_{k} \psi_{k} \rightarrow 2$ multiplications.

$\rho_{f} \sum_{i=1, i \neq k}^{K} \eta_{i} \phi_{k, i} \rightarrow K$ multiplications $+K-2$ additions.

$\rho_{f} \sum_{i=1}^{K} \eta_{i} \gamma_{k, i} \rightarrow K+1$ multiplications $+K-1$ additions.

$\sigma_{w}^{2}+\rho_{f} \sum_{i=1, i \neq k}^{K} \eta_{i} \phi_{k, i}+\rho_{f} \sum_{i=1}^{K} \eta_{i} \gamma_{k, i} \rightarrow 2$ additions.

Divisions $=1$

Multiplications $=2 K+3$

Additions $=2 K-1$

Overall Complexity: 
Divisions $=1$

Multiplications $=2 M^{2}+M+M^{2} K+M(K-1)+M^{2}(K+1)$

$+M K^{2}+2 K+3$

$=M^{2}(2 K+3)+M\left(K^{2}+K\right)+2 K+3$

Additions $=M^{2}-1+M^{2}(K-1)-K+1+M^{2} K+M\left(K^{2}-K\right)-K^{2}+2 K-1$ $=M^{2}(2 K)+M\left(K^{2}-K\right)-K^{2}+K+1$

Given that $\mathrm{SINR}_{k, \mathrm{MMSE}}$, for $k=1, \ldots, K$, the complexity of the SINR computation for the MMSE precoder is $\mathcal{O}\left(M^{2} K^{2}\right)$.

\section{ZF Precoder}

$$
\mathrm{SINR}_{k, \mathrm{ZF}}=\frac{\rho_{f} \eta_{k}}{\sigma_{w}^{2}+\rho_{f} \sum_{i=1}^{K} \eta_{i} \gamma_{k, i}}
$$

$\gamma_{k}=\operatorname{diag}\left\{\mathbb{E}\left[\mathbf{P}_{\mathrm{ZF}}^{H} \mathbb{E}\left[\tilde{\mathbf{g}}_{k}^{*} \tilde{\mathbf{g}}_{k}^{T}\right] \mathbf{P}_{\mathrm{ZF}}\right]\right\}$

$\mathbb{E}\left[\tilde{\mathbf{g}}_{k}^{*} \tilde{\mathbf{g}}_{k}^{T}\right] \rightarrow M^{2}$ multiplications.

$\mathbf{P}_{\mathrm{ZF}}^{H} \mathbb{E}\left[\tilde{\mathbf{g}}_{k}^{*} \tilde{\mathbf{g}}_{k}^{T}\right] \rightarrow M^{2} K$ multiplications $+M K(M-1)$ additions.

$\mathbf{P}_{\mathrm{ZF}}^{H} \mathbb{E}\left[\tilde{\mathbf{g}}_{k}^{*} \tilde{\mathbf{g}}_{k}^{T}\right] \mathbf{P}_{\mathrm{ZF}} \rightarrow M K^{2}$ multiplications $+K^{2}(M-1)$ additions.

Multiplications $=M^{2}+M^{2} K+M K^{2}$

$$
=M^{2}(K+1)+M K^{2}
$$

Additions $=M K(M-1)+K^{2}(M-1)$

$$
=M^{2} K+M\left(K^{2}-K\right)-K^{2}
$$

$\mathrm{SINR}_{k, \mathrm{ZF}}=\frac{\rho_{f} \eta_{k}}{\sigma_{w}^{2}+\rho_{f} \sum_{i=1}^{K} \eta_{i} \gamma_{k, i}}$

$\rho_{f} \eta_{k} \rightarrow 1$ multiplications.

$\rho_{f} \sum_{i=1}^{K} \eta_{i} \gamma_{k, i} \rightarrow K+1$ multiplications $+K-1$ additions.

$\sigma_{w}^{2}+\rho_{f} \sum_{i=1}^{K} \eta_{i} \gamma_{k, i} \rightarrow 1$ additions.

Divisions $=1$

Multiplications $=K+2$

Additions $=K$ 
Overall Complexity:

$$
\begin{aligned}
& \text { Divisions }=1 \\
& \text { Multiplications }=M^{2}(K+1)+M K^{2}+K+2 \\
& \text { Additions }=M^{2} K+M\left(K^{2}-K\right)-K^{2}+K
\end{aligned}
$$

Given that $\operatorname{SINR}_{k, \mathrm{ZF}}$, for $k=1, \ldots, K$, the complexity of the SINR computation for the ZF precoder is $\mathcal{O}\left(M^{2} K^{2}\right)$.

\section{CB Precoder}

$$
\operatorname{SINR}_{k, \mathrm{CB}}=\frac{\rho_{f}\left(\sum_{m=1}^{M} \sqrt{\eta_{m k}} \alpha_{m k}\right)^{2}}{\sigma_{w}^{2}+\rho_{f} \sum_{i=1}^{K} \sum_{m=1}^{M} \eta_{m i} \beta_{m k} \alpha_{m i}},
$$

$\sum_{m=1}^{M} \sqrt{\eta_{m k}} \alpha_{m k} \rightarrow M$ multiplications $+(M-1)$ additions $+M$ square roots.

$\rho_{f}\left(\sum_{m=1}^{M} \sqrt{\eta_{m k}} \alpha_{m k}\right)^{2} \rightarrow 2$ multiplications.

$\sigma_{w}^{2}+\rho_{f} \sum_{i=1}^{K} \sum_{m=1}^{M} \eta_{m i} \beta_{m k} \alpha_{m i} \rightarrow(2 M K+1)$ multiplications

$$
+(M-1)(K-1)+1 \text { additions. }
$$

Overall Complexity:

$$
\begin{aligned}
& \text { Divisions }=1 \\
& \text { Multiplications }=M+2+2 M K+1 \\
& \quad=M(2 K+1)+3 \\
& \text { Additions }=M-1+(M-1)(K-1)+1 \\
& \quad=M K-K+1
\end{aligned}
$$

Square Roots $=M$

Given that $\mathrm{SINR}_{k, \mathrm{CB}}$, for $k=1, \ldots, K$, the complexity of the SINR computation for the $\mathrm{CB}$ precoder is $\mathcal{O}\left(M K^{2}\right)$.

\section{A.5}

Power Allocation

\section{OPA Algorithm}




\section{A.5.0.0}

\section{MMSE and RMMSE Precoders}

$$
\left(\rho_{f} \eta_{k} \psi_{k}\right)-t\left(\sigma_{w}^{2}+\rho_{f} \sum_{i=1, i \neq k}^{K} \eta_{i} \phi_{k, i}+\rho_{f} \sum_{i=1}^{K} \eta_{i} \gamma_{k, i}\right), \text { for } t=1, \ldots, T_{\mathrm{OPA}}
$$

$\rho_{f} \eta_{k} \psi_{k} \rightarrow 2$ multiplications.

$\rho_{f} \sum_{i=1, i \neq k}^{K} \eta_{i} \phi_{k, i} \rightarrow K$ multiplications $+(K-2)$ additions.

$\rho_{f} \sum_{i=1}^{K} \eta_{i} \gamma_{k, i} \rightarrow(K+1)$ multiplications $+(K-1)$ additions.

$t\left(\sigma_{w}^{2}+\rho_{f} \sum_{i=1, i \neq k}^{K} \eta_{i} \phi_{k, i}+\rho_{f} \sum_{i=1}^{K} \eta_{i} \gamma_{k, i}\right) \rightarrow 1$ multiplication +2 additions.

$\left(\rho_{f} \eta_{k} \psi_{k}\right)-t\left(\sigma_{w}^{2}+\rho_{f} \sum_{i=1, i \neq k}^{K} \eta_{i} \phi_{k, i}+\rho_{f} \sum_{i=1}^{K} \eta_{i} \gamma_{k, i}\right) \rightarrow 1$ addition.

$\sum_{i=1}^{K} \eta_{i} \delta_{m i} \rightarrow K$ multiplications $+(K-1)$ additions.

Multiplications $=T_{\mathrm{OPA}}(K(2+K+K+1+1)+M K)$

$$
=T_{\mathrm{OPA}}\left(M K+2 K^{2}+4 K\right)
$$

Additions $=T_{\mathrm{OPA}}(K(K-2+K-1+2+1)+M(K-1))$

$$
=T_{\mathrm{OPA}}\left(M K-M+2 K^{2}\right)
$$

An SDPT3 solver is applied to compute the OPA algorithm. To solve this problem, it may require $\mathcal{O}\left(T_{\mathrm{OPA}} K^{3.5}\right)$ [64].

Since usually $K^{3.5}>M K$, the complexity of the OPA algorithm for the MMSE and RMMSE precoders is $\mathcal{O}\left(T_{\mathrm{OPA}} K^{3.5}\right)$. 


\section{A.5.0.0}

\section{ZF Precoder}

$\left(\rho_{f} \eta_{k}\right)-t\left(\sigma_{w}^{2}+\rho_{f} \sum_{i=1}^{K} \eta_{i} \gamma_{k, i}\right)$, for $t=1, \ldots, T_{\mathrm{OPA}}$

$\rho_{f} \eta_{k} \rightarrow 1$ multiplication.

$\rho_{f} \sum_{i=1}^{K} \eta_{i} \gamma_{k, i} \rightarrow(K+1)$ multiplications $+(K-1)$ additions.

$t\left(\sigma_{w}^{2}+\rho_{f} \sum_{i=1}^{K} \eta_{i} \gamma_{k, i}\right) \rightarrow 1$ multiplication +1 addition.

$\left(\rho_{f} \eta_{k}\right)-t\left(\sigma_{w}^{2}+\rho_{f} \sum_{i=1}^{K} \eta_{i} \gamma_{k, i}\right) \rightarrow 1$ addition.

$\sum_{i=1}^{K} \eta_{i} \delta_{m i} \rightarrow K$ multiplications $+(K-1)$ additions.

Multiplications $=T_{\mathrm{OPA}}(K(1+K+1+1)+M K)$

$$
=T_{\mathrm{OPA}}\left(M K+K^{2}+3 K\right)
$$

Additions $=T_{\mathrm{OPA}}(K(K-1+1+1)+M(K-1))$

$$
=T_{\mathrm{OPA}}\left(M K-M+K^{2}+K\right)
$$

As in the OPA algorithm for MMSE and RMMSE precoders, the complexity is also $\mathcal{O}\left(T_{\mathrm{OPA}} K^{3.5}\right)$, [64], considering that $K^{3.5}>M K$. 


\section{A.5.0.0}

\section{CB Precoder}

$\rho_{f} \boldsymbol{\beta}_{k} \rightarrow M$ multiplications.

$\rho_{f} \boldsymbol{\beta}_{k} \boldsymbol{\alpha}_{i}$ for $i=1, \ldots, K, \rightarrow M K$ multiplications.

$\sqrt{\rho_{f} \boldsymbol{\beta}_{k} \boldsymbol{\alpha}_{i}}$ for $i=1, \ldots, K, \rightarrow M K$ square roots.

$A_{i} x \rightarrow(M K+1)^{2}$ multiplications $+M K(M K+1)$ additions.

$A_{i} x+b_{i} \rightarrow M K+1$ additions.

$\left\|A_{i} x+b_{i}\right\|_{2} \rightarrow(M K+1)$ multiplications $+M K$ additions.

$c_{i}^{T} x \rightarrow(M K+1)$ multiplications $+M K$ additions.

$\sum_{i=1}^{K} \eta_{i} \delta_{m i} \rightarrow K$ multiplications $+(K-1)$ additions.

Multiplications $=T_{\mathrm{OPA}}\left(K\left(M+M K+(M K+1)^{2}+(M K+1)+(M K+1)\right)+M K\right)$

$$
=T_{\mathrm{OPA}}\left(M^{2} K^{3}+5 M K^{2}+2 M K+3\right)
$$

Additions $=T_{\mathrm{OPA}}(K(M K(M K+1)+M K+1+M K+M K)+M(K-1))$

$$
=T_{\mathrm{OPA}}\left(M^{2} K^{3}+4 M K^{2}+M K-M+K\right)
$$

Square Roots $=T_{\mathrm{OPA}}(M K)$

Again, the SDPT3 solver is used to perform the OPA algorithm for the CB precoder. However, differently from previous precoders, the complexity of this algorithm is higher since $M K$ variables are being calculated. Therefore, the complexity is $\mathcal{O}\left(T_{\mathrm{OPA}}(M K)^{3.5}\right)$, [64].

\section{APA Algorithm}

$$
\mathbf{N}[i+1]=\mathbf{N}[i]-\mu\left(-f^{-1} \sqrt{\rho_{f}} \mathbf{P}^{H} \hat{\mathbf{G}}^{*} \mathbf{C}_{\mathbf{s}}+f^{-2} \rho_{f} \mathbf{P}^{H} \hat{\mathbf{G}}^{*} \hat{\mathbf{G}}^{T} \mathbf{P} \mathbf{N}[i] \mathbf{C}_{\mathbf{s}}\right)
$$


$\mathbf{P}^{H} \hat{\mathbf{G}}^{*} \rightarrow M K^{2}$ multiplications $+K^{2}(M-1)$ additions.

$\mathbf{P}^{H} \hat{\mathbf{G}}^{*} \mathbf{C}_{\mathbf{s}} \rightarrow K^{3}$ multiplications $+K^{2}(K-1)$ additions.

$f^{-1} \sqrt{\rho_{f}} \rightarrow 1$ division +1 square root.

$f^{-1} \sqrt{\rho_{f}} \mathbf{P}^{H} \hat{\mathbf{G}}^{*} \mathbf{C}_{\mathbf{s}} \rightarrow K^{2}$ multiplications.

$\mathbf{P}^{H} \hat{\mathbf{G}}^{*} \hat{\mathbf{G}}^{T} \rightarrow M K^{2}$ multiplications $+M K(K-1)$ additions.

$\mathbf{P}^{H} \hat{\mathbf{G}}^{*} \hat{\mathbf{G}}^{T} \mathbf{P} \rightarrow M K^{2}$ multiplications $+K^{2}(M-1)$ additions.

$\mathbf{P}^{H} \hat{\mathbf{G}}^{*} \hat{\mathbf{G}}^{T} \mathbf{P} \mathbf{N}[i] \rightarrow K^{3}$ multiplication $+K^{2}(K-1)$ additions.

$\mathbf{P}^{H} \hat{\mathbf{G}}^{*} \hat{\mathbf{G}}^{T} \mathbf{P} \mathbf{N}[i] \mathbf{C}_{\mathbf{s}} \rightarrow K^{3}$ multiplications $+K^{2}(K-1)$ additions.

$f^{-2} \rho_{f} \rightarrow 1$ division +1 multiplication.

$f^{-2} \rho_{f} \mathbf{P}^{H} \hat{\mathbf{G}}^{*} \hat{\mathbf{G}}^{T} \mathbf{P} \mathbf{N}[i] \mathbf{C}_{\mathbf{s}} \rightarrow K^{2}$ multiplications.

$-f^{-1} \sqrt{\rho_{f}} \mathbf{P}^{H} \hat{\mathbf{G}}^{*} \mathbf{C}_{\mathbf{s}}+f^{-2} \rho_{f} \mathbf{P}^{H} \hat{\mathbf{G}}^{*} \hat{\mathbf{G}}^{T} \mathbf{P} \mathbf{N}[i] \mathbf{C}_{\mathbf{s}} \rightarrow K^{2}$ additions.

$\mathbf{N}[i]-\mu\left(-f^{-1} \sqrt{\rho_{f}} \mathbf{P}^{H} \hat{\mathbf{G}}^{*} \mathbf{C}_{\mathbf{s}}+f^{-2} \rho_{f} \mathbf{P}^{H} \hat{\mathbf{G}}^{*} \hat{\mathbf{G}}^{T} \mathbf{P} \mathbf{N}[i] \mathbf{C}_{\mathbf{s}}\right)$

$\rightarrow K^{2}$ multiplications $+K^{2}$ additions.

Overall Complexity:

Divisions $=2$

Multiplications $=T_{\mathrm{APA}}\left(M K^{2}+K^{3}+K^{2}+M K^{2}+M K^{2}+K^{3}+K^{3}+1+K^{2}+K^{2}\right)$

$=T_{\mathrm{APA}}\left(3 M K^{2}+3 K^{3}+3 K^{2}+1\right)$

Additions $=T_{\mathrm{APA}}\left(K^{2}(M-1)+K^{2}(K-1)+M K(K-1)+K^{2}(M-1)\right.$

$\left.+K^{2}(K-1)+K^{2}(K-1)+K^{2}+K^{2}\right)$

$=T_{\mathrm{APA}}\left(M\left(3 K^{2}-K\right)+3 K^{3}-3 K^{2}\right)$

Square Roots $=T_{\mathrm{APA}}(1)$

According to the Big O notation, the complexity of the APA algorithm is $\mathcal{O}\left(T_{\mathrm{APA}} M K^{2}\right)$.

\section{UPA Algorithm}

$$
\begin{aligned}
& \eta_{k}=1 /\left(\max _{m} \sum_{i=1}^{K} \delta_{m i}\right), k=1, \ldots, K, \\
& \boldsymbol{\delta}_{m}=\operatorname{diag}\left\{\mathbb{E}\left[\mathbf{p}_{m}^{T} \mathbf{p}_{m}^{*}\right]\right\}, \text { for } m=1, \ldots, M
\end{aligned}
$$




$$
\begin{aligned}
\operatorname{diag}\left\{\mathbb{E}\left[\mathbf{p}_{m}^{T} \mathbf{p}_{m}^{*}\right]\right\} & \rightarrow M K^{2} \text { multiplications. } \\
\sum_{i=1}^{K} \delta_{m i} & \rightarrow M(K-1) \text { additions. } \\
\max _{m} \sum_{i=1}^{K} \delta_{m i} & \rightarrow M \\
1 /\left(\max _{m} \sum_{i=1}^{K} \delta_{m i}\right) & \rightarrow 1 \text { division. }
\end{aligned}
$$

Overall Complexity:

$$
\begin{aligned}
& \text { Divisions }=1 \\
& \text { Multiplications }=M K^{2} \\
& \text { Additions }=M(K-1) \\
& \text { Sorting }=M
\end{aligned}
$$

According to the Big O notation, the complexity of the UPA algorithm is $\mathcal{O}\left(M K^{2}\right)$. 